UNIVERSITE DU QUEBEC

\author{
MEHOIRE \\ PRESENTE A \\ L'UNIVERSITE DU QUEBEC A CHICOUTIMI \\ COMHE EXIGENCE PARTIELLE \\ DE LA HAITRISE EN GESTION DES PI:O
}

PAR

GILLES DERY

\begin{abstract}
CONCEPTION D'UN MODELE DE REGROUPEMENT: LES CARROSSIERS DU SAGUENAY
\end{abstract}

FEVRIER 1984 


\section{Bibliothèque}

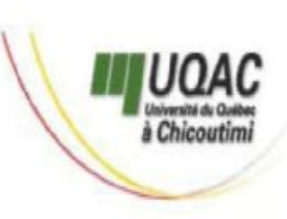

\section{Mise en garde/Advice}

Afin de rendre accessible au plus Motivated by a desire to make the grand nombre le résultat des results of its graduate students' travaux de recherche menés par ses research accessible to all, and in étudiants gradués et dans l'esprit des accordance with the rules règles qui régissent le dépôt et la governing the acceptation and diffusion des mémoires et thèses diffusion of dissertations and produits dans cette Institution, theses in this Institution, the I'Université du Québec à Université du Québec à Chicoutimi (UQAC) est fière de Chicoutimi (UQAC) is proud to rendre accessible une version make a complete version of this complète et gratuite de cette œuvre. work available at no cost to the reader.

L'auteur conserve néanmoins la The author retains ownership of the propriété du droit d'auteur qui copyright of this dissertation or protège ce mémoire ou cette thèse. thesis. Neither the dissertation or Ni le mémoire ou la thèse ni des thesis, nor substantial extracts from extraits substantiels de ceux-ci ne it, may be printed or otherwise peuvent être imprimés ou autrement reproduced without the author's reproduits sans son autorisation. permission. 
Je dédie ce rapport de Maitrise à mon épouse sylvie; mes enfants, Marie-Eve, François et Gilles-Philippe; à mes parents, Brigitte et Charles Déry; à mes beaux parents, Françoise et Joseph Bruneau. 


\section{RESUME}

Le présent mémoire a comme objectif de fournir un guide pour la réalisation d'un regroupement de services en vue de venir en aide aux petites entreprises qui éprouvent des difficultés de négociations face aux organisations oeuvrant dans leurs secteurs d'activités.

La première étape de réalisation du projet nous a permis de dégager les caractéristiques du secteur qui nous a servi de modele soit: les ateliers de débosselage du Saguenay.

Cette démarche nous a amené à découvrir une problématique commune en ce qui a trait a l'impossibilité de pouvoir établir sur une base individuelle, un rapport de force nécessaire à la survie de ces petites entreprises. Les informations recueillies, nous amènerons à élaborer un modèle de regroupement visant à éliminer ce problème. 


\begin{abstract}
La finalité de cette démarche est de pouvoir assurer une survie, voire même une croissance des entreprises en difficultés.
\end{abstract}

Supporté par un cadre méthodologique proposé
par la maîtrise, nous décrirons chaque étape de
réalisation du projet qui nous a amené à amorcer le
processus de regroupement des ateliers de débosselage.




\section{REMERCIEMENTS}

Je désire remercier mon épouse sylvie, pour sa patience exemplaire durant la réalisation de cette Maîtrise.

Mes remerciements vont également à mon
directeur de thèse, Monsieur Raymond Auger pour sa
diligence et la confiance qu'il m'a témoignée.
Je ne peux passer sous silence l'attention, la
compréhension et le support de mon directeur de
programme, Monsieur Adam Lapointe tout au cours de ces
études.

Finalement, à tous mes collègues particulièrement, Messieurs Laval Bergeron et Christian Gravel, un gros merci pour leur aide et leur encouragement. 


\section{TABLE DES MATIERES}

\section{PAGE}

CHAPITRE I - INTRODUCTION $\ldots \ldots \ldots \ldots \ldots \ldots$

1.1 - PRESENTATION DU RAPPORT............ I

1.l.1 -Premiers contacts avec le milieu 1

1.2 - OBJectifs vises............... 4

1.3 - MOYENS...................... 4

1.4 - METHODE D'INTERVEnTION .......... 5

$1.5-$ ECHEANCIER...................

$1.6-$ MANDAT $\ldots \ldots \ldots \ldots \ldots \ldots \ldots \ldots \ldots \ldots \ldots \ldots \ldots \ldots \ldots \ldots$

1.6 .1 -Approbation du sujet.........6

1.6 .2 -Mandat................

1.6.3 -Nécessité de 1 'intervention.... 7

1.6.4 -Intervenants privilégiés......7

CHAPITRE II - PROBLEMATIQUE $\ldots \ldots \ldots \ldots \ldots \ldots$

$2.1-$ INTRODUCTION.................. 
2.2 - L'ORGANISATION DE L'INDUSTRIE..........

$2.2 .1-$ La structure................

2.2 .1 .1 -organigramme...........11

2.2.2 -Description de tâches.........13

2.2 .2 .1 -Les donneurs d'ordre......13

2.2.2.2 -Les centres d'estimation....16

2.2 .2 .3 -Les ateliers de débosselage.17

2.2 .2 .4 -Le comité paritaire......18

2.2 .2 .5 -Les ateliers clandestins....18

2.2.2.6 -Les fournisseurs.........19

2.2.2.7 -Les concessionnaires......19

2.2.2.8 -Les autres organismes

gouvernementaux.........20

$2.2 .3 \quad$-Commentaires................

2.2.3.1 Les compagnies d'assurance..22

2.2.3.2 -Le centre d'estimation.....23

2.2 .3 .3 -Les estimateurs privés....27

2.2 .3 .4 -Les fournisseurs........27

$2.2 .4 \quad$ Le marché................... 30

2.2 .4 .1 -Analyse de l'ofrre et de la

demande....................

2.2 .4 .2 -La demande..............30

$2.2 .4 .3-L^{\prime}$ offre................. 34 
$2.2 .4 .4-$ La concurrence............35

2.2.4.5 -Les concessionnaires

automobiles...............

2.2 .4 .6 -Les clandestins..........37

2.2.5 -Le contexte légal............39

2.2 .5 .1 -Lois du travail........40

2.2.5.2 -Loi sur I'environnement...444

2.2 .5 .3 -Autres lois..........45

2.2.6 -Le fonctionnement de l'industrie.46

2.2.6.1 -5ystème global de

1 'industrie..........46

2.2 .7 -Les sous-systèmes...........49

2.2 .7 .1 -Sous-système octroi

de contrat...............

2.2.7.2 -Processus de réalisation

des travaux...........5 52

2.2 .7 .3 -5ous-système de gestion....54

2.2.7.4 -Commentaires..........56

2.2.8 -Les performances de l'industrie..57

2.2.9 -Le climat................59

2.3 - SELECTION DU PROBLEME A ETUdiER........60 2.3 .1 -Liste des candidats problèmes...60 
2.3.2 -Sélection et justification

du problème.............61

\section{CHAPITRE III - ANCRAGE, MODELE CONCEPTUEL ET}

COMPARAISON............63

3.1 - ANCRAGE........................63

3.1.1 -Choix du système pertinent.....663

3.1.2 -Définition du système pertinent..63

$3.1 .3 \quad-$ Validation...............64

3.2 - MOdELE CONCEPTUEL................66

3.2.1 -Les activités du systèmes

conceptuels............66

$3.2 .1 .1-0$ rientation...........68

3.2.1.2 -5tructuration..........70

3.2.1.3 -Mode de fonctionnement.....70

3.2.1.4 -Recrutement...........73

3.2.1.5 -Dpérationnalisation......75

3.2.2 -Validation du modèle conceptuel..77

\section{3 - COMPARAISON}

3.3.1 Comparaison du modèle conceptuel avec la réalité...........79 
CHAPITRE IV - IMPLANTATION............

4.1 - LES ACTIVITES................. 82

4.2 - LIGNES DIRECTRICES ET MODE DE FONCTIONNEMENT DU REGROUPEMENT DES CARROSSIERS

DU SAGUENAY INC................... 84

4.2.1 -Buts du regroupement.........84

4.2.2 -Pouvoirs de l'assemblée générale.85

4.2.3 -Rôles et pouvoirs du conseil d'administration...........85

4.2.4 -Organigramme du regroupement....87

4.2.5 -Description de tâches........87

4.2 .5 .1 -Membres.............87

4.2.5.2 -Président............90

$4.2 .5 .3-$ Vice-président.........90

$4.2 .5 .4-$ Secrétaire...........90

4.2 .5 .5 -Trésorier...........91

4.2 .5 .6 -Directeur............92

4.2.5.7 -Président de comité......92

4.2 .6 -5tatuts et règlements........992

4.2.6.1 -Conditions d'admission.....93

$4 \cdot 2.6 .2-L^{\prime}$ assemblée générale.....94

4.2.6.3 -Le conseil d'administration.96 


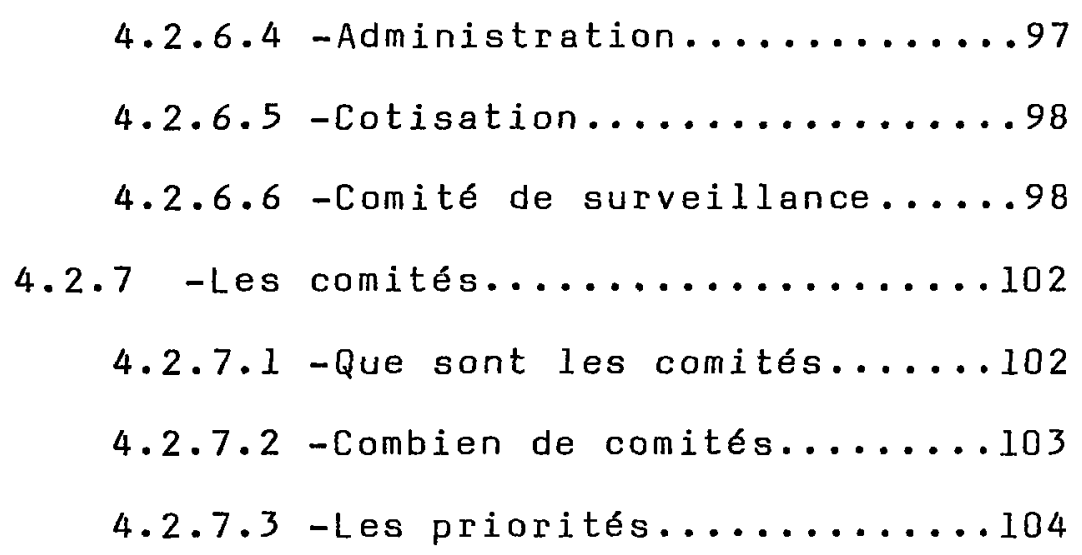

4.3 - RESUME DES ACTIVITES PAR SOUS-SYSTEMES..106 4.3.1 -Sous-système orientation......106 4.3.1.1 -Liste des activités......106 4.3.2 -5ous-système structuration.....107 4.3.2.1 -Liste des activités......109 4.3.4 -Sous-système mode de fonctionnement............111 4.3.4.1 -Liste des activités......111 4.3.5 -Sous-système recrutement......114 4.3.4.1 -Liste des activités.....115 4.3.6 -Sous-système opérationnalisationll7 4.3.6.1 -Liste des activités......117 
CHAPITRE V-CONTROLE.................

5.1 - NECESSITE DU CONTROLE..................

5.2 - LE VOLET INTERNE................... 22

5.2.1 -Le sous-système orientation....122

5.2.2 -Le sous-système structuration...123

5.2 .3 -Le sous-sytème made de

fonctionnement..............123

5.2 .4 -Le sous-système recrutement.....124

5.2 .5 -Le sous-système opération-

nalisation.................... 124

5.3 - LE VOLET EXTERnE.........................

5.3 .1 -Le décret.................125

5.3 .2 -Les ateliers clandestins......126

5.3 .3 -Les fournisseurs...........126

CHAPITRE VI - REFLEXION METHODOLOGIQUE......

6.1 - REFLEXION METHODOLOGIQUE...........128

6.1 .1 -La méthode...............128

$6.1 .2 \quad-$ La démarche...............130 
6.2 - LES CONNAISSANCES ACQUISES............. I3I 6.2.1 -Sur le contenu problématique....131

6.2 .2 -Sur les regroupements

d'entreprises................133

6.2 .3 -5ur la démarche...........136

6.2 .4 -Pour le chercheur..........138

6.3 - CONCLUSION METHODOLOGIQUE............ 140

CHAPITRE VII CONCLUSION................

7.1 Conclusion..........................

ANNEXES............................. 144

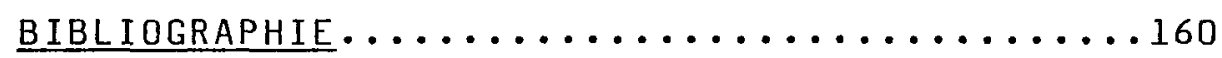




\section{LISTE DES SCHEMAS}

Page

\begin{tabular}{|c|c|c|}
\hline SCHEMA & 1 & $\begin{array}{l}\text { Organigramme de l'industrie } \\
\text { du débosselage au Saguenay......... }\end{array}$ \\
\hline SCHEMA & 2 & Le système global de l'industrie... \\
\hline SCHEMA & 3 & Le sous-système octroi de contrat.. \\
\hline SCHEMA & 4 & 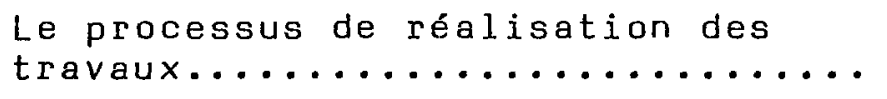 \\
\hline SCHEMA & 5 & Le sous-système de gestion........ \\
\hline SCHEMA & 6 & 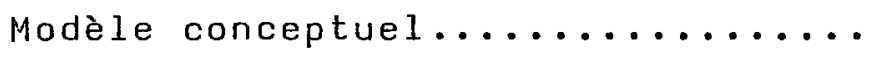 \\
\hline SCHEMA & 7 & Le sous-système orientation ....... \\
\hline SCHEMA & 8 & Le sous-système structuration..... \\
\hline SCHEMA & 9 & $\begin{array}{l}\text { Le sous-système mode de fonction- } \\
\text { nement } \ldots \ldots \ldots \ldots \ldots \ldots \ldots \ldots \ldots \ldots \ldots \ldots \ldots\end{array}$ \\
\hline SCHEMA & 10 & Le sous-système recrutement....... \\
\hline SCHEMA & 11 & Le sous-système opérationnalisation \\
\hline SCHEMA & 12 & $\begin{array}{l}\text { Organigramme du Regroupement des } \\
\text { Carrossiers du Saguenay Inc...... }\end{array}$ \\
\hline
\end{tabular}


xi i

SCHEMA 13

Rapport d'étapes des activités.....103 
xii i

\section{LISTE DES TABLEAUX}

$\underline{\text { Paqe }}$

TABLEAU 1 Frais d'exploitation des assureurs en pourcentage des primes souscrites au Québec...........26

TABLEAU 2 Evolution de l'industrie du débosselage au Saguenay............ 32 


\section{LEGENDE}

FLUX D'INFORMATION

IDENTIF ICATION DE PROBLENES

ACT IVITES

SYSTEHES ET SOUS-SYSTEMES

ENV I RONNEMENT

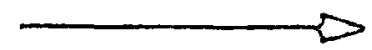

$?$
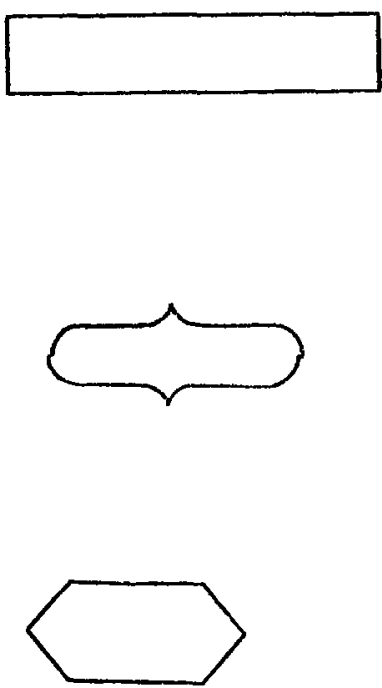
CHAP ITRE I

INTRODUCTION 


\section{CHAPITRE 1}

\section{INTRODUCTION}

\section{1 - PRESENTATION DU RAPPORT}

\section{1 .1 - Premier contact avec le milieu}

C'est en mars 1983 que j'ai été amené à m'impliquer dans le domaine de l'industrie du débosselage par l'entremise de l'Association PME et son directeur Monsieur Guy Gagné. Cette association, qui avait été mise sur pied quelques temps auparavant, visait le regroupement de toutes les PME de la région particulièrement en ce qui concerne les achats de matières premières et de services.

Dans cet optique, il avait sollicité quelques propriétaires d'ateliers de débosselage lesquels lui avaient mentionné leurs désirs de se rencontrer pour discuter de leurs problèmes communs. Devant l'intérêt manifeste de ces membres, l'ampleur de la tâche et, considérant les ressources limitées de l'Association PME, Monsieur Gagné s'adressait alors à l'Université du 
Québec à Chicoutimi au département des Sciences Economiques et Administratives afin d'obtenir la collaboration de professeurs et d'étudiants. Son dossier fut transmis au responsable du programme de la Maitrise en Gestion des Petites et Moyennes Organisations.

C'est ainsi qu'à la fin de mars 1983 , je
rencontrais ce groupe provisoire de débosseleurs afin
d'envisager la possibilité de participer à leur
démarche, pour les assister et leur permettre d'analyser
avec eux la situation de leur secteur d'activité
économique.

Par ce mémoire, je me propose donc de vaus présenter la démarche qui a été effectuée avec ce groupe de propriétaires d'ateliers de débosselage. Celle-ci a trouvé son aboutissement dans la création en juin 1983 d'une structure légale visant à regrouper tous les ateliers de débosselage du Saguenay.

Nous tenterons de démontrer la pertinence de l'association dans le contexte d'une industrie où le climat de relation entre les intervenants est perturbé, et aussi, les moyens que nous avons imaginés pour assurer sa survie. 
L'étude contiendra les éléments suivants:

- description de l'industrie du débosselage;

- présentation de la problématique propre aux ateliers de débosselage;

- mise sur pied du regroupement;

- moyens pour assurer sa survie;

- implantation et contrôle.

Le rapport fera état des activités qui ont été effectuées et surtout, la complémentarité souhaitable qui peut exister entre une institution d'enseignement tel que I'UQAC et un milieu d'affaire particulier, comme celui du débosselage.

Ceci est d'autant plus important que l'industrie avec laquelle nous avons décidé de travailler ne reçoit pas traditionnellement d'assistance de quelques sources que se soient. Ni les gouvernements, ni les autres ccrps de la société n'ont prévu, à tort ou à raison, de mécanismes en ce sens.

Qu'il nous suffise de mentionner que, dans tous les programmes d'aide mis de l'avant par les différents paliers de gouvernement, il ne s'en trouve aucun auquel peut se reférer l'industrie ou les intervenants. 


\section{$1.2-$ OBJECTIFS VISES}

Compte tenu de la démarche dans laquelle nous nous sommes engagés, un objectif est recherché initialement par la rédaction de ce mémoire. Il s'agit de doter les ateliers de débosselage de moyens leur permettant de répondre à leurs besoins propres. Cet objectif devra toutefois etre confirmé par le diagnostic.

\section{3- MOYENS}

Les moyens privilégiés dans cette démarche seront les suivants:

$$
\begin{aligned}
& \text { 1.- Par des réunions régulières avec un } \\
& \text { groupe de débosseleurs, identifier } \\
& \text { les zones problématiques; } \\
& \text { 2.- Par des recherches approfondies, } \\
& \text { apporter des alternatives } \\
& \text { de solutions adéquates; } \\
& \text { 3.- Mettre en place ces mêmes solutions; } \\
& \text { 4.- Mesurer les progrès enregistrés. }
\end{aligned}
$$




\section{4- METHODE D'INTERVENTION}

La méthode utilisée pour réaliser le mandat sera celle présentée au groupe de débosseleurs lors de notre première rencontre soit: la méthode de diagnostic de P.B. Checkland. Comme nous l'avions alors exposée, celle-ci, inspirée de la théorie des systèmes, considère l'organisation comme un ensemble constitué d'activités humaines interreliées par différents types de flux (personnel, matières premières, dollars, informations) et agencé de façon à réaliser une mission, un objectif, ou pour accomplir une fonction précise dans un super système.

Sise dans son environnement plus ou moins contrôlable, l'organisation y puise ses ressources et $y$ retourne sa production sous la.forme de produits, services ou informations.

Elle propose donc de passer d'un point de vue global vers un domaine particulier ou privilégié. Elle fut d'ailleurs conçue pour s'attaquer à des situations complexes et peu structurées c'est à dire, des situations dont il est difficile de quantifier les composantes. 


\title{
1.5- ECHEANCIER
}

\begin{abstract}
Prenant en considération les exigences accadémiques de la démarche, il est prévu cinq étapes pour sa réalisation. La première est évidemment notre proposition d'assistance au groupe de débosseleurs en mars 1983. La seconde étape, constituée de la présentaion de l'image riche, s'est terminée en juillet 1983 et la troisième, le modèle conceptuel, en novembre 1983. Le rapport est suivi périodiquement par le groupe de débosseleurs. Finalement, la démarche se concrétisera en février 1984 avec le dépôt du rapport accadémique.
\end{abstract}

\section{6- MANDAT}

\section{$1.6 .1-$ Approbation du sujet}

L'intervention étant réalisée dans le cadre de la Maîtise en Gestion des P.M.O., plus précisément à titre de mémoire, le sujet et la méthode doivent donc être l'objet d'une approbation accadémique. 


\subsection{2- Mandat}

Le mandat précisé à partir des hypothèses et de l'objectif qui en découlent doit toutefois être confirmé par le diagnostic. En effet, l'approche de P.B. Checkland prévoit qu'un point d'ancrage sera dégagé suite à L'analyse de la situation.

\subsection{3- Nécessité de l'intervention}

Il est important de prendre en considération la nécessité de l'intervention puisque l'industrie du débosselage et les débosseleurs en particulier, ont entrepris une démarche rationnelle pour tenter de solutionner leurs problèmes. Le mémoire de la Martrise représente en fait, le contenu de cette démarche et les amorces de solutions qui ont été entreprises et dont ils espèrent bénéficier.

\section{$1.6 .4-$ Intervenants privilégiés}

Compte tenu de la problématique majeure du mémoire ainsi que l'importance pratique des résultats, les atliers de débosselage constitueront les clients privilégiés à l'intérieur de ce mémoire. 
CHAP ITRE II

PROBLEMATIQUE 


\section{CHAPITRE 11}

\section{PROBLEMATIQUE}

\section{$2.1-$ INTRODUCTION}

Nous référant au code canadien de classification industrielle présentement en vigeur, les activités reliées au secteur du débosselage concerne le travail de carosserie et de peinture, ainsi que les travaux spécialisés tel que la mise au point de l'allumage, de la carburation, du parallélisme; le redressement de châssis, la remise en état de système d'échappement et la réfection des freins.

$$
\text { Quoique nous ayons tenu compte uniquement des }
$$
ateliers indépendants pour identifier notre système-client, tous les ateliers retenus effectuent directement ou par sous-traitance les activités mentionnées. Toutefois, dans le but de préciser davantage ces activités, nous incluons une liste des principaux travaux effectués chez un débosseleur. Nous avons: 
a: réparation de carosserie et remplacement de pièces;

b: application de peinture sur les véhicules;

c: redressement de châssis;

d: fabrication de pièces particulières;

e: application d'enduit antirouille;

f: travaux de soudure;

g: remplacement de pare-brise;

$h$ : travaux de mécanique.

Tous ces travaux sont offerts par tous les ateliers à des niveaux différents.

Le présent chapitre contiendra l'"image riche" du secteur du débosselage. Après avoir parlé de l'organisation de l'industrie, nous élaborerons sur le marché du débosselage, son contexte légal, son fonctionnement et sur la performance générale.

Ces étapes nous permettront alors d'identifier le climat qui prévaut dans l'industrie et la sélection du problème particulier à étudier. 


\section{2- L'organisation de I'industrie}

\section{$2.2 .1-\underline{\text { La structure }}$}

Pour comprendre la structure actuellement en vigeur dans l'industrie du débosselage au Saguenay, nous allons examiner les éléments qui sont ressortis de notre analyse. Même si, dans les faits, il n'existe pas à proprement parler de relation formelle entre les différents intervenants identifiés, il reste que ceux-ci représentent l'ensemble de ces éléments constitutifs.

Quoiqu'il puisse sembler que nous ne respectons pas en cela la méthodologie de travail que nous avons retenue au préalable, nous estimons que cela ne peut porter préjudice à la démarche globale. Nous nous référons d'ailleurs au document de Monsieur Paul Prévost qui note:

En fait, les structures, définies comme la conception d'une organisation par le truchement de laquelle l'entreprise est gérée, sont avant tout fonction des stratégies et du système d'activités humaines de l'organisation. En conséquence, l'étude d'une organisation en se centrant prioritairement sur les structures ne peut etre que limitée. c'est l'ensemble des interrelations avec 
l'environnement, les processus, les stratégies et voir, la philosophie de l'organisation qui donnent un sens aux structures. ( 1 )

La présentation se fera selon trois volets soient:

-l'organigramme de la structure;

-la description des composantes;

-les commentaires s'y rapportant.

2.2.1.1- Organigramme de la structure acuelle

Avec l'organigramme, nous vous présenterons la structure hiérarchique des intervenants de l'industíie en utilisant l'ensemble des composantes et leurs rapports respectifs. (Voir schéma No 1 )

Prévost, Paul, Le diagnostic-intervention: une approche systémique au diagnostic organisationnel et a la recherche-action, LEER, 1983, P.40. 
SCHEMA NO 1

L'INDUSTRIE DU DEBOSSELAGE AU SAGUENAY

QRGAN I GRAMME
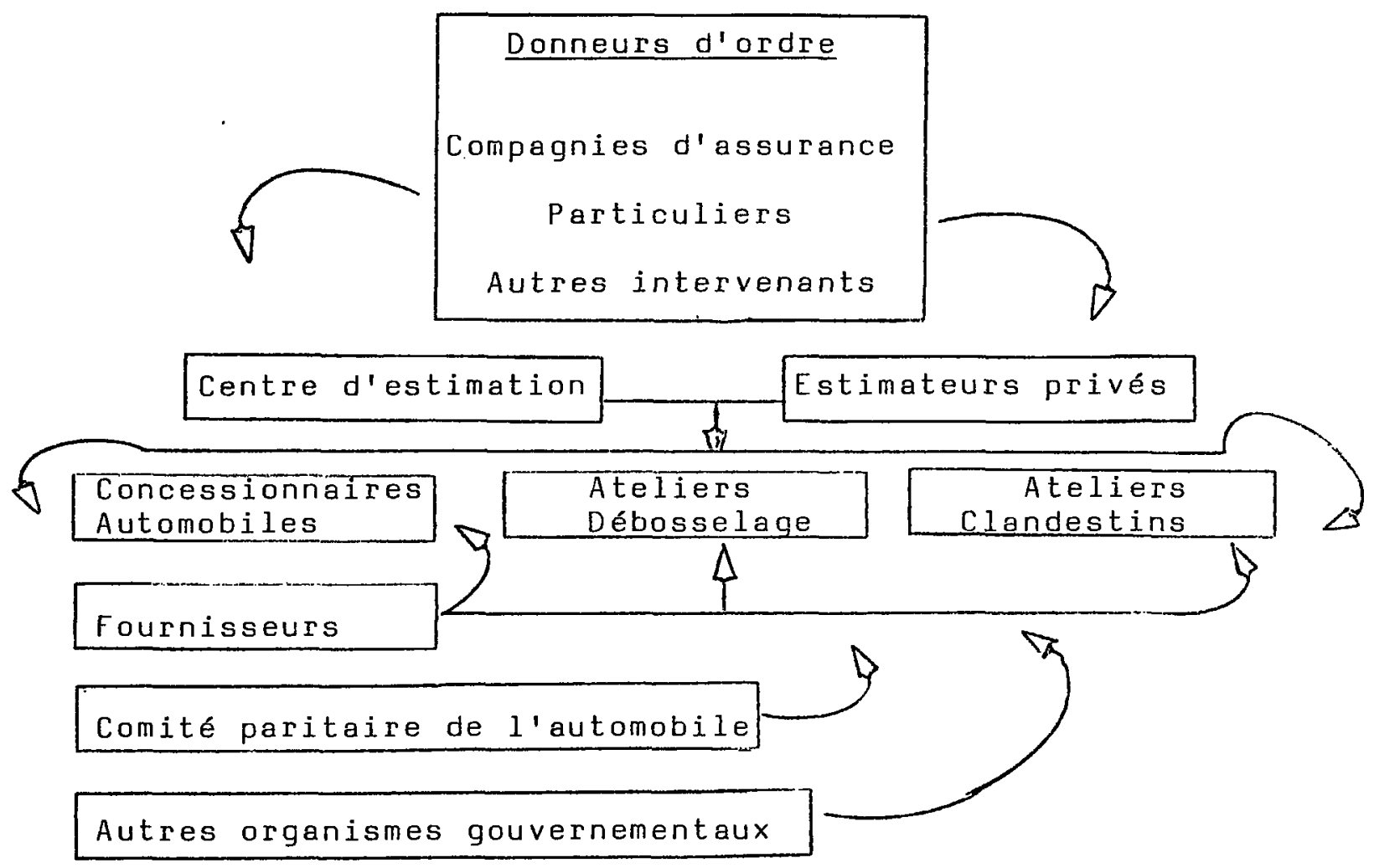


\subsection{2- Description de tâches}

Nous allons maintenant reprendre, à partir de l'organigramme, les éléments constitutifs de celui-ci.

\subsubsection{1- Les donneurs d'ordre}

Les donneurs d'ordre sont les requérants habituels de travaux dans le domaine du débosselage. Ces derniers ont été catalogués en trois types distincts:

- les compagnies d'assurance;

-les particuliers;

-les autres utilisateurs.

\section{Les compagnies d'assurance}

Formée de toutes les compagnies d'assurance habilités à oeuvrer au Québec en vertu de la Loi sur I'Assurance Automobile et du Surintendant des Assurances du Québec, cette catégorie constitue le plus important utilisateur de services pouvant être offerts par l'industrie du débosselage. D'après les 40 ateliers de débosselage que nous avons contactés dans la région, 
tous s'accordent pour dire qu'elles représentent entre 60 et $75 \%$ de tous leurs revenus annuels.

Pour l'année 1982, on comptait 137 assureurs autorisés à pratiquer et 106 ayant effectivement pratiqués. (1)

De ce nombre, 15 assureurs ou groupes d'assureurs contrôlaient $65 \%$ de tout le marché québéçois de l'assurance. (2) Quoiqu'une certaine concurrence existe dans le milieu, il reste que cette catégorie possède beaucoup d'influence et de ce fait, elle se charge de déterminer les grandes orientations du marché en particulier en ce qui concerne les structures de prix, la qualité du service et l'affectation des contrats.

I. Rapport du Surintendant des Assurances, La tarification en assurance automobile au Québec

1982.Gouvernement du Québec,1983,p.4.

2. Rapport du Surintendant des Assurances, La tarification en assurance automobile au Québec,Gouvernement du Québec,1983,P.6 


\section{Les particuliers}

Cette deuxième catégorie d'utilisateurs est beaucoup moins importante que la première et ne possède que peu d'influence sur l'industrie. Toujours d'après les ateliers que nous avons visités, cette catégorie représenterait de 15 à $25 \%$ de leurs revenus. Ces personnes vont généralement faire confiance à un atelier de débosselage particulier pour la qualité des travaux et vont respecter assez fidelement la structure de prix prescrite par l'office de Protection du Consommateur. Cependant, pour plusieurs travaux mineurs, ces personnes auront souvent recours à un atelier clandestin ou encore, effectueront eux-mêmes ces travaux.

\section{Les autres utilisateurs}

Nous voulons mentionner les autres utilisateurs plus marginaux. Nous pensons notamment aux différents paliers de gouvernement qui possèdent des flottes de véhicules et qui font effectuer, à l'occasion, des travaux non couverts par les réclamations adressées aux compagnies d'assurance. Ils procèdent par appel d'offre directement avec les ateliers de débosselage. Ceci est particulièrement vrai pour les municipalités dans la région du Saguenay. 
$2 \cdot 2 \cdot 2 \cdot 2$-Les centres d'estimation

A ce niveau, nous incluerons également les bureaux d'estimateurs privés qui effectuent la même fonction que les centres d'estimation.

Ces centres ont été crés par les compagnies d'assurance pour assurer le lien entre les assurés et les ateliers de réparations. Les principales fonctions de ce groupe sont:

- administrer les dossiers pour les assureurs;

- négocier les travaux avec les débosseleurs;

- assurer un contróle de qualité;

- réduire les couts.

Ils possèdent un pouvoir de négociation presqu'aussi important que les assureurs eux-mêmes et n'hésitent pas à l'utiliser. En fait, ils représentent le prolongement logique de la philosophie des assureurs. 


\section{2 .2 .3 - Les ateliers de débosselage}

Les ateliers de débosselage sont les exécutants des travaux consenties par les différents donneurs d'ordre. Ils sont soumis aux pressions exercées sur eux par les niveaux supérieurs de l'organigramme et, sur une base individuelle, ne possèdent que peu d'influence sur les assureurs ou les centres d'estimations. Ils sont dépendants de la concurrence favorisée par la hiérarchie et doivent respecter les nombreuses législations gouvernementales.

Ils doivent également subir la concurrence des ateliers incorporés aux concessionnaires automobiles et une autre forme, cette fois-çi déloyale, de la part des ateliers clandestins.

Jusqu'au début des présents travaux, ils ne possédaient pas de structure d'association regroupant les propriétaires d'ateliers de débosselage pouvant agir comme représentant dans le cas de problèmes les touchant et pour lesquels, une action commune serait souhaitable. 
$2.2 .2 .4-$ Le comité paritaire de l'automobile

Situé à Chicoutimi, le comité paritaire de l'industrie de l'automobile regroupe des représentants d'associations patronnales et syndicales oeuvrant dans ce domaine pour toute la région du Saguenay Lac St-Jean.

Ses principales obligations sont les suivantes:

- administrer le décret en général;

- percevoir les primes exigées;

- nommer des inspecteurs;

- enregistrer les employés et les employeurs;

- effectuer la classification des salariés;

- produire des relevés périodiques pour le ministère du travail;

- suggérer des modifications au décret.

\section{2 .2 .5 - Les ateliers clandestins}

Les ateliers clandestins sont les compétiteurs officieux des ateliers de débosselage enregistrés. Ils ne possèdent aucun statut officiel dans l'industrie. Ils sont cependant très nombreux et très actifs. D'après le 
secrétaire-gérant du comité paritaire de l'automobile, Monsieur Bruno Jean, l'estimation la plus conservatrice fait état de 2 ateliers clandestins pour 1 atelier enregistré.

Avec I'avènement du décret, ils sont, en principe, assujettis à ce dernier et doivent respecter toutes les règles en vigueur. C'est la responsabilité du comité paritaire et des inspecteurs de régulariser la situation. Toutefois, tant que ces derniers ne seront pas embauchés, il est inutile d'espérer une diminution de ces garages.

\section{$2 \cdot 2 \cdot 2 \cdot 6$-Les fournisseurs}

Cette catégorie est aussi soumise au décret en vigeur le premier juin 1983. Elle pourvoit les intéressés en matières premières et de ce fait, possède un certain pouvoir sur les ateliers de débosselage.

La situation est particulièrement visible au niveau de la fixation des prix, les fournisseurs jouant sur la situation géographique de la région et leurs nombres restreints. 


\subsubsection{7- Les concessionnaires automobiles}

Les concessionnaires jouent un double rôle dans I'industrie du débosselage. D'une part, ils sont les fournisseurs de pièces et accessoires automobiles pour les débosseleurs et d'autre part, ils compétitionnent ces mêmes personnes en effectuant également du travail de débosselage. Ils possèdent un bon pouvoir de négociation auprès du reste de la structure puisque, depuis plusieurs années, ils sont regroupés sous une même corporation et adoptent des lignes de conduite uniformes pour toute la région.

\section{2 .2 .8 - Les autres organismes gouvernementaux}

Dans cette catégorie, nous incluons tous les organismes gouvernementaux qui sont susceptibles d'avoir un impact sur l'industrie. Les principaux sont les suivants:

- C.S.S.T. ;

- Environnement Québec;

- Travail Québec;

- Revenu Québec;

- Revenu Canada. 


\section{$2 \cdot 2 \cdot 3$-Cammentaires}

Dès le début de notre intervention dans ce secteur, nous avons pu percevoir deux problèmes qui ressortaient de la structure actuelle et qui empêchaient le fonctionnement harmonieux de l'industrie.

Il y d'abord l'absence de communication entre le premier niveau de l'organigramme; les donneurs d'ordre et le second niveau, les exécutants. Cette absence est particulièrement évidente entre les assureurs et les ateliers de débosselage. Cela amène des tensions croissantes et la prise de décisions unilatérales de la part des compagnies d'assurance directement ou via les centres d'estimation.

Il y a ensuite l'absence de cohésion entre les ateliers de débosselage eux-mêmes ce qui élémine la possibilité de pouvoir négocier sur des bases communes.

Nous allons maintenant revenir d'une façon plus précise sur les activités de quatre des intervenants: les compagnies d'assurance, les centres d'estimation, les estimateurs privés et les fournisseurs. 


\section{$2.2 .3 .1-$ Les compagnies d'assurances}

Un des premiers sujets de discussion que nous avons eu avec le comité provisoire concernait justement les compagnies d'assurances et certaines pratiques d'affaire qu'elles utilisent de plus en plus. En bref, elles cherchent à réduire au minimum les réparations à apporter aux véhicules et n'hésitent pas à restreindre le temps à accorder pour ces travaux. Elles exigent la pose de pièces recyclées et cherchent par tous les moyens à couper sur le taux horaire affiché par les garages. Toutes ces démarches s'effectuent par personnes interposées lesquelles sont le Centre d'Estimation du Saguenay et/ou les ajusteurs d'assurance privés.

Cette nouvelle philosophie s'inscrit dans les politiques de restrictions mises de l'avant par les compagnies d'assurance au cours des dernières années et pour se plier aux exigences du surintendant des Assurances du Québec qui y voit un moyen de réduire les primes d'assurance des automobilistes. 


\subsubsection{2- Le Centre d'Estimation}

Le Centre d'estimation est une nouvelle structure dans le domaine du débosselage depuis 8 ans. Ces centres ont été mis sur pied par l'ensemble des compagnies d'assurance via le Bureau d'Assurance du Canada. Les buts recherchés par les compagnies étaient d'uniformiser la procédure de réclamation,d'aplanir les écarts entre les ajusteurs privés, d'assurer que le travail autorisé soit effectué adéquatement avec un meilleur contróle de la qualité, d'éliminer le favoritisme, d'accréditer les garages intéressés à effectuer des travaux et de diminuer le coot des réparations pour, ultimement, réduire les primes d' assurance automobile.

La façon de procéder, en théorie, est la suivante. Le centre accrédite les garages intéressés et leur demande de produire une fois par année le tarif qu'ils entendent facturer. Lors d'un accident, tous les véhicules impliqués doivent étre transportés au centre ou à défaut, un employé du centre doit l'examiner pour évaluer l'étendue des dommages. Une fois l'évaluation complétée,il discute celle-ci avec le débosseleur qui a 
été suggéré par le client ou encore, assigne les travaux à un atelier qu'il aura choisi et qui aura accepté le prix fixé. Si des problèmes surgissent, il visite l'atelier de débosselage et apporte, s'il y a lieu, les modifications qui s'imposent au prix initial. Finalement, il demanoe au propriétaire de se présenter avec son véhicule une fois les réparations achevées, pour l'inspection.

Dans la réalité cependant, les employés du centre tentent d'imposer les nouvelles consignes des compagnies d'assurance. Ils rognent indament sur le temps de la réparation, exigent des pièces usagées ou réusinées, coupent le tarif pourtant affiché au centre, favorisent des ateliers ou garages en particulier et ne controle que $20 \%$ des véhicules réparés. Ceci s'explique par le peu de personnes oeuvrant dans les centres destimation. Cela nous a été confirmé par le directeur du Centre d'Estimation du Saguenay Lac St-Jean.

Pour le débosseleur, il en résulte une perte de temps inutile, des déplacements exagérés et le pire, dans bien des cas, un retard dans l'achevement de travaux avec les pertes de revenus qui s'en suivent. 
Malgré toutes ces démarches, il ne semble pas que nous assistions à des réductions sensibles des primes d'assurances, celles-ci étant plutôt fonction des facteurs suivants:

- le territoire de l'assuré;

- le dossier de conduite;

- l'age, le sexe et le statut civil;

- le groupe de tarification du véhicule.

Autre fait à noter; la réduction enregistrée dans les règlements de sinistres depuis quelques années a été effacée par la hausse des frais d'administration des compagnies d'assurances. (Voir Tableau No 1 ) 
TABLEAU 1

FRAIS D'EXPLOITATION DES ASSUREURS EN POURCENTAGE DES

PRIMES SOUSCRITES AU QUEBEC

\begin{tabular}{llllll} 
Année Commissions & $\begin{array}{c}\text { Taxes } \\
\text { et } \\
\text { permis }\end{array}$ & $\begin{array}{c}\text { Règlement } \\
\text { des }\end{array}$ \\
\hline 1972 & $12,1 \%$ & $2,2 \%$ & $3,6 \%$ & $8,5 \%$ & $26,4 \%$ \\
1973 & 12,2 & 2,4 & 3,5 & 8,2 & 26,3 \\
1974 & 11,5 & 2,1 & 3,7 & 8,6 & 25,9 \\
1975 & 11,3 & 2,3 & 3,3 & 7,1 & 24,0 \\
1976 & 11,3 & 2,3 & 3,1 & 6,8 & 23,5 \\
1977 & 11,7 & 2,3 & 3,7 & 7,4 & 25,1 \\
1978 & 12,4 & 2,4 & 4,2 & 9,3 & 28,3 \\
1979 & 12,4 & 2,6 & 4,2 & 9,5 & 28,7 \\
1986 & 13,1 & 3,3 & 4,0 & 9,4 & 29,8 \\
1981 & 11,6 & 3,3 & 3,7 & 9,7 & 28,3
\end{tabular}

* Excluant les frais des experts en sinistres salariés

Sources: Rapport du Surintendant des Assurances, 1982

P. 37 


\section{$2.2 .3 .3-$ Les estimateurs privés}

Cette catégorie qui devait en principe disparaitre avec l'avènement des centres d'estimation a été maintenue par la force des choses. A titre d'exemple, le Saguenay Lac St-Jean ne compte quiun centre situé a Chicoutimi. Il devenait alors utopique de penser à remorquer tous les véhicules à cet endroit ou encore, que les employés du centre puissent couvrir toute la région de façon efficace.

Les estimateurs privés ont continué leurs travaux et encore là, certains abusent de leurs fonctions pour tenter de respecter les consignes des compagnies d'assurance et donc de préserver leurs emplois. Les problèmes soulevés dans la section précédente se rencontrent aussi ici.

\section{$2.2 .3 .4-$ Les fournisseurs}

Dans le domaine du débosselage, l'achat de matières premières et de services n'est pas un item majeur, le plus important demeurant le coot de la main d'oeuvre. D'après les états financiers consultés et 
suite aux discussions avec les membres du groupe de travail, ceci peut constituer aux environs de $10 \%$ des coots d'opération.

Les principaux produits ou services achetés sont les suivants:

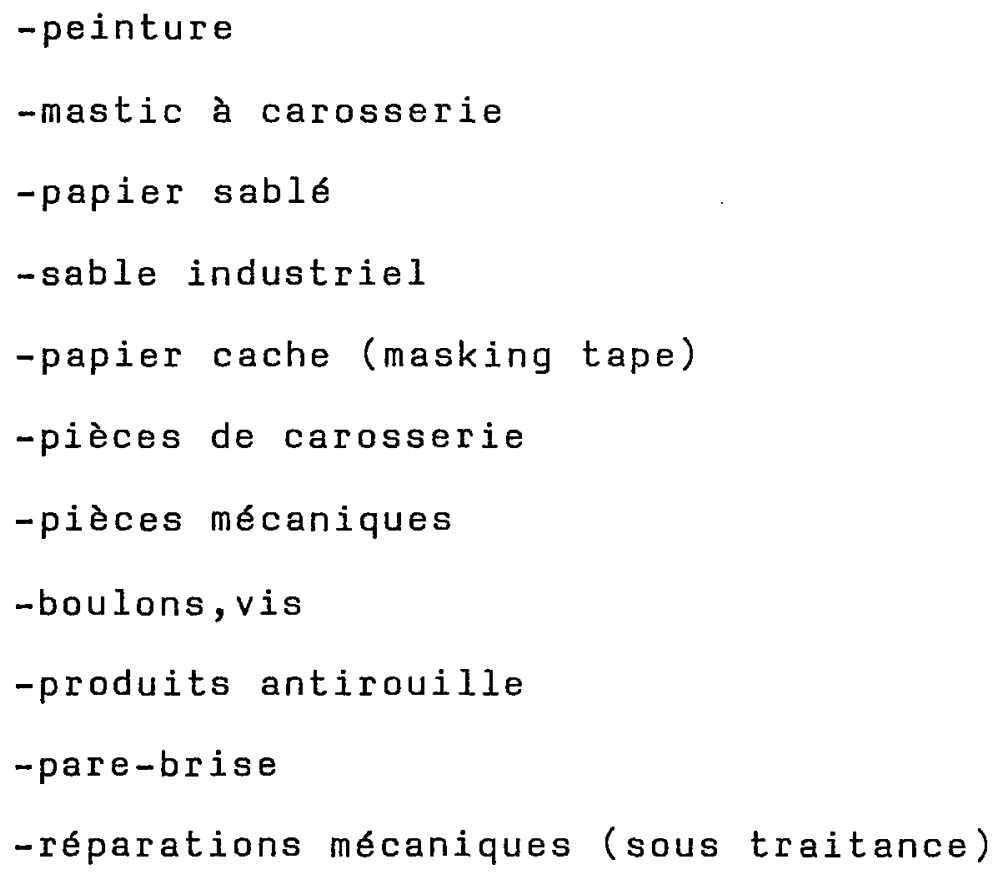


Les ateliers n'ont pas de traitement de faveur et obtiennent les escomptes courants qui sont de $15 \%$ chez les grossistes et entre $10 \%$ et $20 \%$ chez les concessionnaires. Actuellement, certains fournisseurs ont entrepris de diminuer ces escomptes pour améliorer la rentabilité. Or, les démarches effectuées par quelques débosseleurs individuellement ont permis de constater que seule une action collective pourrait madifier la situation.

Au niveau de l'approvisionnement, il ne semble pas exister de difficultés majeures sauf dans le cas de pièces automobiles particulières surtout pour les modèles récents. Enfin, mentionnons que le nombre de fournisseurs est raisonnable et qu'il y aurait surement lieu d'envisager des démarches regroupées pour négocier des ententes particulières.

Nous terminerons cette section en soulignant que les fournisseurs encouragent les garages clandestins puiqu'ils leur vendent tous les matériaux requis avec souvent le même escompte. 


\subsection{4- LE MARCHE}

Dans cette section, nous élaborerons sur l'environnement économique propre à l'industrie du débosselage au Saguenay. L'étude sera présentée selon quatre volets: l'analyse de l'offre et de la demande, la concurrence, les compagnies d'assurance et les fournisseurs de biens et de services reliés à l'industrie.

\subsubsection{1- Analyse de l'offre et de la demande}

Afin de bien cerner le contexte économique de cette industrie, nous allons présenter l'importance de l'activité enregistrée au cours des dernières années. Ceci nous permettra de voir les tendances qui se dégagent en terme de l'offre et de la demande.

\section{$2.2 .4 .2-\underline{\text { La demande }}$}

Basée sur les chiffres mentionnés au tableau 2, il appert que la demande pour ce service s'est accrue au cours des dernières années. Plusieurs constatations se dégagent pour expliquer cette tendance. 
Premierement, la crise économique que nous avons connue particulièrement entre 1980 et 1982 a fait en sorte que la vente d'automobiles neuves a diminué considérablement puisque,les consommateurs autant que les entreprises, n'avaient plus la disponibilité requise pour effectuer ces achats. Egalement, le prix moyen des véhicules a connu des hausses importantes durant cette même période.

Deuxièmement, et ceci est la résultante de la première, les consommateurs ont préféré conserver leurs automobiles en investissant plutot dans leur restauration ou encore en achetant des véhicules usagés nécessitant des. oréparations effectués par le commerçant-vendeur ou l'acquéreur.

Troisièmement, l'apparition généralisée de véhicules de dimensions réduites pour contrer les coûts d'opération a amené la situation où, en cas de collision, ces memes automobiles se trouvaient endommagées pour des montants plus importants. 
TABLEAU NO 2

EVOLUTION DU MARCHE DU DEBOSSELAGE AU SAGUENAY

ANNEE

Exécutant

Ateliers de

Débosselage

Concessionnaires

Automobiles

Ateliers

Clandestins

Total

$16,000,000$

$2,000,000$
1982

$15,250,000$

$13,000,000$

$1,000,000$.

$1,500,000$.

$2,750,000$.

Source: D'après un sondage effectué auprès des ateliers de débosselage et concessionnaires automobiles et des données fournies par le comité paritaire de l'automobile. 
Cette dernière hypothèse fait suite à des vérifications effectuées au Centre d'Estimation du Saguenay lequel a confirmé que le prix moyen d'une réclamation avait augmenté au cours des dernières années pour cette raison.

Par contre, l'entrée en vigeur de la nouvelle loi de l'assurance automobile en 1977 et la revision sur des bases plus sévères du code de sécurité routière en 1980, a eu un effet positif sur le nombre de véhicules impliqués dans des collisions de la route. Quoique cette réduction ait été moins sentie au Saguenay, elle a eu néanmoins un impact sur l'industrie du débosselage.

La nouvelle stratégie des grands de l'automobile d'offrir de meilleures garanties sur leurs véhicules quand aux problèmes de rouille, a modifié la position des ateliers indépendants. En effet, plusieurs travaux maintenant couverts par ces garanties, sont effectués par les concessionnaires automobiles.

Finalement, selon les propriétaires d'ateliers particulièrement ceux possédant des remorqueuses, les hivers plus cléments ont enlevé un bon volume de réparations quant aux accidents moins fréquents durant cette saison. 


\section{$2 \cdot 2 \cdot 4 \cdot 3$ - L'offre}

Au niveau de l'offre, cette dernière n'a pas connu de variation importante pour la période correspondante. En effet, même si nous avons assisté à l'ouverture d'une vingtaine d'ateliers depuis 1980 , le nombre de fermeture a suivi sensiblement la meme courbe. (D'après les statistiques du Centre d'Estimation du Saguenay)

Selon les renseignements que nous avons obtenus du Centre d'Estimation du Saguenay, la plus récente évaluation effectuée en.mai 1983, faisait état de 85 entreprises accréditées a effectuer des travaux de

- débosselage. De ce nombre, nous avons répertorié 62 entreprises indépendantes et 23 autres intégrées aux concessionnaires automobiles.

Toujours selon les renseignements fournis par le Centre, il semble que l'offre ne soit pas trop élevée ni trop faible en terme de garages doment enregistrés et que, normalement, les profits réalisés sont raisonnables. Les difficultés financières rencontrées par certains, s'expliquent par une sous-capitalisation 
chronique ou par une structure de prix inappropriée.

D'ailleurs, ceci est encore plus évident considérant l'entrée en vigueur du décret régissant les salariés du secteur du débosselage, lequel décret a augmenté considérablement la masse salariale sans contre-partie en terme de hausse de tarifs aux usagers.

Cette situation s'est aussi envenimée avec les nouvelles pratiques des compagnies d'assurance. Par leur chantage, elles forcent les ateliers (surtout les plus petits) à baisser leur prix par crainte de perdre le travail. Cette constatation est toujours revenue lors des nombreuses réunions que nous avans eues avec les propriétaires d'atelier ou avec le bureau de direction de l'Association.

Il faudra cependant que les autorités fassent en sorte de ne pas augmenter significativement le nombre de permis du moins pour trois à cinq ans. Cela permettra aux débosseleurs de consolider ou améliorer leurs positions financières et de voir si les tendances du marché mentionnées se maintiendront. 


\section{$2.2 .4 .4-$ La concurrence}

En plus de la concurrence déloyale effectuée par les ateliers clandestins, il existe deux autres types de concurrence soient: la concurrence des concessionnaires automobiles et la concurrence des membres entre eux.

\section{2 .4 .5 - Les concessionnaires automobiles}

Ce type de concurrence a commencé à connaitre son importance depuis environ deux ans. Jusque la, les concessionnaires observaient une tarification d'au moins $20 \%$ supérieure à l'ensemble des débosseleurs (il suffit de faire le tour de ces garages pour constater le tarif horaire affiché lequel est normalement le même que pour les autres réparations automobiles). Ils avaient donc uniquement une petite part du marché. Avec la crise cependant, ils ont pour la plupart, commencé à réduire leurs frais généraux pour, par la suite, diminuer leurs tarifs allant parfois plus bas que l'ensemble des petits débosseleurs. Ils ont aussi, dans certains cas, négocié des contrats fermes avec des compagnies d'assurance pour 
effectuer les travaux sur tous les véhicules accidentés et couverts par ces compagnies.

De plus, l'apparition de garanties plus longues de la part des manufacturiers automobiles a apporté du travail additionnel à ces garages.

Cependant, compte tenu des restrictions inhérentes à ces garanties et le nombre restreint de consommateurs se prévalant de celles-ci, nous ne prévoyons pas une hausse trop importante de ces concurrents puisque, de toute façon, ils devront revenir à des niveaux de prix plus en relation avec leur structures administratives plus dispendieuses que celle des petits ateliers. Aussi, suite au récent conflit de travail dans ces garages, plusieurs ont décidé de tout simplement fermer leurs ateliers de dóbosselage.

\section{$2.2 .3 .2-$ Les clandestins}

Ne répondant à aucune désignation légale, ces ateliers ou individus effectuent, dans des conditions souvent inacceptables, du travail de débosselage en sous-main et viennent perturber l'offre pour ces 
services. Ces ateliers, qui ont connu un essor considérable en raison de la crise économique, regroupent des travailleurs qui sont souvent prestataires de l'assurance chômage ou de l'aide sociale et qui arrondissent leurs budgets par ces travaux non déclarés. Comme les procédures de contrôle et de dénonciation sont très difficiles à appliquer, il est impossible pour l'instant de fermer ces ateliers de façon permanente.

Les activités de ces ateliers sont concentrées dans les travaux de rouille généralement sur des véhicules agés. Ils n'effectuent pas encore, pour le moment, des travaux couverts par des réclamations d'assurance.

Il y a donc lieu d'entreprendre des efforts importants pour tenter de contrer ce problème. Il sera surement souhaitable d'exercer des pressions sur le comité paritaire afin qu'il s'attaque en priorité à cette difficulté comme l'ont exprimé les officiers lors d'une assemblée générale d'information tenue le 31 mai 1983 à Chicoutimi. 


\section{2 .5 LE CONTEXTE LEGAL}

L'industrie du débosselage existe depuis l'apparition des véhicules automobiles soit le début du XXième siècle. Au début, et jusqu'à la fin des années 50, il n'existait pas de législation spécifique qui régissait les activités de ce secteur économique. Ce métier connu initialement sous le nom de carrossier a commencé à se développer par l'entremise des garages engagés dans la vente d'automobiles et de camions. La formation s'effectuait sur le tas et c'est d'ailleurs à partir de l'expérience acquise par les gens du milieu que le système d'éducation actuel a mis sur pied des programmes de formation technique pour répondre à la demande en main d'oeuvre qualifiée.

Le Québec n'a pas fait exception à la règle et a suivi l'influence américaine tout comme le reste du Canada.

Ainsi, nous avons vu apparaitre sur le marché une main d'oeuvre diplômée même s'il n'existait pas en soi de règle obligatoire de classification. Les propriétaires d'ateliers de débosselage continuaient à 
développer leur personnel selon leurs besoins propres et embauchaient selon leurs disponibilités.

L'industrie est soumise bien sûr à d'autres considérations légales lesquelles ont été croissantes comme ailleurs. Toutefois, c'est dans le secteur du Travail et de l'Environnement que les changements ont été les plus nombreux et donc, générateurs de situations problématiques. Nous allons procéder à la revue de ces lois.

\section{$2.2 .5 .1-$ Lois du travail}

Ce n'est que vers 1956 que l'on a vu apparaitre une législation touchant les débosseleurs. Cette loi portait le nom de: "LOI SUR LES DECRETS DE CONVENTION COLLECTIVE" et avait d'abord été instaurée à la demande de personnes oeuvrant dans le secteur de la coiffure.

Cette loi a, en fait, la même portée qu'une convention collective conclue entre un employeur et les travailleurs d'une entreprise, mais s'étend à un niveau plus complexe. L'application du décret est confiée à un comité paritaire qui, comme son nom l'indique, regroupe 
les employeurs et les associations de travailleurs concernés selon la règle de la parité.

$$
\text { C'est l'instance privilégiée laquelle }
$$

administre les fonds perçus, embauche des inspecteurs pour effectuer les vérifications, met à l'amende les entreprises qui ne respectent pas le décret et, finalement, suggère au Ministre du Travail des modifications à apporter à la loi.

Or, en vertu de l'article 2 de cette même loi, le législateur avait prévu la possibilité de pouvoir décréter qu'une convention collective relative à un métier, à une industrie, à un commerce ou à une profession, lie également tous les salariés et tous les employeurs du Québec ou d'une région déterminée du Québec dans le champ d'application défini dans ce décret.

La procédure pour en faire partie est relativement simple. Il s'agis qu'une des parties contractantes (employeurs ou travailleurs) dépose une demande auprès du Ministre du Travail lequel, s'il juge la position fondée, entreprendra alors les consultations auprès des personnes ou organismes intéressés à se faire 
entendre sur la pertinence du décret demandé. S'il n'y a pas d'éléments nouveaux qui sont apportés au cours de ces auditions publiques, le ministre doit assumer qu'il n'y a pas d'objections à sa mise en application. Il ne reste alors qu'a déposer la requête au Conseil des Ministres et à l'Assemblée Nationale par la suite. Une fois publié dans la gazette officielle, le décret devient exécutoire à la date qui aura été retenue au préalable.

Au Saguenay Lac St-Jean, la première apparition de cette loi eut lieu en 1958 et touchait la région de Dolbeau i.e., le haut du Lac St-Jean jusqu'à Chibougamau. Elle n'avait cependant aucune juridiction sur le reste de la région.

Cependant, durant l'année 1982 , la Corporation des Concessionnaires Automobiles du Saguenay Lac St-Jean était en renégociation de contrat de travail avec ses employés. Sentant survenir un conflit de travail imminent, étant donné l'achoppement des discussions sur plusieurs points dont celui de la classification des débosseleurs oeuvrant dans leurs entreprises respectives, elle demandait au Ministre du Travail 
l'adoption d'un décret en vertu de la Loi des Décrets de Convention Collective. Elle voulait se donner en fait un règlement de classification pour ses employés et mettre ainsi fin à de nombreuses tergiversations.

Les audiences publiques eurent lieu en novembre 1982 à Chicoutimi et, comme il n'y eat aucune opposition de formulée, le Ministre décidait d'acquiesser à la demande. Comme cette loi était en révision et que le gouvernement avait décrété un moratoire sur la création de nouveau comité paritaire, il contournait cette difficulté en extensionnant le territoire du Saguenay au comité paritaire de I'autamobile de Dolbeau. La date de p.rise d'effet fut annoncée pour le 1 juin 1983.

Les principales modifications dans l'industrie du débosselage et les ateliers de débosselage en particulier concernaient les points suivants:

- la semaine de travail;

- la rémunération;

- les congés et vacances;

- la classification. 
Ainsi, tout le secteur du débosselage fut lié par ce nouveau décret. Et il était trop tard pour s'y objecter. Il ne restait plus qu'à obtenir la permission de siéger sur le comité paritaire et de tenter d'apporter des modifications.

\section{$2.2 .5 .2-$ Loi sur l'environnement}

Un autre obligation touchant directement les débosseleurs fit son entrée progressivement durant les années 1970 au niveau de la qualité de l'environnement. La situation fut confirmée en 1979 avec la LOI SUR LA QUALITE DE L'ENVIRONNEMENT.

Les modifications avaient trait aux spécifications relatives à la construction des chambres de peinture, à l'utilisation du sablage industriel dans la préparation des véhicules pour la peinture et le niveau de bruit limite acceptale.

Cela impliquait une augmentation dans les couts de construction puisque les chambres de peinture ne pouvaient plus seulement être constitués d'un espace fermé à l'intérieur de l'atelier mais bien d'une unité ayant des spécifications précises pour l'évacuation de 
l'air et pour l'étanchéité de la pièce. Il en est de même pour l'utilisation du sablage industriel à ciel ouvert et le niveau de bruit des différentes opérations.

A titre d'exemple, avec l'entrée en vigeur de cette loi, une chambre de peinture qui cootait normalement entre $\$ 10,000$. et $\$ 15,000$. vit la facture monter jusqu'à $\$ 30,000$. et $\$ 40,000 \ldots$

\section{$2 \cdot 2 \cdot 5 \cdot 3$ - Autres Iois}

Dutre les lois que nous venons de mentionner, les débosseleurs sont soumis à une foule d'autres lois comme pour les autres types d'entreprises. Sans en faire une liste complète, nous pouvons énumérer les suivantes:

a: Lois des Sociétés Commerciales Québéçoise ou Canadienne;

b: Lois de l'impôt provincial et fédéral;

c: Loi relative aux licences d'affaire;

d: Loi relative à la taxe de vente;

e: Loi d'urbanisme;

f: Loi de Santé et Sécurité. 


\title{
2.2.6 LE FONCTIONNEMENT DE L'INDUSTRIE
}

\begin{abstract}
Nous aborderons maintenant l'étude des activités réalisées à l'intérieur de l'industrie. La méthode utilisée sera celle des flux afin d'établir les relations existant entre les activités, d'identifier si possible les incohérences entre celles-ci et enfin, de voir les activités qui pourraient être manquantes.
\end{abstract}

\subsubsection{1- Système global de l'industrie}

(Voir Schéma No 2 )

Prenant en considération le client privilégié dans ce mémoire, le système global de gestion et de transformation que nous avons identifié dans le domaine du débosselage regroupe les activités de trois secteurs soient:

- l'octroi des contrats de débosselage;

- I'exécution de ces mêmes contrats;

-l'administration des ateliers. 
SCHEHA NO 2

\section{PROCESSUS DE GESTION ET TRANSFORHATION INDUSTRIE DU DEBOSSELAGE}

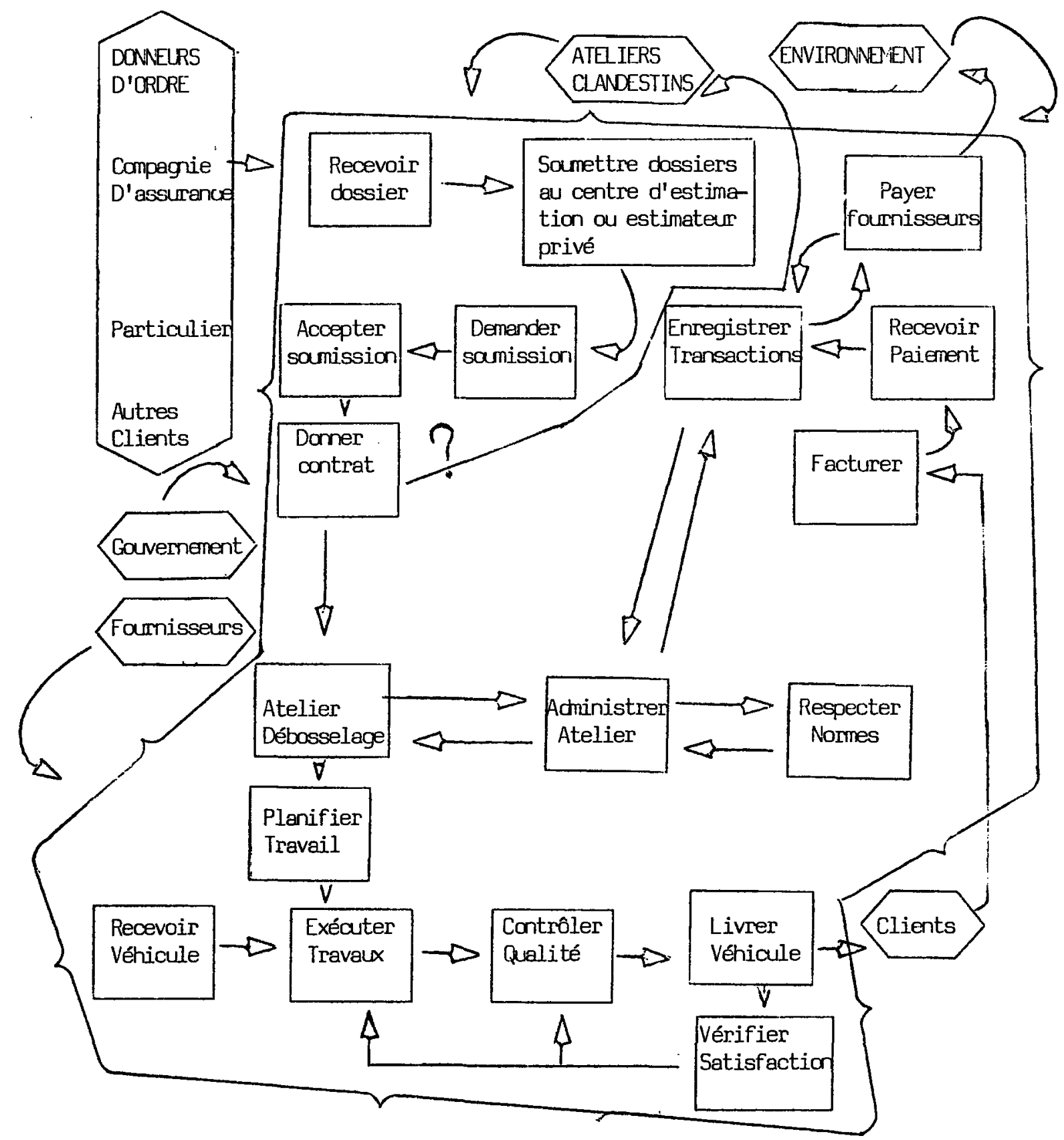


Le premier sous-système regroupe les activités efectuées par tous les intervenants et démontre la relation d'autorité qui prime de la part des donneurs d'ordres particulierement les compagnies d'assurance. Tout ce processus a d'ailleurs été mis sur pied pour répondre aux impératifs de cette catégorie, a servi de base de référence aux autres utilisateurs identifiés et a été imposé, bon gré mal gré, au reste de la structure précédemment énoncée.

C'est la forme généralisée qui prévaut à l'échelle du Québec et par conséquent au Saguenay. Comme nous le mentionnions dans une section précédente,la raison majeure de cette façon de procéder est née du désir qu'ont éprouvé les assureurs de tenter de restreindre les coats d'opérations qui les touchent directement respectant par là, le voeu du Surintendant des Assurances qui y voyait un moyen de limiter les hausses de primes d'assurances.

Toutefois, il ne semble pas que cela ait connu le succès escompté puisque, dans son dernier rapport annuel, le Surintendant continue à demander aux compagnies de s'efforcer de réduire les hausses 
annuelles. Evidemment, il s'agit d'un voeu politique souhaitable pour la collectivité mais qui ne semble pas encore se réaliser.

Les deux autres sous-systèmes quant à eux, touchent plus particulièrement les activités de gestion et de transformation de la section inférieure de l'organigramme i.e. les ateliers de débosselage. C'est la structure utilisée par l'ensemble de ces intervenants quoiqu'a des degrés de raffinements différents dépendamment de la taille et de la spécialisation de chacun des ateliers.

\section{$2.2 .7-$ LES SQUS-SYSTEMES}

Prenant en considération l'évolution particulière de l'industrie depuis quelques années, notre systeme-client nous a demandé de nous attarder particulièrement à l'octroi des contrats. Cette orientation provient du fait que les difficultés rencontrées actuellement dans l'industrie concernent surtout cet aspect et qu'il constitue la pierre angulaire de tout ce secteur d'activité économique. 


\section{SCHEMA NO 3}

\section{SOUS-SYSTEME D'OCTROI DE CONTRAT DE DEBOSSELAGE}

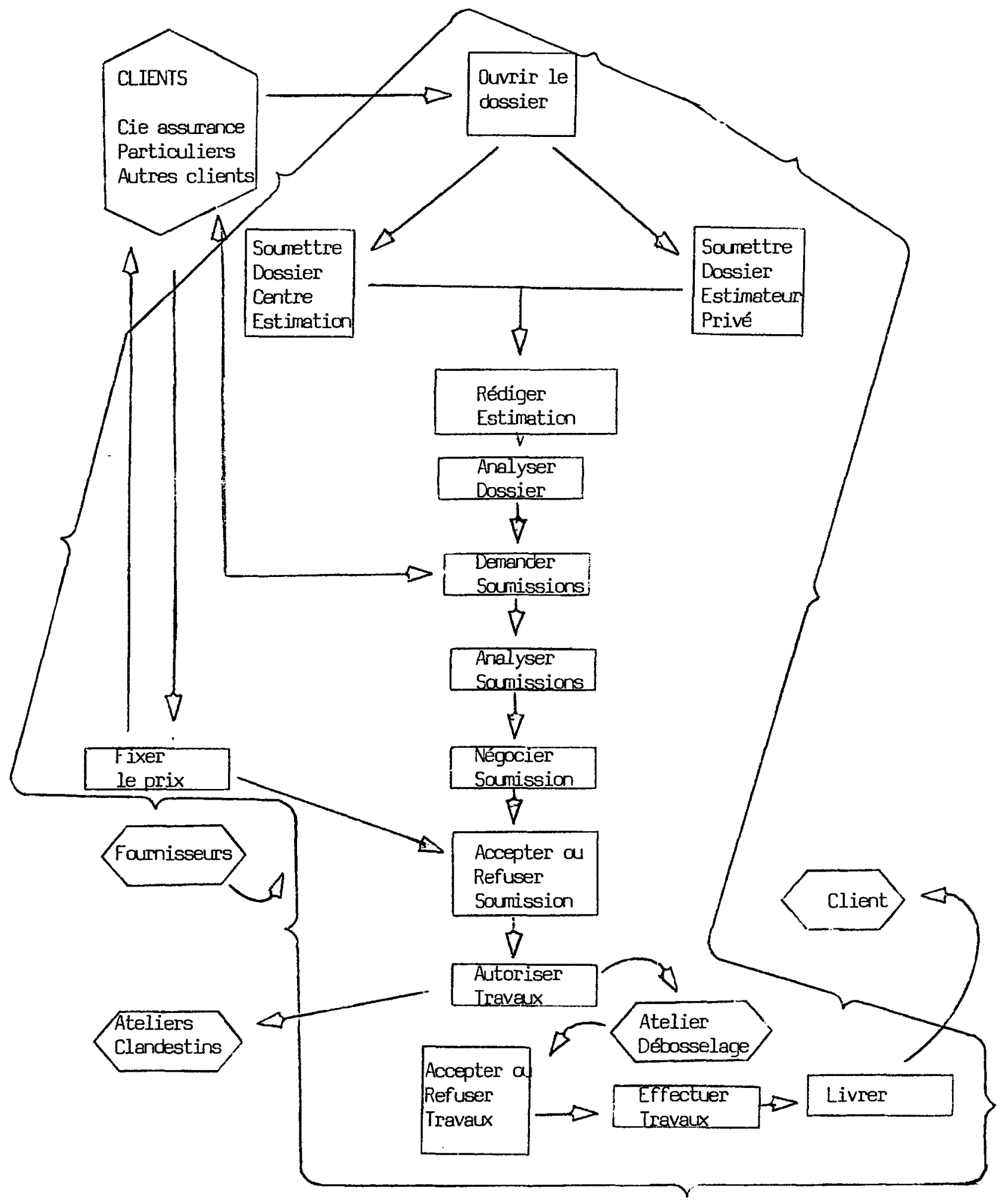




\subsubsection{1- Sous-système octroi de contrats \\ (Voir schéma No 3 )}

Le rôle de ce sous-système est de permettre d'accorder les travaux de débosselage aux ateliers selon les normes établies dans un délai le plus court possible. Il implique normalement la collaboration de toutes les parties pour arriver a satisfaire le client rapidement et au meilleur prix.

Pour rencontrer son mandat, le sous-système doit tenir compte des objectifs stratégiques et opérationnels des intervenants, des forces et des faiblesses des parties et de l'environnement avec lequel ses activités sont en relation.

Le fonctionnement normal de ce sous-système est handicapé par trois problématiques. La première concerne la difficulté de marier les objectifs spécifiques de toutes les parties dont surtout ceux des assureurs et ceux des débosseleurs. Dans le cas des assureurs, nous parlons des objectifs de rentabilité dans le sens de meilleur prix. Pour les ateliers de débosselage, nous parlons de rentabilité au sens de protéger la tarification existante pour faire face aux hausses de prix pour la main d'oeuvre et les matières premières. 
La seconde problématique réside dans le rapport de force qui prévaut entre ces deux groupes. D'une part, les compagnies d'assurance qui font front commun et qui ont standardisé les opérations dans ce domaine et, d'autre part, les ateliers de débosselage qui réagisent de façon individuelle et qui tentent par tous les moyens de sauver ce qu'ils ont en terme de droits acquis.

Finalement, l'entrée en vigeur d'une nouvelle législation par le ministère du travail, régissant les activités des ateliers de débosselage au moyen de décret depuis le premier juin 1983 avec l'imposition d'un règlement de classification, une politique de rémunération plus exigeante sans pour autant statuer sur le tarif horaire minimum qui devrait être appliqué.

\subsubsection{2- Processus de réalisation des travaux}

Les étapes de réalisation des travaux de débosselage sont représentées dans le schéma No 4 . Nous $y$ retrouvons le processus traditionnel requis pour la réparation d'un véhicule quelconque. La démarche est la même pour tous les genres de travaux et I'ajustement est fonction de I'importance du travail à être effectué. 


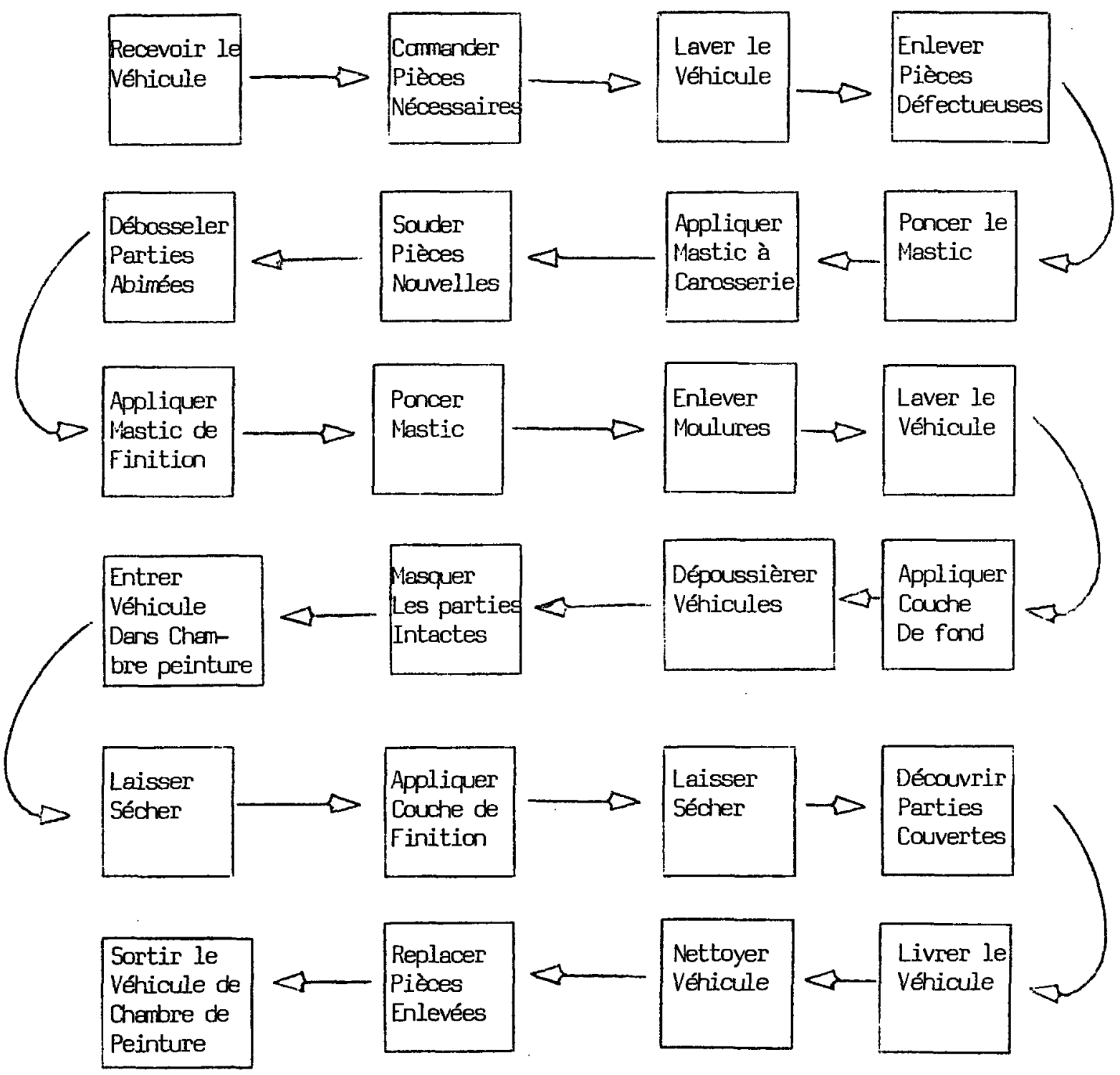


Celui-ci est assez standardisé parmi tous les ateliers de débosselage. Seul le nombre d'activités accomplies par les travailleurs differe selon le nombre d'employés qui y travaillent. Ainsi, par exemple, dans un atelier de 12 à 15 personnes, on retrouvera une spécialisation accrue des taches par opposition aux petits ateliers de 2 ou 3 personnes.

Selon les informations qu'il nous a été donné de recueillir sur le sujet, il ne nous est pas apparu de difficultés particulières dans ce processus. La main d'oeuvre est disponible, généralement bien formée et les équipements adéquats.

\subsubsection{3- Le sous-systèmes de gestion}

Le schéma No 5 présente les principales activités de gestion rencontrées dans le sous-système de gestion d'un atelier de débosselage. Compte tenu de la taille de ces entreprises, les activités décrites sont habituellement réalisées par la même personne, le propriétaire ou, dans certains gros ateliers, par le gérant ou le controleur. 
SCHEMA NO 5

SOUS-SYSTEME GESTION D'ATELIER

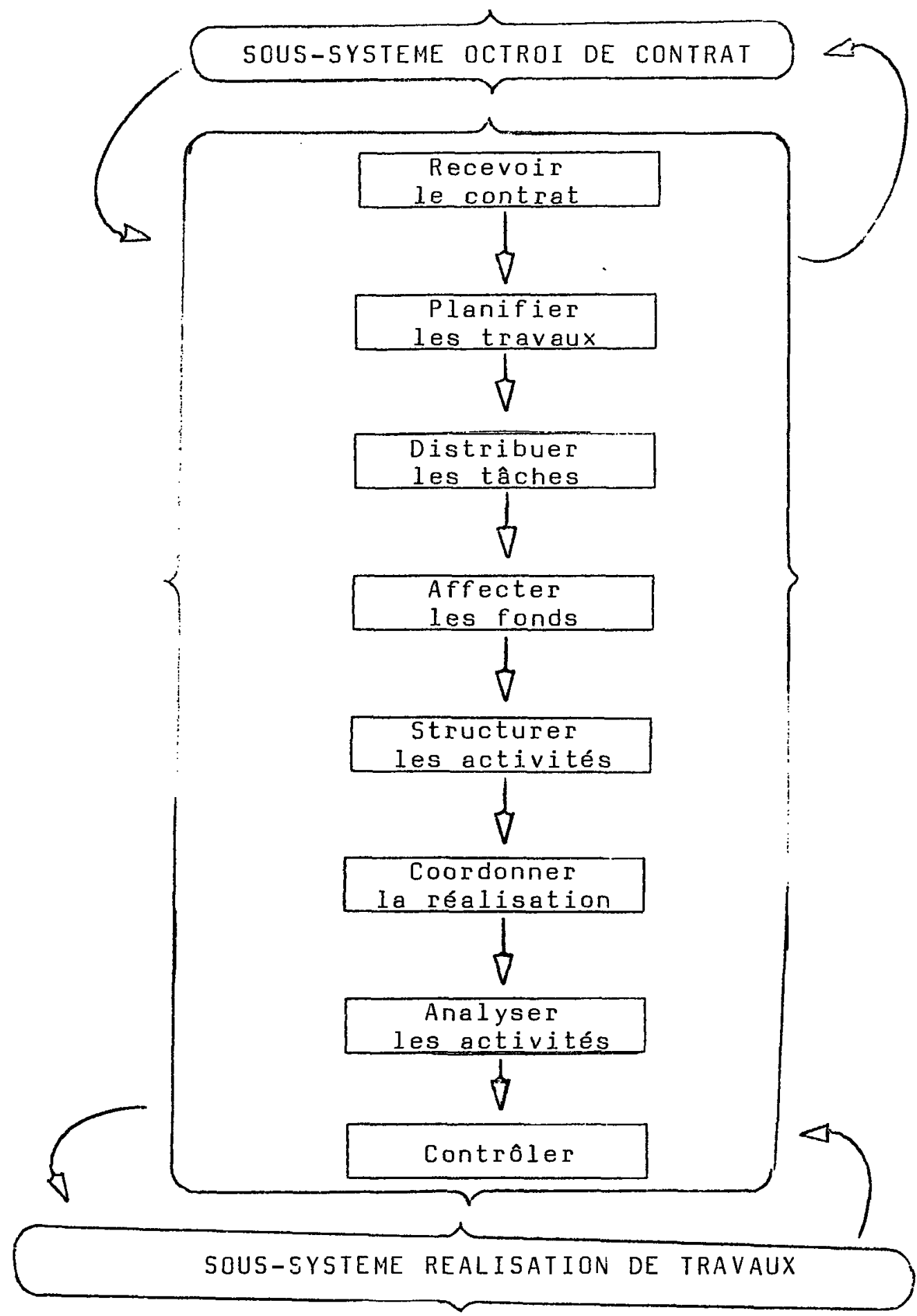


Ici encore, nous n'avons pas identifié de problèmes majeurs sauf pour les très petits ateliers qui négligent les activités d'affectation des fonds et d'analyse des résultats. Or, comme nous l'avons dit, plusieurs coupent leurs prix de façon importante. Ils ne réalisent que trop tard les difficultés que cela occasionne pouvant aller jusqu'à la fermeture de l'atelier.

\subsubsection{4- Commentaires}

A la lumière du processus, nous constatons que l'ensemble des activités identifiées sont clairement définies, qu'elles sont intégrées en sous-systèmes bien structurés cadrant dans un système fonctionnel. L'ensemble de la problématique qui en découle provient des relations qui existent entre les intervenants eux-mêmes et l'environnment en général et à ce chapitre nous notons les gouvernements et les ateliers clandestins. 


\subsubsection{LES PERFORMANCES DE L'INDUSTRIE}

Les communications entre les différentes structures de I'industrie du débosselage sont existantes mais elles sont perturbées par la différence de pouvoir entre les donneurs d'ordres et les exécutants de travaux en particulier, les ateliers de débosselage indépendants.

Les compagnies d'assurance sont regroupées en association sous la responsabilité du Bureau d'Assurance du Canada et observent sensiblement les mêmes politiques pour l'octroi de travaux à être effectués.

A l'opposé, les ateliers de débosselage, malgré certaines tentatives au cours des années, n'ont pas encore réussi à se regrouper pour faire entendre leurs demandes auprès des nombreux intervenants du domaine et les différentes instances gouvernementales.

Avec l'apparition du nouveau décret régissant les activités du débosselage depuis juin 1983, les consultations effectuées auprès de 25 ateliers de débosselage ont permis de constater le manque d'information de plusieurs et la nécessité d'apporter des changements pour les autres. 
Toutefois, pour pouvoir espérer apporter des modifications au décret, il est nécessaire d'être représenté sur le comité paritaire chargé de son application.

Seules les associations patronnales ou syndicales peuvent déposer une demande auprès du Ministre du Travail afin d'être nommés parties contractantes au décret. Les ateliers individuellement n'ont pas la possibilité de le faire.

Les compagnies d'assurance règnent en maitres sur l'industrie faute d'avoir une certaine forme d'opposition de la part des autres parties constituantes.

Les ateliers clandestins constituent un problème majeur pour tous les ateliers de débosselage et, pour améliorer la situation, une action commune doit s'amorcer avec le concours du comité paritaire.

La hausse de frais d'exploitation associée à une réduction du tarif horaire depuis deux ans expose les ateliers à des problemes de survie et suppose des mesures correctives à entreprendre rapidement par l'ensemble des ateliers. 


\section{$2.2 .6-$ LE CLIMAT}

Le climat général de l'industrie est pour l'instant perturbé par ces difficultés de communication que nous avons mentionnées précédemment, ce qui affecte principalement les ateliers de débosselage. Ces derniers se sentent exploités à la fois par leurs meilleurs clients, les assureurs, et par le gouvernement avec ses lois nouvellement mises en vigeur. Les propriétaires d'ateliers sont démunis et voient mal comment ils pourront, sur une base individuelle, améliorer la situation.

Il en est de même pour les centres d'estimation qui, tout en respectant les consignes mises de l'avant par le groupement des assureurs, trouvent difficile de faire les négociations et de subir les écarts entre ceux-ci et les débosseleurs. 


\section{3- SELECTION DU PROBLEME A ETUDIER}

\subsection{1- Liste des candidats aux problèmes}

L'ensemble des symptômes énoncés dans la section précédente laisse sous-entendre les problèmes suivants :

1.- L'absence d'une structure d'association propre aux ateliers de débosselage empêchent l'établissement d'un rapport de force essentiel dans les négociations entre ceux-ci et les autres intervenants du secteur;

2.- Le sous-système d'octroi des contrats actuellement en vigeur avantage les compagnies d'assurance aux détriments des ateliers de débosselage;

3.- Le nouveau décret pénalise les ateliers de débosselage et ils ne peuvent siéger sur le comité paritaire individuellement. 


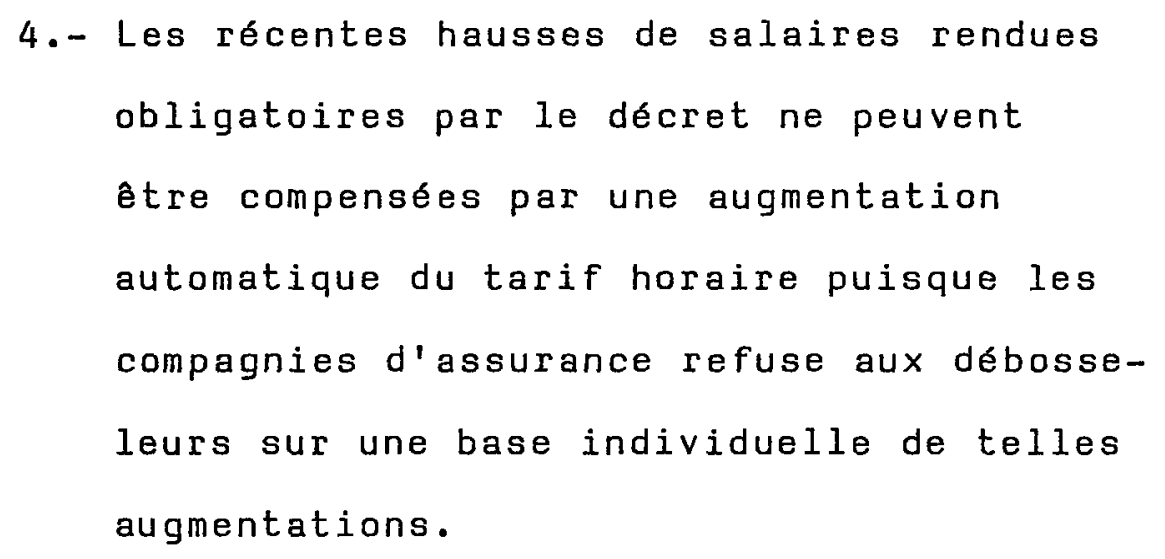

\subsection{2- Sélection et justification du problème}

Pour le système client que nous avions choisi de privilégier dans ce mémoire et suite aux travaux effectués avec le groupe de propriétaires, il appert que le problème majeur est le premier identifié. D'une part, il faut que les ateliers puissent négocier les tarifs et les procédures de règlement avec les compagnies d'assurance et les centres d'estimation. Or, ces deux intervenants refusent, pour des raisons évidentes, de le faire sur une base individuelle.

D'autre part, la venue du décret le premier juin 1983 a apporté des modifications majeures aux conditions d'emploi des travailleurs du débosselage et une augmentation des coats pour les propriétaires 
d'ateliers. De plus, ces propriétaires ne reçoivent pas actuellement d'information sur les obligations s'y rattachant de la part de l'instance chargée de l'application du décret. Seule une association dament accréditée peut faire partie contractante du décret et espérer y apporter des modifications.

D'après les démarches que nous avons effectuées auprès du ministère du travail, des modifications doivent être apportées à la loi sur les décrets de convention collective lors de la prochaine session de l'Assemblée Nationale et une association aura plus de chance d'apporter des suggestions.

Enfin, la conscientisation plus intense de la part des propriétaires d'ateliers de débosselage est actuellement propice à la venue d'une structure de regroupement qui leur permettra enfin de pouvoir se faire entendre. 
CHAP ITRE III

ANCRAGE, MODELE CONCEPTUEL ET COMPARAISON 
CHAP I TRE III

\section{ANCRAGE, MODELE CONCEPTUEL ET COMPARAISON}

\section{1- ANCRAGE}

\subsection{1- Choix du système pertinent}

Le problème retenu est relié à l'absence d'une organisation regroupant tous les ateliers de débosselage du Saguenay.

Nous procéderons donc à l'identification des activités nécessaires à sa mise sur pied et à son fonctionnement.

\subsection{2- Définition du système pertinent}

Le système de regroupement des ateliers de débosselage du Saguenay est un ensemble cohérent d'activités permettant à tous les ateliers de débosselage de se former en association selon le mode qu'ils voudront utiliser afin de permettre à ses 
derniers didentifier les objectifs et les besoins communs, de suggérer des actions précises, de se faire représenter partout où il sera jugé important, afin de pouvoir principalement se donner un véritable pouvoir de négociation auprès des compagnies d'assurances, des centres d'estimation, du comité paritaire de l'automobile et des gouvernements. Il favorisera une saine compétition entre les membres par le respect d'un code d'éthique et des statuts et règlements et en améliorant la qualité des services offerts à l'ensemble de la population. Finalement, il prévoiera des mécanismes susceptibles d'assurer une continuité et un développpement nécessaire à l'exercice d'un rôle rélel dans 1 'industrie.

\subsection{3- Validation}

- Propriétaires

:

Les propriétaires d'ate-

liers de débosselage du Saguenay;

- Environnement

:

Le marché de l'industrie

du débosselage au

Saguenay; 
- Client

- Transformation

- Acteurs

- Point de vue
:

:

Les administrateurs et gestionnaires de l'association;

En se donnant une association, les propriétaires d'ateliers surtout les petits pourront enfin se faire entendre et pourront améliorer leur rentabilité sur une base à long terme. 


\section{2- MODELE CONCEPTUEL}

Le modele conceptuel est constitué d'un ensemble d'activités humaines essentielles déterminées selon un processus déductif à partir des connaissances de l'intervenant et des connaissances disponibles sur le sujet.

Par la suite, les activités qui auront été identifiées seront reliées par des flux pertinents de façon à former le modèle proprement dit, lequel, sera par la suite validé, pour vérifier sa cohérence systémique.

Ce modele conceptuel devra être établi en conformité avec la définition du point d'ancrage retenu.

\subsection{1- Les activités du système conceptuel}

Le système est constitué de cinq activités de base. Ce sont:

- Orientation;

- Structuration;

- Mode de fonctionnement;

- Recrutement; 


\section{MODELE CONCEPTUEL DE REGROUPEMENT}

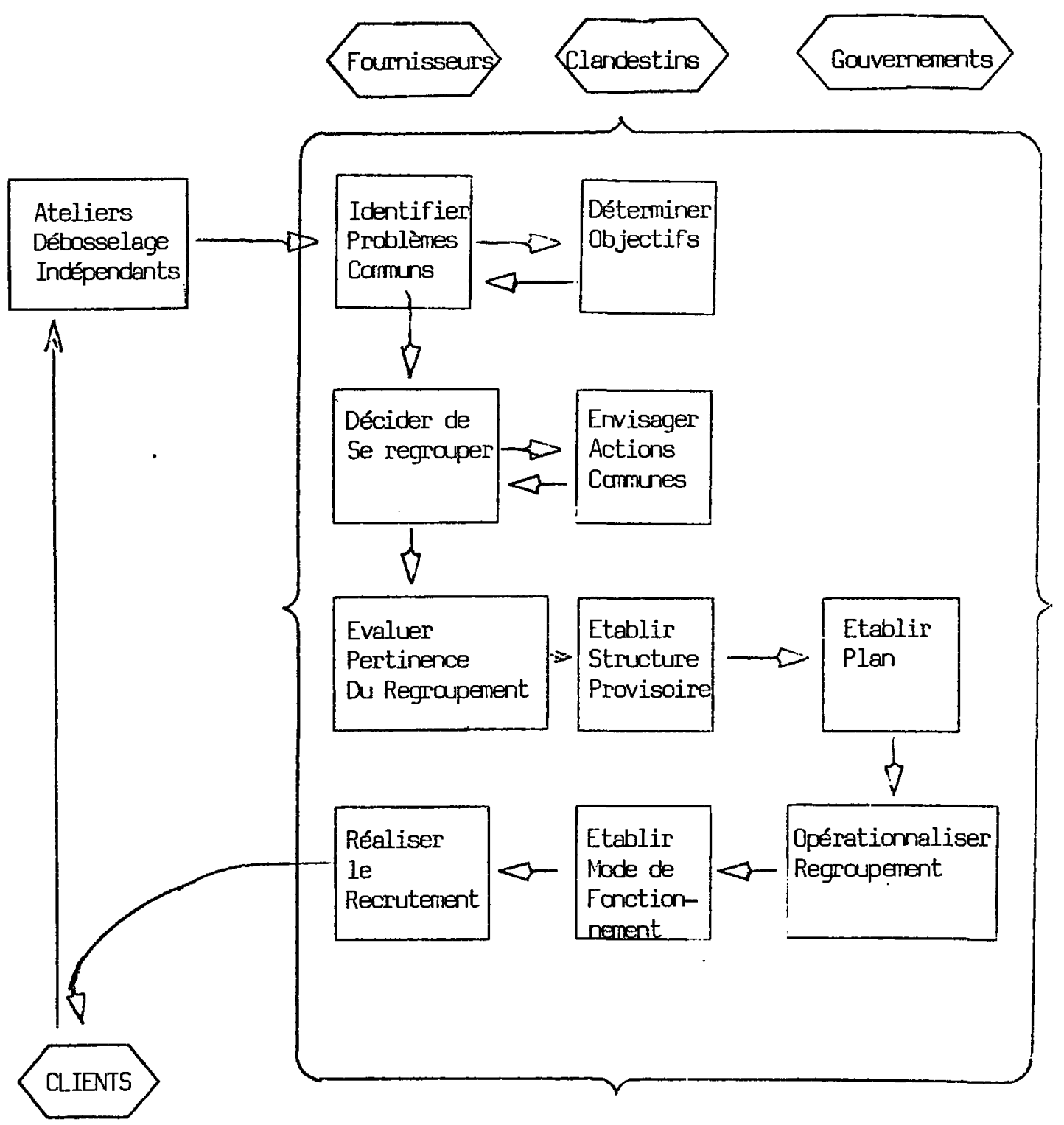


- opérationnalisation.

Le système global est fourni au schéma No 6. Nous allons maintenant étudier en détail chacun des sous-systèmes composant le modèle.

\section{$3.2 .1 .1-$ 0rientation}

Ce sous-système d'activités comprend les démarches préliminaires qui daivent se faire dans la mise sur pied d'un regroupement. Il permet de décider de la pertinence du regroupement, de choisir le genre de regroupement, d'en déterminer les objectifs. II considère également la formation d'une structure d'acceuil provisoire qui verra à entreprendre les actions préliminaires.

Nous reproduisons au schéma NO 7 le sous-système orientation 
SCHEMA NO 7

SOUS-SYSTEME ORIENTATION

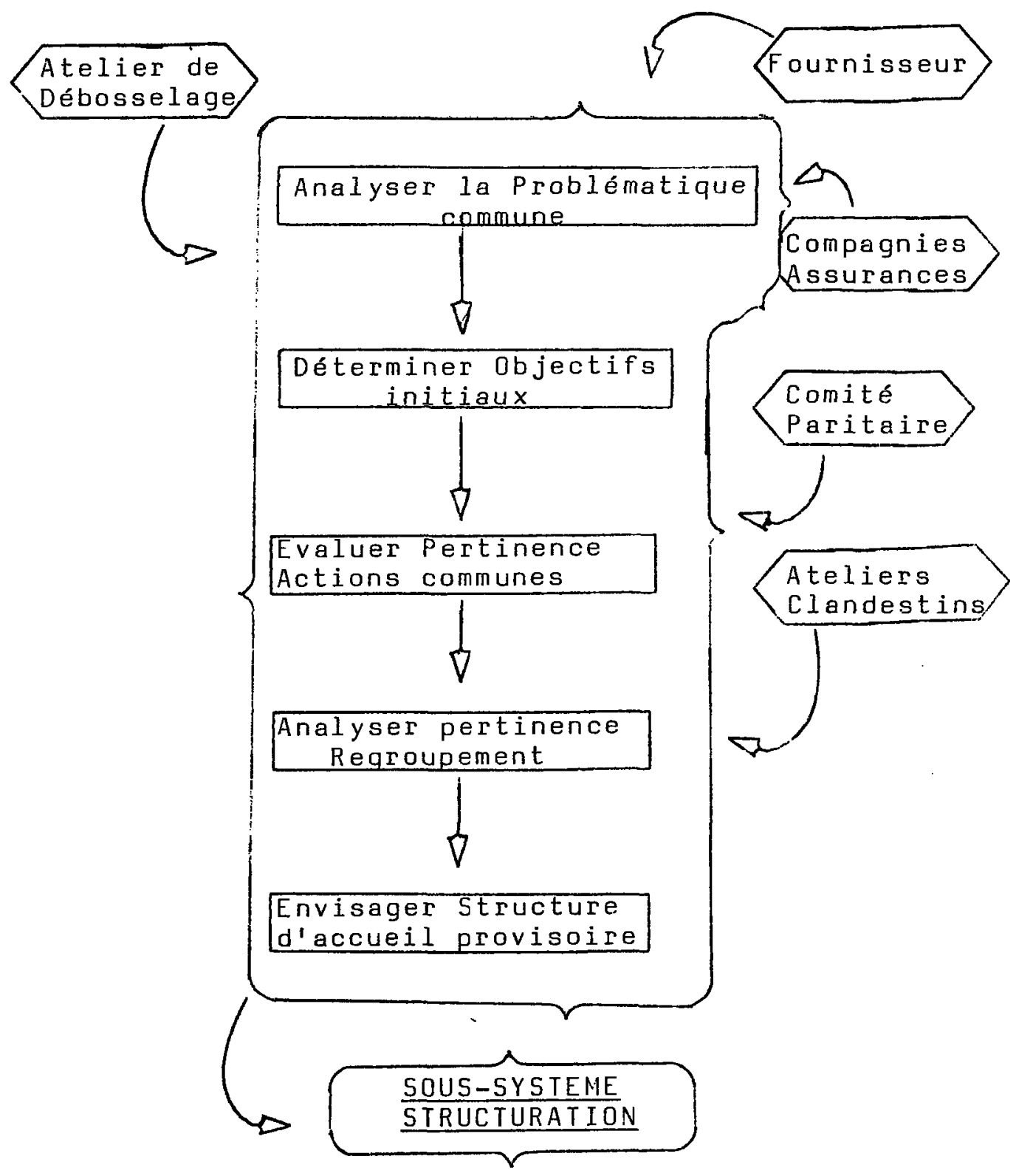




\subsubsection{2- Structuration}

A partir de la formation du comité provisoire, nous entrons dans la deuxième phase qui consiste à donner à l'idée une structure permanente. Dans un premier temps, le comité revise les orientations et les études sur le sujet. Il évalue l'impact politique et économique du regroupement et amorce la définition des services potentiels pour les membres du regroupement. Le processus est présenté au schéma NO 8 .

\subsubsection{3- Mode de fonctionnement}

Dans ce sous-système, nous touchons toutes les étapes nécessaires pour que l'association fonctionne de façon permanente. Les activités vont de la demande de charte s'il y a lieu, à un système d'identification, en passant par l'appel d'une assemblée générale et l'élection du conseil d'administration.

Cette étape peut se réaliser avant ou après le recrutement dépendamment du types d'entreprises ou d'individus que nous voulons regrouper. Sa représentation apparaît au schéma NO 9 . 


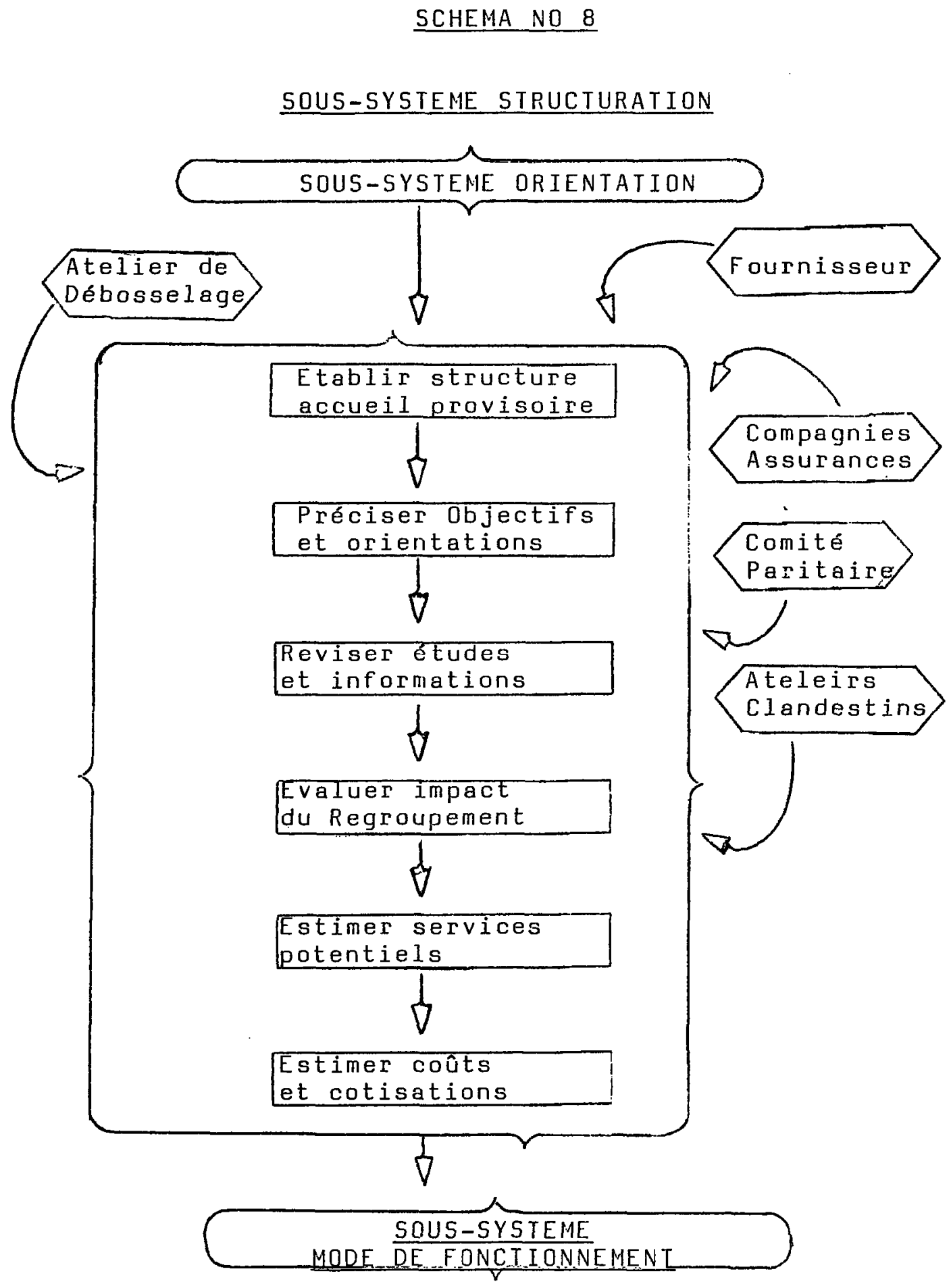




\section{SCHEMA NO 9}

SOUS-SYSTEME MODE DE FONCTIONNEMENT

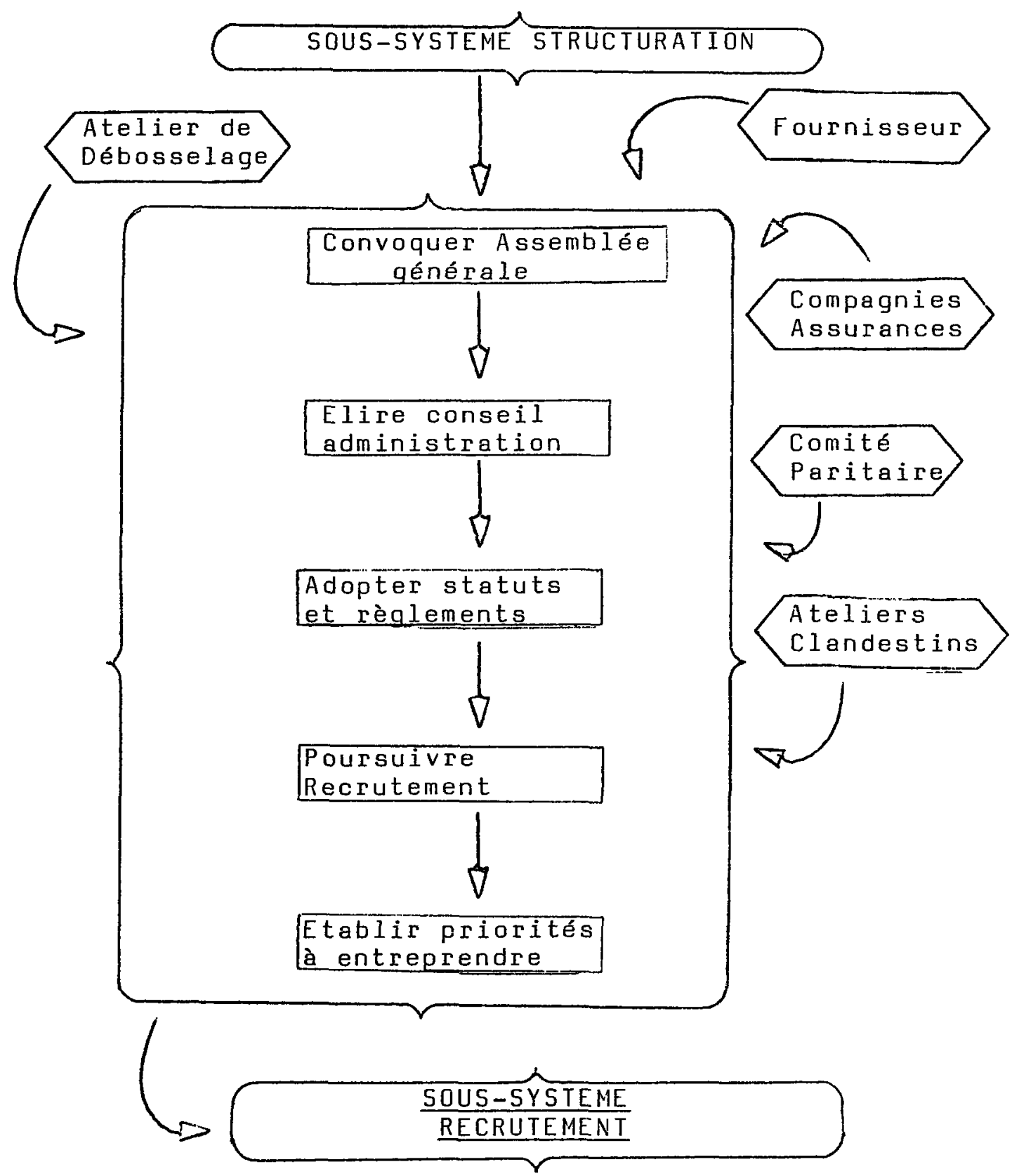




\subsubsection{4- Recrutement}

C'est une étape très importante pour assurer la survie de tout regroupement. Par conséquent, les activités doivent être très bien structurées et le suivi doit être continuel. Ce sous-système comprend l'établissement d'un plan de recrutement, les activités de recrutement et la campagne de recrutement proprement dite.

Un des moyens privilégiés est de susciter l'intérêt des membres potentiels par des économies importantes dans les achats de produits ou services. Evidemment, en procédant de la sorte, nous éliminons les réticences à se regrouper. Toutefois une telle pratique n'est pas utilisable dans notre cas puisque les objectifs premiers du regroupement ont trait à des problèmes techniques et politiques.

Nous avons représenté les activités du recrutement au schéma No 10 . 


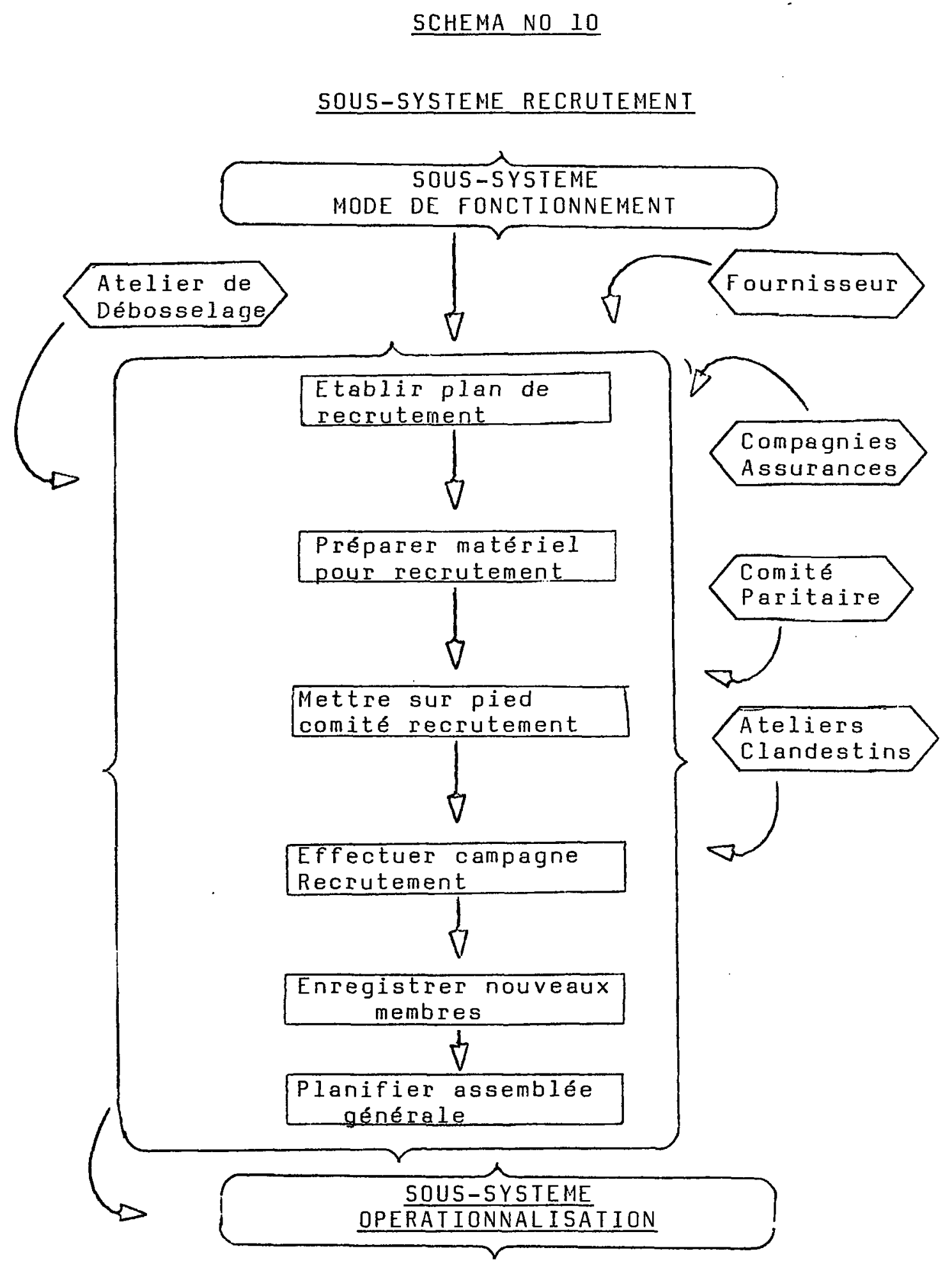




\subsubsection{5- Opérationnalisation}

Cette dernière étape concerne les activités qui doivent être réalisées par le conseil d'administration ou les membres pour donner un sens au regroupement. Elle inclue également la publicité autour de l'association et la promotion de nouveaux services proposés ou demandés par les membres surtout en terme d'activités pouvant garantir davantage la survie de l'association . Le schéma NO 11 vous présente ce dernier sous-système. 


\section{SCHEMA NO 11}

SOUS-SYSTEME OPERATIONNALISATION

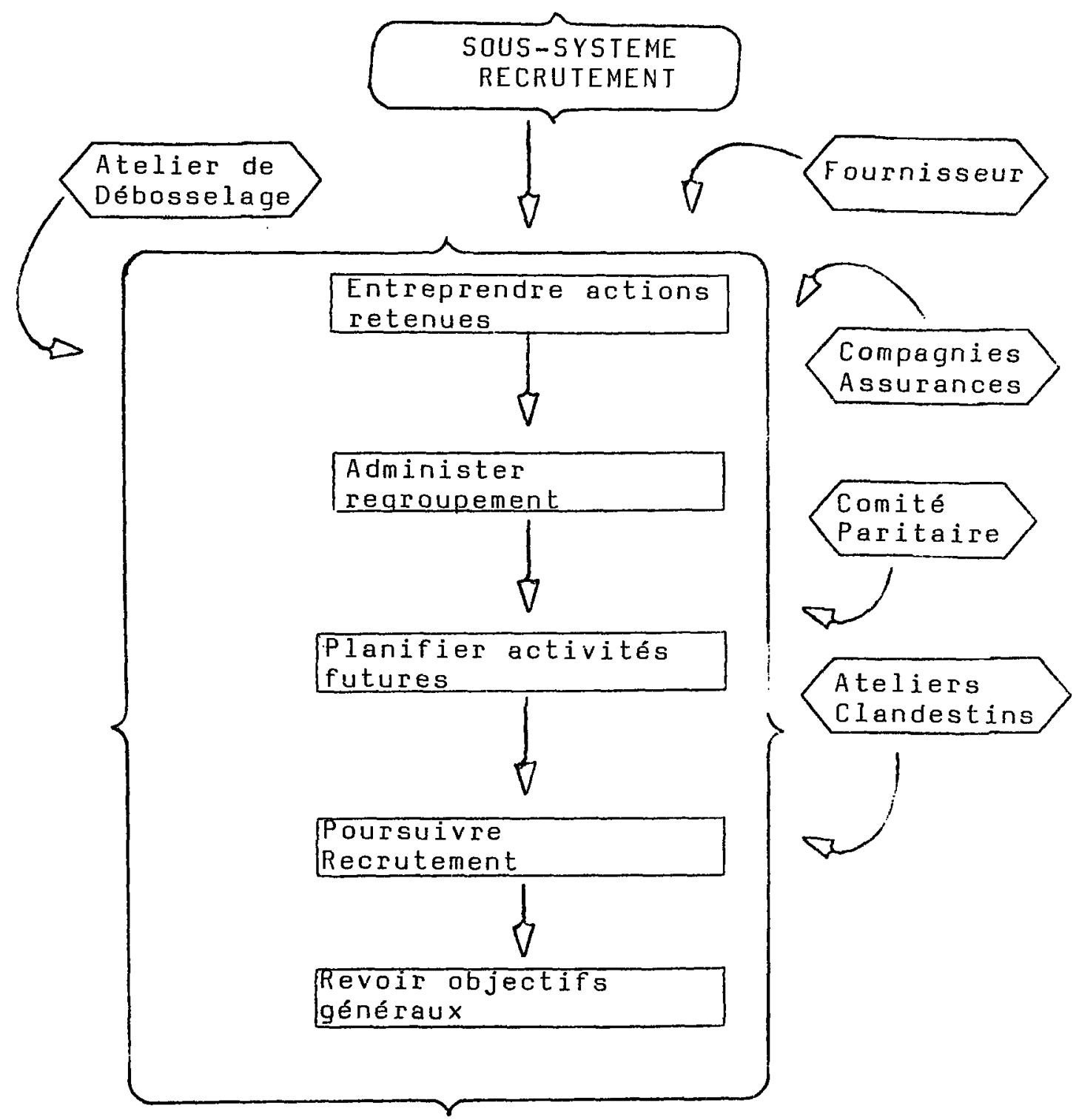




\subsection{2- Validation du modèle conceptuel}

La validation du modele sera réalisée selon les caractéristiques développées par P.B. Checkland soient:

1.- Le système a un objectif, une mission, une fonction;

2.- Le système a des mesures de performance et des mécanismes de contróle;

3.- Le systeme a des composantes elles mêmes, systèmes avec toutes les caractéristiques appropriées;

4.- Les composantes du système ont un certain degré d'interrelation permettant à un effet de se permuter dans tout le système;

5.- Le système est un système ouvert. Il existe dans un système plus large et/ou à un environnement avec lequel il échange. Les frontières d'un système sont définies par les limites du champ d'action des preneurs de décision impliqués;

6.- Le système a des ressources physiques et humaines;

7.- Le système a des preneurs de décision et un processus de décision;

1. Checkland, P.B., Towards a systems - Based methodology for Real World Problems - Solving, Jase, Vol I.3, No. 2, 1972 . 
8.- Le système a une certaine garantie de continuité; il n'est pas éphémère et récupérera après une crise. (1)

Nous basant sur ce modele général et surtout sur ces caractéristiques, nous allons maintenant procéder à la vérification du modele conceptualisé.

Le systeme a comme objectif de regrouper les ateliers de débosselage dans le but de faire l'unanimité de ses membres autour de problèmes communs et d'en dégagér des actions communes, cohérentes, articulées et dans l'intérêt des membrés eux mêmes.

Il dispose de mesures de controle puisqu'il identifie les situations problématiques, pose des actions et controle si ces dernieres apportent des solutions aux problèmes.

Il se divise en quatre sous-systèmes ayant eux-mêmes les caractéristiques d'un système.

I. Prévost, Paul, L'organisation, un système d'activités humaines: Concept d'un modele général, octobre 1980 . 
Chaque composante du sous-système génère un flux d'information et le transmet à l'ensemble.

Il acquiert des ressources humaines et physiques à partir du système et de l'environnement.

Il répond à des besoins permanents exprimés par les propriétaires d'ateliers consultés, ce qui lui assure une certaine permanence.

La prise de décision est structurée et le mode de fonctionnement lui assure sa continuité.

$$
\text { 3.3- COMPARAISON }
$$

Nous allons dans cette section, comparer le modele proposé avec la situation que nous avons décrite au Chapitre II.

\subsection{1- Comparaison du modelle conceptuel avec la réalité}

La situation qui existe dans le domaine du débosselage présentement pénalise principalement les ateliers de débosselage indépendants. En effet, il est 
difficile pour eux de pouvoir communiquer et se faire entendre sur une base individuelle. A l'opposé, le modele propose une association de tous les ateliers afin d'avoir un interlocuteur unique et privilégié avec le reste des intervenants de $l^{\prime}$ industrie du débosselage.

Il en est de même pour les activités reliées au décret et ultimement pour l'obtention de politique d'achat de produits et services à meilleur prix avec des garanties d'approvisionnement.

Dans le passé, des propriétaires ont tenté de mettre sur pied un regroupement. Celui-ci était concentré à l'intérieur d'une ville et, dans certains cas, touchait d'autres entreprises de l'industrie automobile ( stations de services, ateliers de réparation etc...).

Ces expériences ont toujours avortées étant donné le peu de temps que les organisateurs y consacraient, l'absence d'assistance technique adéquate et le manque de clarté au niveau des structures à mettre en place. 
De plus, les promoteurs négligeaient de prévoir des activités de support propres à assurer la continuité nécessaire à tout regroupement une fois que l'aspect de nouveauté s'est estompé ou que le problème à la base de l'idée du regroupement s'est éliminé.

Ainsi donc, le modèle proposé présente un changement important avec la situation que vivent les ateliers de débosselage. Il présuppose une phase d'orientation très importante laquelle, si elle est bien accomplie, ne pourra que déboucher sur une structure adéquate, souhaitable, réaliste et performante. 
CHAP ITRE IV

IMPLANTATION 


\section{CHAPITRE IV}

\section{IMPLANTATION}

\section{1- LES ACTIVITES}

Les chapitres précédents ont permis d'approfondir la situation existante, d'identifier la problématique particulièrement celle des débosseleurs, de retenir le problème qui nous est apparu le plus important et de conceptualiser un système propice à apporter des solutions.

Nous sommes maintenant rendus à l'étape de la mise en application des solutions que nous avons décidées de privilégier. Nous nous proposons maintenant de vous présenter le travail qui a été effectué par le groupe de travail provisoire lequel a abouti à la mise sur pied d'une association visant à regrouper tous les ateliers de débosselage et qui porte le nom de: REGROUPEMENT DES CARROSSIERS DU SAGUENAY INC. 
Dans un premier temps, nous élaborerons sur les grandes lignes qui ont été envisagées pour démarrer le regroupement et surtout, par un mécanisme de support, assurer un intérêt suffisamment fort de la part des membres pour lui assurer un maximum de chance de survie.

Nous parlerons alors des lignes directrices et du mode de fonctionnement qui ont été approuvés par l'assemblée générale de formation particulièrement: les buts du regroupement, les pouvoirs de l'assemblée générale et du conseil d'administration, la description de tâches des principales fonctions, les conditions d'admission, l'administration courante du regroupement et la formation des premiers comités.

Dans un second temps, la démarche se poursuivra par l'élaboration des activités entourant ce lancement de même que toutes les actions qui ont résulté depuis.

Ces activités seront décrites par sous-sytèmes et selon l'ordre chronologique de réalisation. 


\section{2. -Lignes directrices et mode de fonctionnement du Regroupement des Carrossiers du Saguenay INC}

Dans cette section, nous decrirons les éléments constitutifs et la philosophie de base qui ont été favorisés lors de la création du regroupement. Elle reprend l'ensemble d'un document de travail que nous avions préparé en mai 1983 à l'intention du comité provisoire et de l'assemblée générale de formation, lequel document a été approuvé lors de l'assemblée de formation en juin 1983 .

\subsection{1- Buts du Regroupement}

Le Regroupement des Carrossiers du Saguenay Inc est né du besoin qu'ont exprimé les propiétaires d'ateliers de solutionner des problèmes pour lesquels ils n'ont pour ainsi dire aucun pouvoir individuellement.

Ainsi le Regroupement a pour but de faire l'unanimité de ses membres autour de problèmes communs et d'en dégager des actions cohérentes, articulées et dans l'intérêt des membres et de l'ensemble du secteur du débosselage. 
4.2.2- Pouvoirs de l'assemblée générale

Nonobstant le paragraphe précédent, les membres de l'association présents en assemblée générale auront les pouvoirs suivants:

1.- Elire un conseil d'administration une fois 1 'an;

2.- Mandater le C.A. pour qu'il entreprenne ou fasse entreprendre des actions précises;

3.- Appouver ou réfuter les décisions du C.A.;

4.- Etre souveraine quand à la détermination des grandes orientations du regroupement.

\section{2 .3 - Roles et pouvoirs du Conseil d'Administration}

Le conseil d'administration quant à lui, aura les obligations suivantes pour lui permettre un déroulement et un développement du regroupement:

$$
\text { 1.- assurer une continuite dans le }
$$

regroupement;

$$
\text { 2.- être responsable de l'application des lois }
$$
et règlements approuvés par l'assemblée générale; 
3.- prendre des actions prudentes en regard des objectifs du regroupement;

4.- autoriser les décisions financières et opérationnelles du regroupement;

5.- représenter le regroupement auprès des autres corps de la société;

6.- exécuter les décisions approuvées par l'assemblée;

7.- rendre compte de ses activités périodiquement auprès de l'assemblée générale;

8.- obtenir les informations pertinentes en ce qui concerne les activités couvertes par le regroupement;

9.- être responsable de l'usage approprié des ressources humaines, physiques et financières;

10.- entreprendre toute action qu'il juge appropriée pour exécuter le voeu ou les directives de l'assemblée et/ou pour préserver l'intérêt général de ses membres;

11.- s'adjoindre toute personne qu'il jugera apte à exécuter des actions précises et confier à celle-ci un mandat clair respectant le voeu, les directives ou l'intérêt de l'assemblée générale; 


$$
\text { 12.- accepter, refuser suspendre ou expulser }
$$
tout membre ou membre potentiel de l'association qui contreviendrait aux statuts et règlements de celle-ci en conformité avec l'article à cet effet.

\title{
4.2.4- Organigramme du Regroupement
}

\begin{abstract}
Nous vous présentons l'organigramme de l'association qui est en vigueur actuellement. Par la suite, nous exposerons dans la section 5 la description de tâches de chacune des composantes. (Voir SCHEMA NO 12)
\end{abstract}

4.2.5- Description de tâches

La présente section couvre la description des pricipales tâches qui seront dévolues à chacune des constituantes de l'organigramme présenté à la section précédente.

$4 \cdot 2 \cdot 5 \cdot 1-$ Membres

1.- assistent aux assemblées générales;

2.- élisent le conseil d'administration; 
SCHEMA NO 12

REGROUPEMENT DES CARROSSIERS DU SAGUENAY INC

DRGANIGRAMME

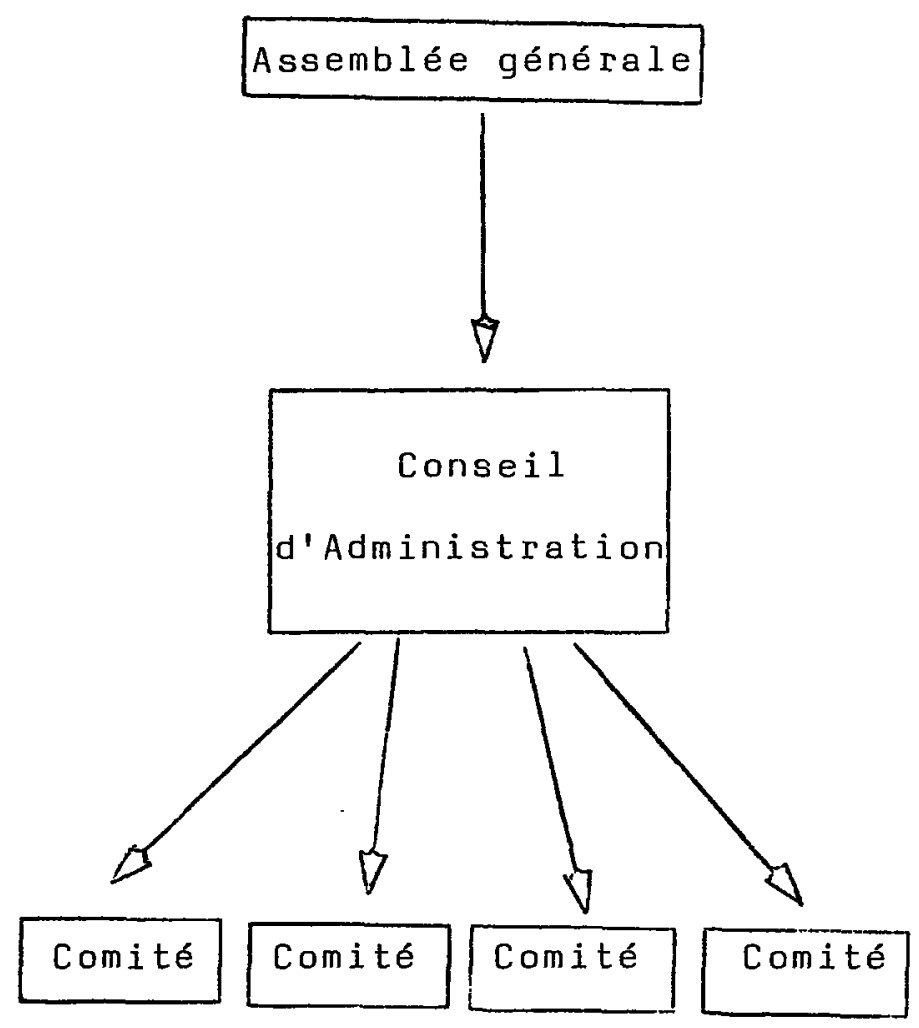




$$
\text { 3.- s'impliquent personnellement dans }
$$
l'application de solutions pratiques lorqu'ils sont sollicités;

4.- approuvent les décisions prises par le C.A. 5.- se plient aux résolutions prises majoritairement lors de $l$ 'assemblée générale;

6.- respectent les statuts et règlements de l'association;

7.- signalent tout manquement ou situations illégales dont ils ont connaissance au C.A. ou à un de ses représentants doment autorisé;

8.- participent aux comités formés par le C.A.; 9.- font la promotion du regroupement dans le milieu;

$$
\text { 10.-identifient, sollicitent et suggèrent }
$$
d'autres membres au C.A. . 


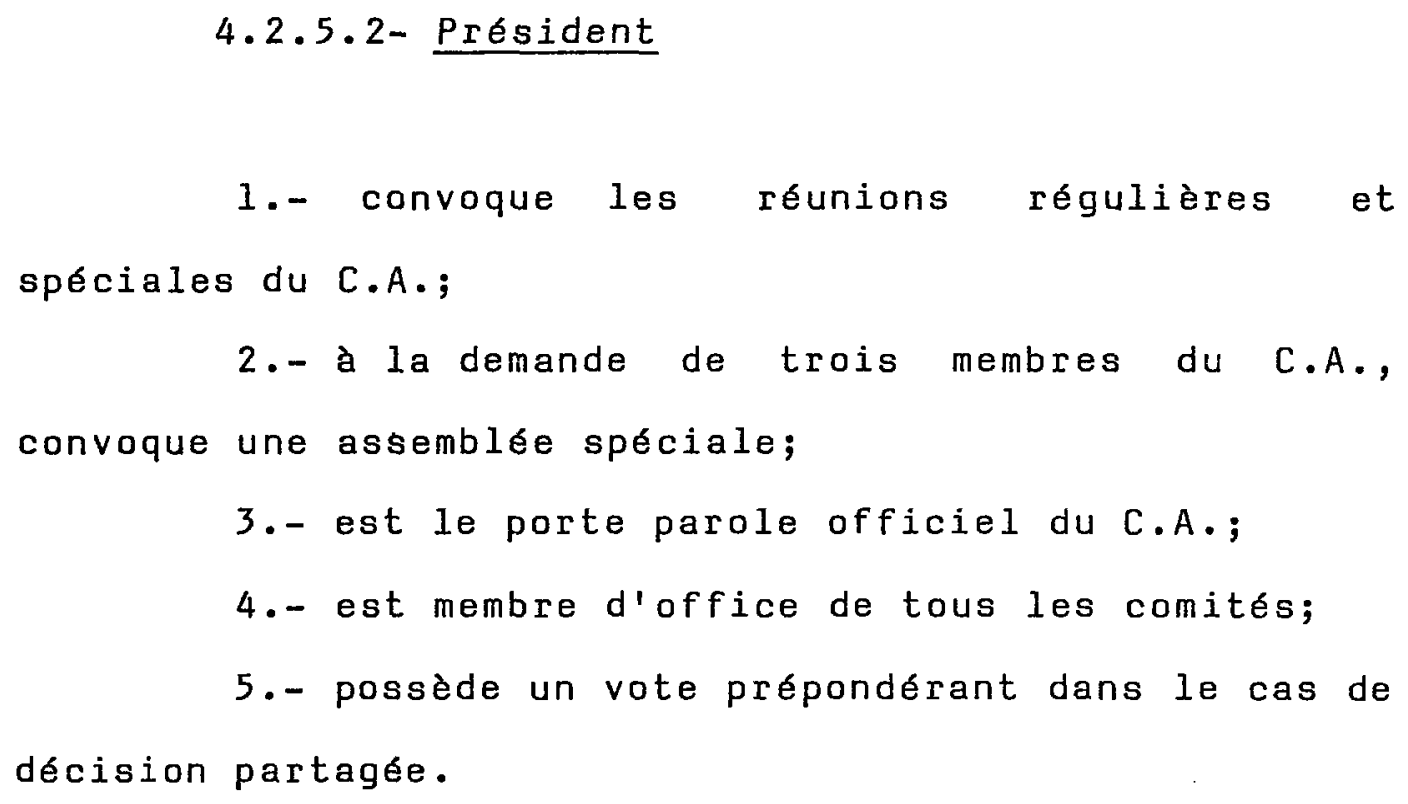


3.- est responsable de la mise à jour du livre des minutes de la compagnie;

4.- est le gardien du sceau de la compagnie;

5.- veille à produire des extraits des minutes pour diffusion selon le désir de l'assemblée générale ou du C.A.;

6.- prépare les ordres du jour de toutes les assemblées en accord avec le président;

7.- convoque au nom du président toutes les assemblées;

8.- vote sur les décisions du C.A. .

$4 \cdot 2 \cdot 5.5-$ Trésorier

1.- assiste aux réunions du C.A.;

2.- est responsable de la comptabilité de l'association;

3.- prépare les états financiers périodiques à la demande du C.A. ou de l'assemblée générale;

4.- tient à jour les livres de banques;

5.- approuve les dépenses de l'associaition;

6.- vote sur les décisions du C.A. . 


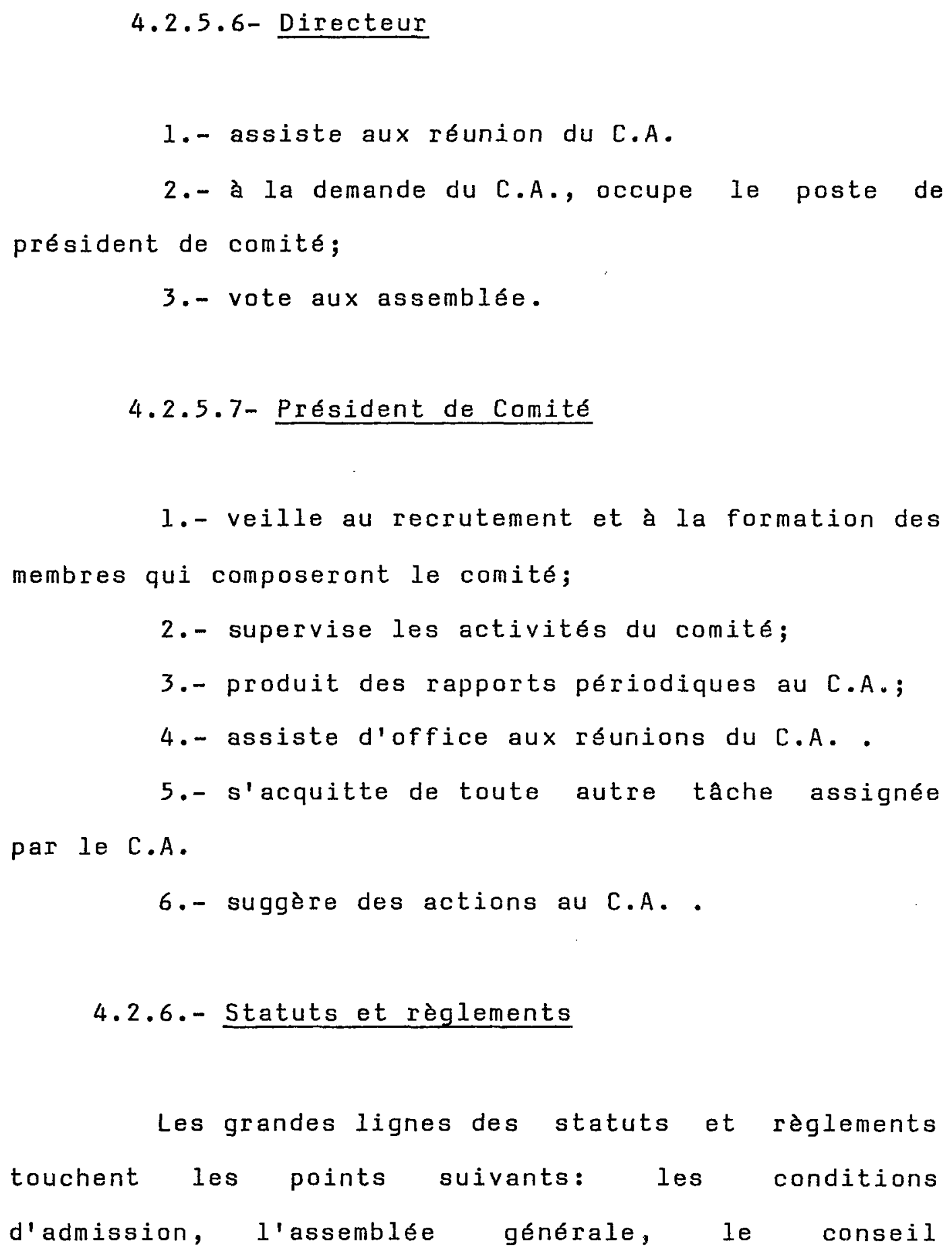


d'administration, l'administration générale, la cotisation, le comité de surveillance. Nous allons maintenant reprendre chacun de ces éléments plus en détails.

\subsubsection{1- Conditions d'admission}

Afin de faire partie du Regroupement des Carrossiers du Saguenay Inc, les futurs membres devront respecter les conditions suivantes:

1.- oeuvrer dans le domaine de la peinture et du débosselage;

2.- opérer un commerce de peinture et de débosselage en tant que propriétaire ou son représentant dâment autorisé;

3.- être enregistré auprès du comité paritaire de l'automobile, organisme responsable de l'application du décret régissant les activités du débosselage depuis le premier juin 1983;

4.- être considéré comme étant une personne ou une entreprise responsable, effectuant un travail professionnel; 
5.- répondre à toutes les exigences du décret cité plus haut;

6.- posséder toutes ses licences d'affaire permettant d'exercer ses activités;

7.- s'engager à respecter tous les règlements mis de l'avant par le C.A. et/ou l'assemblée générale;

8.- payer la cotisation annuelle requise.

Toute dérogation à ces conditions pourra entrainer l'expulsion de l'association et la perte des droits et privilèges ainsi que toute cotisation ou partie de cotisation déjà payée.

\subsubsection{2- L'assemblée générale}

Toutes les personnes ayant dament rencontré les conditions d'admission sont membres de l'assemblée générale du Regroupement des Carrosiers du Saguenay Inc.

1.- L'assemblée générale est responsable de déterminer et d'approuver les orientations générales de l'association;

2.- elle est responsable de l'élection des membres du C.A.; 
3.- durant les périodes de votation, la règle de un vote un membre prédomine et le vote majoritaire l'emportera;

4.- en cas d'égalité, le vote du président sera prépondérant;

5.- seuls les membres en règle auront le droit de vote;

6.- pour toutes les assemblées, le quorum sera fixé à quinze (15) actionnaires en règle.

7.- l'assemblée générale annuelle devra être convoquée dans les 60 jours suivant la date de cloture de l'exercice financier qui aura été choisie par l'assemblée des membres;

8.- la procédure de convocation et de déroulement des assemblées devra respecter le code de procédure des assemblées délibérantes (Code Morin);

9.- il appartiendra au président de canvoquer toute assemblée générale spéciale lorsqu'il le jugera à propos. 


\section{$4.2 .6 .3-\underline{\text { Le conseil d'administration }}$}

Le conseil d'administration devra être structuré selon l'organigramme et les tâches identifiées dans les sections précédentes.

1.- Les membres seront élus à l'assemblée générale annuelle doment convoquée à cette fin;

$$
\text { 2.- le conseil d'administraion devra }
$$

obligatoirement se réunir à quatre (4) reprises durant l'année en plus de l'assemblée générale annuelle;

3.- dans tous les cas d'assemblées, le quorum sera fixé à $50 \%$ des membres;

4.- le conseil d'administration sera soumis à toutes autres règles ou obligations telles que mentionnées dans les descriptions de tâches ou selon le désir de l'assemblée générale;

5.- il aura la responsabilité de déléguer des représentants sur tous les organismes pour lesquels la présence est requise et/ou pour l'intérét de l'association. 


\subsubsection{4- Administration}

1.- Les opérations financières quotidiennes seront confiées au trésorier lequel effectuera les transactions approuvées par le C.A.;

2.- les états financiers annuels devront être préparés par une firme d'experts comptables reconnue et acceptée par l'assemblée générale. Ces états financiers devront etre disponibles dans les 60 jours suivant la fin de l'exercice financier;

$$
\text { 3.- le trésorier verra à préparer des états. }
$$

financiers intérimaires régulièrement et en déposera une copie à tous les trois mois à une réunion du C.A.;

$$
\text { 4.- tous les livres comptables, registres, }
$$
livres des minutes ou comptes rendus des assemblées seront accessibles en tout temps par les membres en règle qui n'auront qu'a adresser une demande formelle au C.A.;

5.- le C.A. est responsable du respect des lois et règlements de l'association et aura a prendre les décisions qui s'imposent le cas échéant. 


\subsubsection{5- Cotisation}

1.- Pour faire partie du Regroupement des Carrossiers du Saguenay Inc, la cotisation sera fixée à $\$ 150.00$ annuellement. Toutefois, elle pourra être modifiée à tous les ans sur recommandation du C.A. et après approbation de l'assemblée générale;

2.- la cotisation annuelle constitue une prime d'adhésion et ne signifie pas la possibilité d'autres frais dans le cas d'activités spéciales ou particulières. Dans de tel cas, ces frais devront avoir été approuvés par l'assemblée générale.

\subsubsection{6- Comité de surveillance}

Pour protéger le Regroupement et ses membres, un comité disciplinaire aura la responsabilité de statuer sur les cas pouvant entrainer l'expulsion d'un membre et fera ses recommandations au C.A. qui prendra alors une décision. 
- Composition du comité

Le comité sera composé de quatres (4) membres dont trois ( 3 ) choisis par l'assemblée générale et un (1) par le C.A. .

\section{- Procédure de fonctionnement}

- Le comité devra se réunir aussi souvent que les situations l'exigent.

- Il aura à se pencher sur tous les cas qui lui auront été soulignés par les membres en règle.

- Il sera responsable d'analyser objectivement chaque cas et pourra convoquer toutes les personnes qu'il jugera nécessaire dans les circonstances.

- Ses recommandations devront etre exécutées par le C.A. à moins que des faits nouveaux ne permettent de trouver une nouvelle solution.

\section{- Catégories d'offenses}

Dans le but de préciser les offenses et leurs gravités, celles-ci seront classées par niveau en allant 


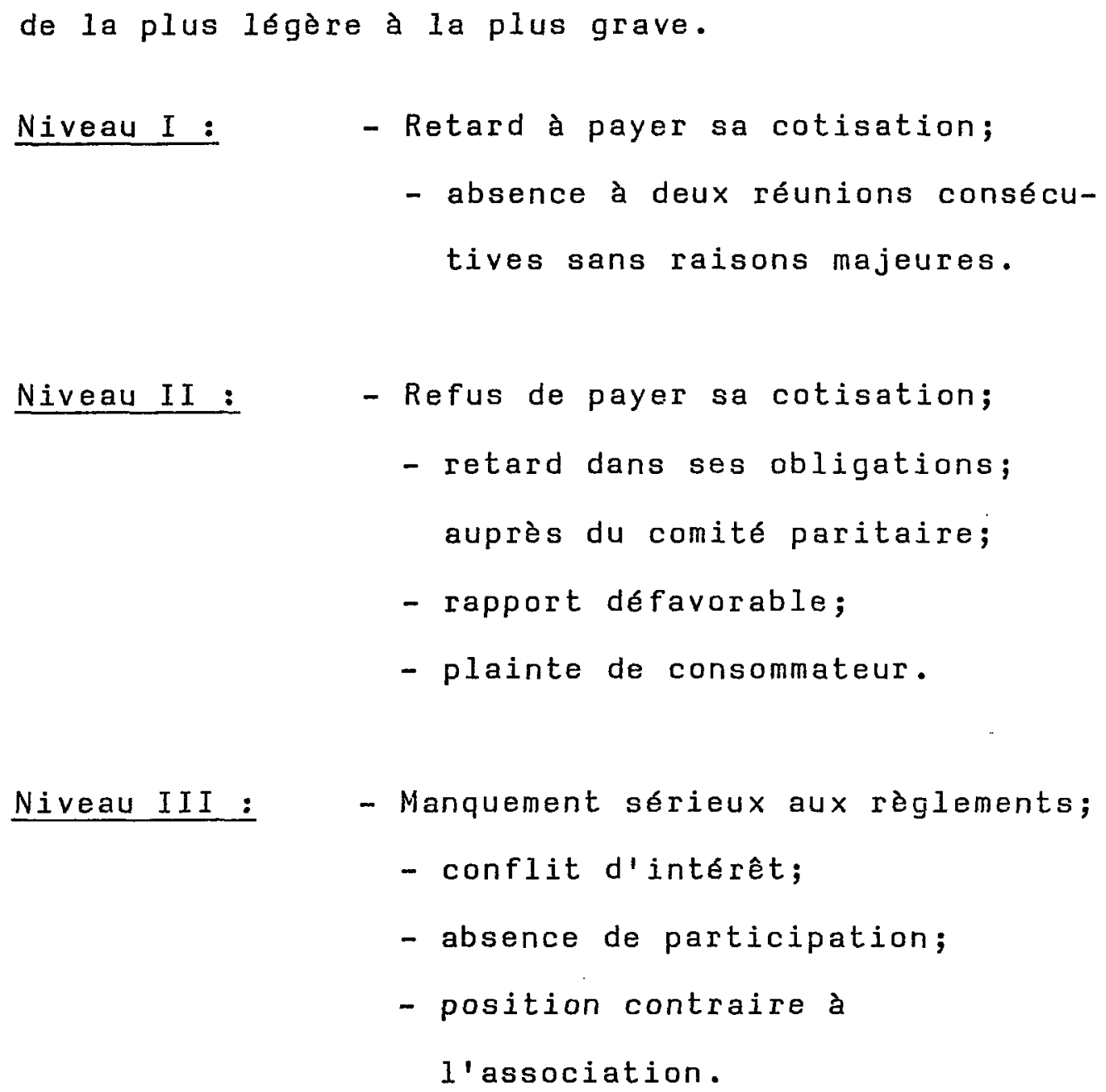

Niveau III : - Manquement sérieux aux règlements;

- conflit d'intérêt;

- absence de participation;

- position contraire à l'association.

\section{- Pénalité}

Nous incluons maintenant des possibilités de pénalités qui pourront être données aux membres fautifs. Niveau I : - Lettre d'avertissement. 


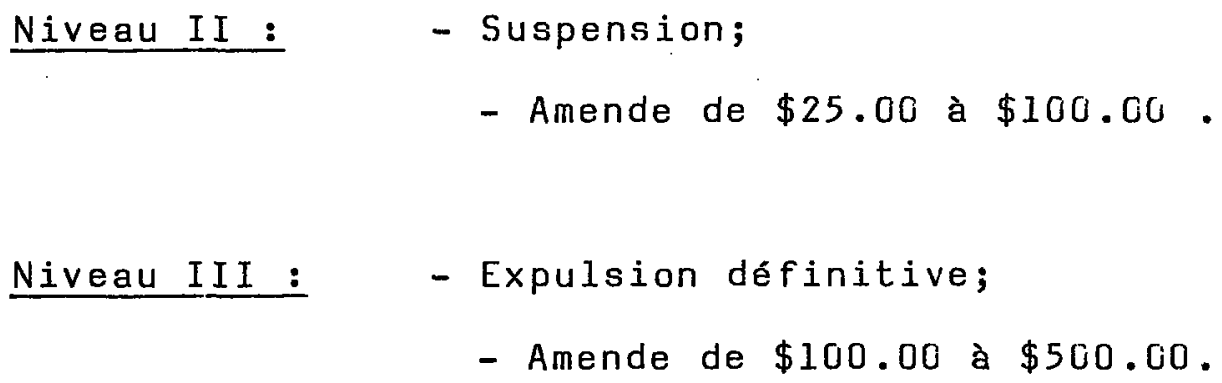

\section{- Admission subséquentes}

Passé soixante (60) jours après l'incorporation de la charte du regroupement, tout nouveau membre devra être accepté par le C.A. lors d'une séance régulière ou spéciale.

\section{- Mécanisme de sortie-expulsion}

Des raisons sérieuses peuvent amener l'expulsion ou la suspension d'un membre. Sans limiter le contenu de la présente, les raisons suivantes apparaissent comme étant les principales:

- décès d'un membre;

- conflit d'intérêt;

- insolvabilité, malversation, fraude;

- incompétence reconnue; 
- départ volontaire;

- refus de respecter les règlements.

Dans tous les cas, toute cotisation ou partie de cotisation est nullement remboursable.

\subsection{7.- Les comités}

Par expérience, nous savons qu'une association ne peut penser survivre si elle n'implique pas le maximum de membres. Aussi, pour éviter que les membres du C.A. soient perçus comme un groupe à part et pour faciliter le membership, le militantisme et la venue d'idées nouvelles en cours de mandat, nous favorisons grandement la formule des comités.

\subsubsection{1- Que sont les comités}

Les comités sont formés de membres choisis pour leur compétence respective à faire avancer un dossier précis. Ainsi, le C.A. aura à soumettre aux présidents des comités des mandats clairs qu'ils auront à mener à bien. 
Il reviendra au président du comité de nommer ses membres, de structurer son comité, de prévoir des réunions et de suggérer des actions au C.A. tout en bénificiant de tous les services disponibles pour le C.A. .

A noter que le président ou son représentant est membre d'office de tous les comités.

\subsubsection{2- Combien de comités}

A la limite, on pourrait souhaiter que tous les membres fassent partie d'au moins un comité. Mais, c'est sârement là un objectif à très long terme. Aussi, nous nous devons d'essayer d'identifier au départ, les membres les plus dynamiques et d'exploiter ce dynamisme.

Nous avons identifié au moins huit (8) comités qui pourraient aider le regroupement et le secteur du débosselage. La liste que nous en faisons est nullement exhaustive. 


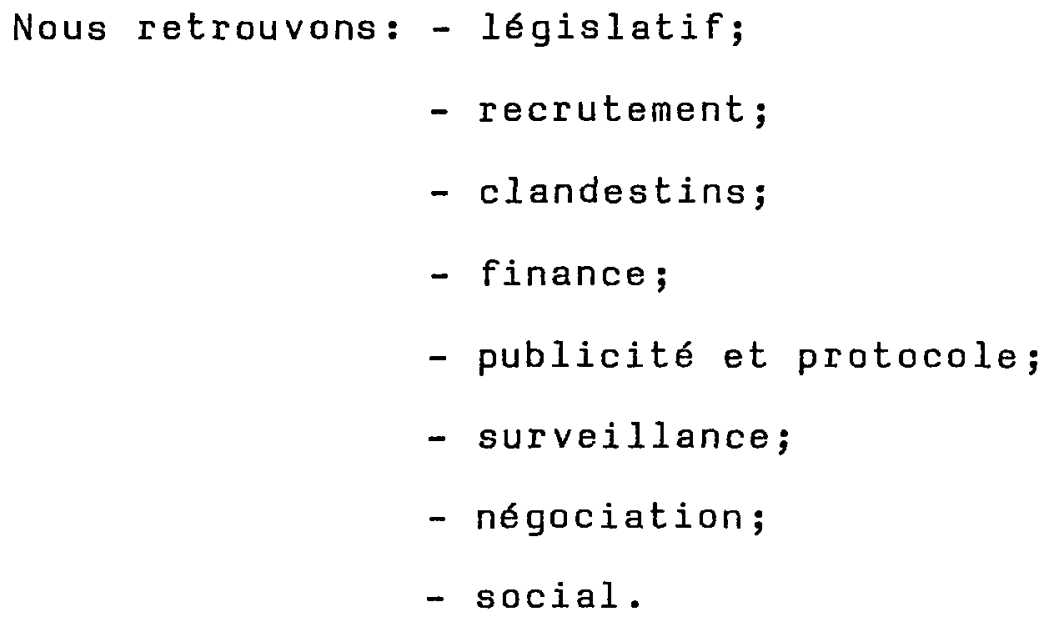

\section{$4 \cdot 2 \cdot 7 \cdot 3-$ Les priorités}

Nous avons pensé établir la priorité pour la formation des comités en fonction des problèmes soulevés par l'assemblée générale.

Pour assurer le démarrage et la survie du regroupement, nous croyons prioritaire la formation du comité de recrutement. Celui-ci aura à solliciter tous les membres possibles à l'intérieur du Saguenay.

Pour ce qui est du problème des ateliers clandestins, un autre comité devra être formé. Cependant, il est fondamental que le C.A. soit renseigné et documenté sur les différentes réglementations 
municipales et provinciales. Donc, en support du comité des clandestins un autre sera mis sur pied, le comité législatif.

Un autre aspect que nous n'avons pas voulu négliger, concerne la gestion financière de l'association. Aussi avons-nous dès le départ procédé à la formation d'un comité de finance lequel est constitué de trois membres en plus du trésorier. Il a comme priorité de s'assurer d'une saine gestion des fonds confiés par les membres et doit rendre compte périodiquement de cet aspect au conseil d'administration ou à l'asṣemblée générale. Nous avons voulu ainsi éviter que des irrégularités puissent se produire et ce même si notre charte est dite à buts non lucratifs.

Les autres comités seront formés selon l'émergence de situations nouvelles. 
4.3- RESUME DES ACTIVITES PAR SOUS-SYSTEMES

\begin{abstract}
Nous incluons maintenant la liste des principales activités qui ont été réalisées depuis le début des travaux du comité provisoire en mars 1983. Ces activités sont regroupées selon les différents sous-systèmes retenus dans notre démarche. A l'exception de certaines activités du sous-système opérationnalisation, toutes les autres ont été réalisées selon le calendrier prévu au départ.
\end{abstract}

\title{
4.3.1- SOUS-SYSTEME ORIENTATION
}

Cette première étape qui s'est déroulée entre le mois de mars et le début de juin 1983 a été réalisée par le groupe de travail provisoire mis sur pied initialement par l'Association PME et continué avec la participation la Mâtrise en Gestion des PMO de l'UQAC. Trois réunions eurent lieu durant cette période.

\section{$4.3 .1 .1-$ Liste des activités}

1.- Analyser la problématique commune.

1.- Identifier les problèmes connus;

2.- Identifier les problèmes potentiels;

3.- Recueillir l'information. 
2.- Déterminer les objectifs initiaux.

1.- Marier les objectifs à la problématique.

3.- Evaluer la pertinence d'actions communes.

1.- Examiner les actions individuelles;

2.- Evaluer leurs résultats.

4.- Analyser la pertinence de se regrouper.

1.- Cueillette d'information;

2.- Recherche d'associations existantes.

5.- Envisager structure d'accueil provisoire.

1.- Identifier personnes intéressées;

2.- Identifier la forme pertinente;

3.- Obtenir support extérieur.

\subsection{2- SOUS-SYSTEME STRUCTURATION}

Les activités propres à ce deuxième sous-système furent réalisées durant le mois de mai 1983. Les étapes ont encore une fois été accomplies par le comité provisoire. Afin de bien cerner l'état d'avancement, nous avions présenté à ce moment, un rapport d'étape schématisé lequel est fourni. ( Schéma No. 13) 


\section{SCHEMA NO 13}

RAPPORT D'ETAPE DES TRAVAUX DU REGROUPEMENT DES CARROSSIERS DU SAGUENAY
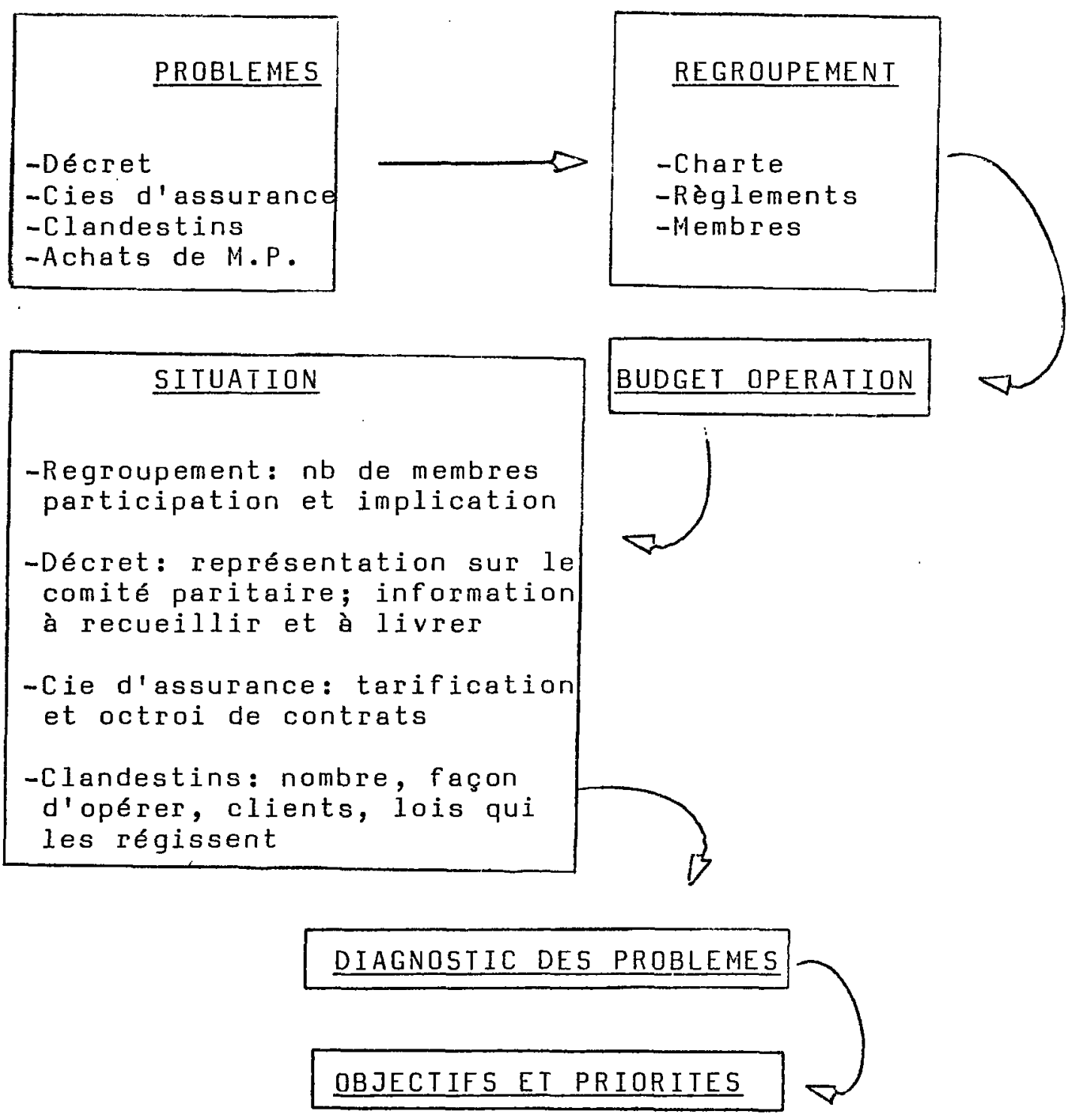
4.3.2.1- Liste des activités

1.- Etablir structure d'accueil provisoire.

1.- Rassembler les membres;

2.- Contacter personnes ressources;

3.- Choisir un responsable;

4.- Choisir un secrétaire de réunion.

2.- Préciser les objectifs et les orientations.

1.- Revoir les problèmes identifiés;

2.- Consulter les intervenants;

3.- Décider des objectifs;

4.- Décider des orientations.

3.- Reviser les études et informations.

1.- Consulter les débosseleurs;

2.- Contacter le comité paritaire;

3.- Compiler les informations.

4.- Evaluer l'impact du regroupement.

1.- Evaluer l'impact politique;

2.- Evaluer l'impact économique;

3.- Evaluer l'impact opérationnel. 
5.- Estimer les services potentiels à offrir. 1.- Envisager les achats regroupés;

2.- Envisager les conseils légaux;

3.- Envisager les autres services.

6.- Estimer les coots.

1.- Budgétiser les activités;

2.- Estimer la cotisation;

3.- Etablir budget provisoire. 
Les étapes à l'intérieur de ce sous-système ont été amorçées en mai 1983 et se sont poursuivies jusqu'à la fin de juin 1983. Le point important fat sans conteste l'assemblée générale de formation qui s'est déroulée le 31 mai 1983. Stratégiquement, il s'agissait d'une date excellente puisque le nouveau décret régissant les activités des débosseleurs entrait en vigeur le lendemain et que plusieurs, si ce n'est la majorité, ignoraient cet état de fait. Nous avons donc orienté notre publicité autour de ce thème et ainsi, un peu plus d'une vingtaine d'ateliers y était représentés.

Devant l'intérêt demontré à cette occasion, une seconde assemblée générale eut lieu le 7 juin 1983 pour donner de l'information aux membres sur le sujet et pour procéder à l'adoption des statuts et règlements. 


\subsubsection{1- Liste des activités}

1.- Convoquer l'assemblée générale.

1.- Préparer l'assemblée;

2.- Expédier une lettre à tous les ateliers;

3.- Effectuer un contact téléphonique;

4.- Réserver les locaux;

5.- Diriger la réunion.

2.- Elire le conseil d'administration.

1.- Choisir un président d'élection;

2.- Présenter les modalités d'élection;

3.- Procéder aux mises en nomination;

4.- Elire le conseil.

3.- Adopter statuts et règlements.

1.- Reviser la littérature;

2.- Préparer un document de travail;

3.- Faire approuver le document par le CA;

4.- Présenter document à l'assemblée générale;

5.- Faire approuver document par l'assemblée;

6.- Préparer la charte;

7.- Faire approuver le nom. 
4.- Poursuivre le recrutement.

1.- Former le comité de recrutement;

2.- Préparer le matériel de recrutement;

3.- Expédier le matériel de recrutement;

4.- Assurer le suivi;

5.- Embaucher un permanent au recrutement.

5.- Etablir les priorités à entreprendre.

1.- Suggérer des actions précises;

2.- Faire approuver les actions. 


\subsection{5- SDUS-SYSTEME RECRUTEMENT}

Une des étapes les plus importantes dans un recrutement comme celui que nous avons mis sur pied se réfère au recrutement des membres. Ceci fut d'autant plus difficile que nous n'avions pas de produit concret à offrir surtout au début de l'exercice.

En effet, nous ne pouvions pas parler d'offrir des escomptes sur des achats de produits ou services puisque cela n'avait pas été considéré prioritaire par les membres fondateurs. Nous ne pouvions davantage parler d'actions concrètes au niveau du décret puisque, tant que nous n'avions pas de structure légale, il était impossible de pouvoir se faire entendre. Notre produit se situait au niveau des idées et il n'y avait rien de tangible à considérer.

$$
\text { Egalement, parmi les propriétaires qui }
$$

assistèrent aux assemblées de fondation, ils s'en trouvaient plusieurs qui avaient tenté dans la passé de créer des regroupement et qui avaient échoué. Ils étaient donc un peu amers et disaient à qui voulaient 
Nonobstant ces faits, nous avons orienté nos activités en fonction de ces points et avons mis l'emphase sur le décret et les aspects négatifs de ce dernier. A ceux qui nous demandaient comment il se faisait que le gouvernemnt avait décidé de régir leurs activités au moyen d'un décret sans qu'ils le sachent, nous leurs expliquions que des consultations publiques avaient eu lieu en novembre 1982 et, qu'étant donné l'absence d'un regroupement, personne n'avait cru nécessaire de faire opposition.

Nous avons démontré la nécessité d'apporter des modifications au décret et que la seule manière de le faire était de se constituer en association. Cette nécessité nous fat d'ailleurs confirmée dans une lettre que nous faisait parvenir le cabinet du Ministre du Travail. Cette lettre est founie en annexe.

Nous allons vous présenter le sommaire de nos actions. 


\subsubsection{1- Liste des activités.}

1.- Etablir plan de recrutement.

1.- Identifier les arguments favorables;

2.- Identifier les arguments défavorables.

2.- Préparer matériel de recrutement.

1.- Préparer lettre de sollicitation.

3.- Mettre sur pied le comité de recrutement.

1.- Trouver des membres dynamiques;

2.- Réunir le comité avec le CA;

3.- Planifier les opérations;

4.- Monter le contrat d'adhésion.

4.- Effectuer la campagne de recrutement.

1.- Procéder par ville;

2.- Envoi d'une lettrede sollicitation;

3.- Contacter personnellement les membres;

4.- Superviser les activités avec le CA.

5.- Enregistrer les nouveaux membres.

6.- Planifier les assemblées générales. 


\subsection{6- SOUS-SYSTEME OPERATIONNALISATION}

Le dernier sous-système a débuté officiellement suite à la seconde assemblée générale de formation le 7 juin 1983. A partir de ce moment, l'exécutif élu a entamé les démarches sur les problèmes soulevés. II s'est aussi engagé dans la phase de consolidation de la structure et dans la diffusion de son existence auprès des intervenants particulièrement, le ministère du travail, le comité paritaire de l'automobile, le centre d'estimation et les fournisseurs de pièces automibiles.

\subsubsection{1- Liste des activités}

1.- Entreprendre les actions retenues.

1.- Communiquer avec le comité paritaire;

2.- Communiquer avec le ministère du travail;

3.- Communiquer avec l'oppostion officielle;

4.- Communiquer avec les fournisseurs.

2.- Administrer le regroupement.

1.- Obtenir une charte provinciale;

2.- Ouvrir compte de banque;

3.- Percevoir les cotisations; 
4.- Etablir réseau de communication.

3.- Planifier les activités futures.

1.- Former le comité législatif;

2.- Former le comité sur les clandestins;

3.- Former le comité de négociation;

4.- Monter un questionnaire sur les achats;

5.- Monter questionnaire sur les clandestins;

6.- Préparer le code d'éthique.

4.- Publiciser le regroupement.

1.- Préparer une conférence de presse;

2.- Effectuer la conférence de presse;

3.- Préparer les pièces d'identification;

4.- Rencontrer les autres associations.

5.- Poursuivre le recrutement.

6.- Revoir les objectifs généraux.

1.- Assurer la cohérence des actions posées;

2.- Modifier les actions au besoin;

3.- Envisager le regroupement d'achat. 
7.- Assurer la survie de l'association.

1.- Imaginer des activités de support;

2.- Recueillir des informations;

3.- Mettre sur pied les activités.

4.4- COMMENTAIRES

Les activités qui ont été effectuées au cours de ce regroupement selon les sous-systèmes que nous avons identifiés et que nous venons de vous présenter dans ce chapitre, ont nécessité la préparation de plusieurs documents. Ceux-ci n'ont pas été intercalés dans les explications des différents sous-systèmes dans le but de ne pas surcharger inutilement le texte. Ces derniers sont donc présentés dans les annexes suivantes:

ANNEXE 1 : Le contrat d'adhésion

ANNEXE 2 : Le code d'éthique

ANNEXE 3 : Questionnaire sur les achats

ANNEXE 4 : Questionnaire sur les clandestins 
Par ailleurs, nous voulons ajouter que le regroupement a mis sur pied les activités de support suivantes dans le but d'assurer la survie de l'association, de faciliter le recrutement de nouveaux membres et pour maintenir un bon niveau de communication. Nous retrouvons:

- Ia mise sur pied d'un bulletin mensuel d'information pour tous les ateliers de débosselage de la région;

- la planification de cliniques de perfectionnement sur les nouveaux produits;

- la préparation de cours de formation sur les aspects techniques du débosselage;

- la préparation de cours de formation en gestion et administration de petites entreprises. 
CHAP I TRE V

CONTROLE 


\section{CHAPITRE V}

\section{CONTROLE}

\section{1- NECESSITE DU CONTROLE}

Afin de permettre au Regroupment des Carrossiers du Saguenay Inc de pouvoir progresser et de s'attaquer à tous les problèmes auxquels sont confrontés les ateliers de débosselage et, dans le but de compléter la démarche entreprise, il a été nécessaire de prévoir des activités ou des moyens de contrôle. C'est une démarche essentielle qui permet à tous les intervenants de pouvoir porter un jugement sur le déroulement des activités et d'y apporter les correctifs qui pourraient être jugés nécessaires.

Nous traiterons donc cet aspect sous deux volets soient: le volet interne, propre aux activités à l'intérieur du regroupement, et le volet externe, propre aux interrelations du regroupement avec son environnement particulièrement, les autres partenaires de l'industrie du débosselage et l'intervenant responsable de cette démarche. 
5.2- LE VOLET INTERNE

Ce volet touche, comme nous l'avons dit précédemment, les activités internes du regroupement et est relié aux sous-systèmes que nous avons identifiés.

\section{2 .1 - Le sous-système orientation}

Au niveau de ce sous-système, le contrôle est effectué par le conseil d'administration sur une base mensuelle lors de ses réunions régulières. A cette occasion, celui-ci evalue la pertinence des orientations qui ont été prises. Cela s'effectue avec la collaboration des présidents de comité qui assistent à ces réunions et qui apportent le poul des membres.

Si des modifications sont requises, elles sont discutées et la priorité des actions entreprises ou à entreprendre est alors modifiée.

\subsubsection{Le sous-système structuration}

Encore là, c'est au conseil d'administration, par l'entremise des différents comités mis sur pied, que revient la tâche d'effectuer les contrôles requis. 
De plus, nous vous reférons à la section 4.2 portant sur les statuts et règlements où il est question des différents comités mis sur pied ou à être formés pour ajuster le regroupement en conséquence.

\subsection{3- Le sous-système mode de fonctionnement}

Pour préserver l'intérêt général des membres, les statuts et règlements sont très explicites sur le mode fonctionnement. Un de ses traits caractristiques réside dans la transparence que nous avons voulu donner au regroupement. Il appartient donc à tous les membres de contrôler cet aspect.

Particulièrement, nous voulons parler du contrôle financier qui est assuré par un comité de trois personnes. Il y a aussi le comité de discipline qui, par ses pouvoirs, permet d'assurer un déroulement harmomieux des activités. 
$5.2 .4-$ Le sous-système recrutement

Le controle de ce secteur s'effectue par le conseil d'administration pour ce qui est du travail de recrutement proprement dit et nous incluons ici l'aspect sollicitation et planification des campagnes. Pour les problèmes d'admissibilité, c'est le comité de discipline qui verra à investiguer ce domaine.

\subsection{5- Le sous-système opérationnalisation}

Le contrôle opérationnel est sous la responsabilité du conseil d'administration avec la participation du consultant. Il touche toutes les phases de déroulement des activités et les décisions, actions, commentaires sont consignés dans les procès-verbaux des réunions. Ces documents sont disponibles sur demande.

\section{$5.3-$ LE VOLET EXTERNE}

Dans cette section, nous allons toucher les moyens que s'est donné ou qu'entend se donner le regroupement afin de pouvoir controler son environnement. Actuellement nous parlons de points de 
contrôle au niveau du décret, des ateliers clandestins et des fournisseurs.

\subsection{1- Le décret}

Tel que mentionné dans la Loi sur les Décrets de Convention Collective, l'administration est confiée à un comité paritaire regroupant en nombre égal les propriétaires et les employés.

Nous avons donc entrepris les démarches auprès du Ministre du Travail afin de devenir partie contractante au décret et pour pouvoir siéger sur le comité paritaire de l'automobile du Saguenay Lac St-Jean Inc. Ce dossier est mené rondement et, prenant en considération les informations que nous avons reçues dernièrement du cabinet du Ministre, nous n'entrevoyons pas de difficultés à être accepté.

\section{$5.3 .2-$ Les assureurs}

Afin d'améliorer la position des ateliers face aux compagnies d'assurance, l'association se devra d'entreprendre des actions collectives pour sensibiliser ce partenaire aux difficultés rencontrés et pour 
négocier des améliorations aux tarifs en vigeur. A cet effet, lors de la dernière assemblée générale, il a été accepté par tous de majorer le tarif de \$2.00 l'heure. Suite à cette réunion, un représentant de l'association a visité tous les ateliers de la région lesquels ont unanimement accepté la résolution et ont signé le formulaire requis afin que nous puissions aviser le Groupement des Assureurs du Québec. Ceci constitue un précédent dans l'industrie au Saguenay et témoigne de la nécessité de l'association pour les négociations futures.

\section{$5.3 .3-$ Les ateliers clandestins}

Ce problème capital pour les débosseleurs, est suivi avec attention par le conseil d'administration. Tel que présenté à l'ANNEXE 4, nous avons réalisé un questionnaire sur le sujet. Il a été distribué à tous les ateliers indépendants de la région pour aider l'exécutif, lequel est à monter un dossier qui sera soumis au comité paritaire dès que le regroupement aura reçu son accréditation.

En effet, il semblerait qu'il est dans les intentions du comité paritaire d'entreprendre des 
actions concrètes pour diminuer l'impact de ces ateliers. Il est donc dans l'intérét de l'exécutif d'aider les inspecteurs en leur fournissant le plus d'information possible sur ce sujet.

\section{$5.3 .4-$ Les fournisseurs}

Depuis quelques temps et, même si au départ ce domaine n'était pas une priorité, le regroupement a noté que certains fournisseurs en particulier les concessionnaires automobiles, avait commencé à modifier unilatéralement les conditions de paiement sur les achats de pièces. Devant cette situation, le comité des achats a tenté de changer l'attitude des concessionnaires du Saguenay. Comme il ne semblait pas $y$ avoir d'entente possible, il a obtenu de concessionnaires du Lac st-Jean des conditions identiques à ce qu'avaient les ateliers au préalable. Cela a permis à tous les membres de jouir d'un avantage qu'ils n'auraient pas pu avoir individuellement. 
CHAPITRE VI

REFLEXION METHODOLOGIQUE 


\section{LE RAPPORT ACCADEMIQUE}

\section{1- REFLEXION METHODOLOGIQUE}

$6.1 .1-\underline{\text { La méthode }}$

Au cours de l'apprentissage qui s'achève, la
démarche utilisée a été celle dite du
diagnostic-intervention associé au diagnostic
organisationnel et à la recherche-action.

La recherche-action est avant tout un processus d'apprentissage. Associée à la méthodologie de Peter B. Checkland, elle vise à rendre les intervenants autonomes en leur permettant de diagnostiquer les problèmes prioritaires et à y apporter des solutions concretes. La recherche-action vise donc l'expérience de formation dans l'action. Pour se faire, la relation entre chercheurs et acteurs doit nécessairement en être une de collaboration, les personnes impliqués cheminant ensemble dans un processus d'apprentissage mutuel. 
Comme le mentionnait Monsieur Gérald Fortin

lors du colloque sur la recherche-action tenu en octobre 1981 à l'Université du Québec à Chicoutimi:

Le sens véritable de
recherche-action la
recherche d'une solution pour le
groupe où le chercheur milite.
Ca pose des problèmes entre la
thérie et la simplicité du
résultat, entre l'apprentisage
compliqué et la nécessité d'arriver
à une solution rapidement. Le
chercheur n'est pas nécessairement
militant mais il est sympathisant.
Ilest accepté comme sympathisant
par le groupe et celui-ci lui
demande de faire de la recherche. Ce
qu'il faut voir là dedans, c'est une
sorte d'intervention où le chercheur
joue un rôle très actif. (6)

Nous retenons donc de cette démarche, outre l'aspect méthodologique, la nécessité de faire cheminer celle-ci conjointement par le chercheur et les acteurs de la façon la plus harmonieuse et la plus rapide possible. Cela implique une acceptation réciproque des intervenants dès le début.

6- Fortin, Gérald, Actes du colloque Recherche-Action, UQAC, $1981, \mathrm{p} .7$ 
Dans mon cas, cet aspect s'est effectué sans heurts de telle sorte, qu'après un mois de travail en commun, il nous a été facile d'établir un dialogue nous permettant de progresser dans la résolution du contenu problématique.

\section{$6.1 .2-\underline{\text { La démarche }}$}

La méthodologie que nous avons favorisée établi donc un processus inductif et déductif propre à la détermination d'une image juste de la situation pour en arriver à déterminer des éléments de solutions. Elle liste en plus, les principales activités que nous avons à réaliser et suggère un ordre séquentiel de ces mêmes activités.

Quoique cela n'ait pas causé de difficultés majeures, il nous est arrivé à certaines accasions, des problèmes de compréhension et d'agencement, puisque nous avions tendance à confondre la problématique existante et l'ancrage proprement dit. Ce phénomène provient à mon avis, du fait que nous nous faisions souvent dès le départ, une certaine idée de la situation et que nous envisagions alors des hypothèse de solutions. Si cela 
peut se vérifier dans plusieurs cas, il n'est pas certain que cela puisse se confirmer en tout temps.

Mais, comme notre première activité avait été de présenter aux acteurs la démarche que nous voulions favoriser, il était alors facile de se retrouver et de reprendre la séquence.

Pour le reste, nous n'élaborerons pas davantage sur le sujet compte tenu que les premières parties de ce document reprennent en détails tous les autres éléments de la démarche.

\section{2-LES CONNAISSANCES ACQUISES}

\subsection{1-Sur le contenu problématique}

L'expérience qui s'achève du point de vie accadémique (pratiquement, je suis encore impliqué dans le regroupement) m'a permis de prendre contact avec un secteur d'activité économique qui est pour le moins négligé par tous les intervenants socio-économiques de la région et de tout le Québec. 
En fait, tel que je le mentionnais dans l'introduction, il est surprenant de constater le peu d'appuis techniques, humains et financiers que peuvent recevoir les débosseleurs actuellement. Tous les programmes d'aides mis de l'avant par les différents paliers de gouvernement et il sont nombreux, ne sont jamais accessibles à cette catégorie.

Le problème proviendrait semble-t-il, du fait que cette industrie ne se situe ni dans le secteur tertiaire, ni dans le secteur secondaire. Elle se trouverait à la limite de ces deux classifications. Donc, si un programme pour l'industrie manufacturière est lancé, on nous dit que nous ne sommes pas éligibles parce que nous nous situons un peu plus dans le secteur service. Et l'inverse est également vrai.

Etant laissés pour compte, il en résulte que la littérature privée ou gouvernementale disponible est très mince pour ne pas dire inexistante. Le chercheur se doit de faire ses classes avec les débosseleurs eux-mêmes. C'est une expérience enrichisante il est vrai; mais parfois difficile particulièrement lorsque vient le moment de contrôler les résultats obtenus. 
Je me suis donc familiarisé avec tous les rouages de l'industrie: du processus de réalisation des travaux jusqu'au système global de gestion et de transformation. Depuis un an, jiai pu effectuer mon apprentissage du domaine avec un groupe représentatif et dynamique. J'ai surtout constaté comment ces individus se sentent démunis face à des problèmes comme ceux que nous avons relevés et que, sans support, ils ont tendance à se replier et se laisser dominer par la situation.

\subsection{2-Sur les regroupements d'entreprises}

Le phénomène des regroupements d'entreprises quoique très ancien, surtout dans la moyenne et la grande entreprise, a connu un essor important depuis quelques années. Il semble que le démarrage a débuté avec la crise économique; les entreprises tentant de se regrouper pour abaisser les cauts d'exploitation. Il y eut le Ministère de l'Industrie, Commerce et Tourisme qui a grandement contribué à la reprise de cette activité. 
En effet, ce ministère compte maintenant dans ses rangs des experts qui se déplacent dans les régions afin d'assister ceux qui sont intéressés à se regrouper. Malheureusement pour le secteur du débosselage, deux point précis viennent restreindre considérablement la portée de cette collaboration.

Il y a tout d'abord la situation que nous décrivions dans la section précédente soit: que les débosseleurs sont mal positionnés par rapport à la classification industrielle actuellement en vigueur. Ainsi, les experts concentrent leurs énergies principalement dans le domaine du regroupement des entreprises de détails ou de services et, à titre d'exemple, nous mentionnerons simplement le travail actuellement effectué pour assurer le démarrage des SIDAC (Société de développement des artères commerciales). Ils s'intéressent aussi aux regroupements d'entreprises manufacturières désireuses de se constituer en associations pour l'achat regroupé de matières premières. Donc, les débosseleurs ne sont pas compris dans ces deux catégories. 
Enfin, il y a le fait que les regroupements se réalisent généralement, comme nous venons de le dire, pour abaisser les coots d'opérations tels que la réduction de la facture pour l'achat des matiéres premières. Pour les promoteurs, il est alors facile de pouvoir démontrer de manière concrete les économies qu'une entreprise pourrait réaliser en se regroupant. Or, dans le débosselage, la proportion des dépenses reliées aux achats est négligeable par rapport à la rémunération (moins de $15 \%$ ). Nous ne pouvons alors jouer cette carte et offrir rapidement quelque chose de tangible.

Il devient alors difficile diutiliser la littérature offerte par le MICT ou ses représentants pour nous aider dans notre démarche car, celle-ci tourne presqu'exclusivement autour de cette hypothèse. D'ailleurs, ces personnes ont une disponibilité restreinte après etre intervenus dans les deux autres secteurs précités. Il nous fallait alors prévoir des activités de remplacement et assurer un suivi constant auprès de tous les membres potentiels pour conserver un intérêt suffisamment fort. 


\title{
6.2 .3 -Sur la démarche
}

\begin{abstract}
Concernant notre réflexion sur la démarche utilisée, nous voudrions mentionner les commentaires suivants. En effet, nous pensons qu'elle possède plusieurs avantages qui ne sont pas négligeables particulièrenment dans le cas de problèmes complexes et peu structurés tels ceux que nous avions à solutionner.
\end{abstract}

Elle permet de vérifier si notre vision de la situation problématique est suffisamment claire et précise. Ceci s'effectue par l'investigation complète de la question.

Elle est méthodique, rationnelle, souple et ne peut que nous faire déboucher sur une perspective raisonnable. Même s'il nous est arrivé de confondre certaines étapes, elle permet de nous retrouver facilement et de corriger un égarement temporaire.

Pour terminer cette partie, nous voudrions citer un extrait d'un document de M. Paul Prévost lequel résume très bien notre opinion: 


\begin{abstract}
Sa démarche est avant tout systémique mais avec un souci constant d'éviter deux éceuils de taille: d'une part, celui d'utiliser une terminologie à ce point vague que son utilité opérationnelle soit sacrifiée au dépens d'une vision globalisante devenue triviale; d'autre part, celui de subir la rigidité déformante d'un langage trop précis par rapport à la richesse de la situation étudiée. Contrairement à beaucoup d'autres méthodes qui'ne nous spécifient pas de règle opérationnelle de simplification du réel perçu, celle de checkland le fait en faisant ressortir la nécessité de choisir un point d'ancrage, une perspective, un angle particulier et privilégié pour l'examen d'une situation dont la richesse déborde nos capacités de modélisation. Parce que cette perspective privilégiée doit être sélectionnée par les intervenants eux-mêmes, elle constitue une règle pratique et non arbitraire de réduction pertinente de variétés. (7)
\end{abstract}

7- Prévost, Paul, Le diagnostic-intervention: une approche systémique au diagnostic organisationnel et à la recherche-action, LEER, 1983, pp. 34-35. 


\subsection{4-Pour le chercheur}

En ce qui me concerne, l'intervention réalisée dans le cadre de ce mémoire fut très enrichissante non seulement du point de vue thérique (ce que j'ai déjà mentionné dans ce chapitre), mais surtout, au niveau de toute la dimension humaine à l'intérieur de toute organisation.

Premièrement, on a qu'à penser comment il peut être difficile de faire se rencontrer des compétiteurs, de leurs demander de s'associer et de dévoiler une partie de leurs expériences et de leurs connaissances. Il y a la tout un jeu de diplomatie et de négociation pour que chacun accepte de s'ouvrir et contribue a la création d'un climat propice au dialogue.

Deuxièmement, la motivation qui anime les participants n'est pas toujours celle que l'on désire et, a ce moment, il nous faut penser constamment à des mécanismes et des activités qui soient assez forts pour maintenir un niveau d'intérêt minimum. 
Nous retrouvons aussi toute la notion du sentiment d'appartenance à un groupe. Pour le chercheur, il se doit de se faire accepter et respecter des autres à défaut de quoi, les perspectives de réussites sont considérablement diminuées.

On remarque également les problèmes de leadership formel et surtout informel lequel, s'il n'est pas bien canalisé, mine le moral de l'équipe. Il y a finalement les sentiments de frustration et de découragement qui surviennent régulièrement et qui nous portent à croire que rien n'avance.

Tous ces éléments obligent donc l'équipe à maintenir un niveau de communication très fort et à susciter des réflexions continuelles sur la démarche, le bien-fondé, les objectifs, les résultats.

Considérant ceux que nous avons déjà obtenus, tout le travail qui m'a été imposé n'a pas été vain et l'expérience acquise devient un atout pour mon cheminement futur. 


\section{3-CONCLUSION METHODOLOGIQUE}

Du point de vue théorique, méthodologique et conceptuel, ce rapport de maîtrise ne peut que confirmer la nécessité d'établir dès le départ des règles de diagnostic propices à la résolution de situations problématiques. Si la méthodologie de Checkland n'est pas la meilleure, elle est néanmoins en mesure de faire l'étude d'une situation conflictuelle comme celle de l'industrie régionale du débosselage.

Elle possède la rigidité requise pour faire avancer les travaux tout en conservant la souplesse nécessaire pour s'adapter aux changements qui se produisent régulièrement en cours de réflexion.

Elle n'est pas statique, considère l'environnement et son influence. Elle possède un langage clair, accessible à tous les intervenants dans un délai raisonnable et surtaut, elle permet d'arriver à déterminer ce qui peut être fait pour améliorer la situation. 
CONCLUSION 


\section{CHAPITRE VII}

\section{1-CONCLUSION}

La plus grande satisfaction que peut ressentir un individu, c'est de constater que les énergies qu'il a déployées dans un dossier ont pu donner des résultats probants. Celle-ci est sans doute encore plus grande lorsqu'il constate que l'abjet de son rapport de mâtrise, la mise sur pied d'un regroupement, n'est pas le compte rendu d'une suite d'évènements malheureux, mais plutôt, l'aboutissement d'une association solide, représentative et en pleine expansion.

C'est ce qui arrive dans mon cas, puisque le Regroupement des Carrossiers du Saguenay Inc existe rèellement et qu'ensemble, nous avons réussi à cheminer vers une structure qui fait l'unanimité parmi les membres. Ce qui est encore plus intéressant, c'est que le comité de direction, à chaque jour, développe de plus en plus d'autonomie à mon égard. Et, même si au fond de moi cela n'est pas sans me causer une certaine déception, je dois admettre qu'il fallait absolument en arriver à cette attitude. 
Car, nous n'avons pas élaboré un regroupement pour réaliser uniquement un rapport accadémique, mais bien pour répondre à un besoin des ateliers de débosselage du Saguenay.

I1 est sor que je continue et que je continuerai durant un certain temps encore à regarder avec attention le développement de l'association. D'ailleurs, l'exécutif m'a formulé une demande expresse en ce sens. Mais déjà, je puis dire que ceux-ci ont suffisamment progressé pour pouvoir faire cavalier seul avec un minimum d'encadrement.

L'avenir est très intéressant si l'on considère qu'à la dernière assemblée générale tenue en février 1984, on comptait pas moins de quarante personnes présentes et que le regroupement possede plus de quarante ateliers en règle. 
Cette démarche m'a finalement permis de voir la complémentarité souhaitable qui doit exister entre une institution d'enseignement telle que l'Université du Québec à Chicoutimi et le milieu régional des affaires. Celle-ci est encore plus nécessaire dans le cas des secteurs négligés par le reste des intervenants socio-économiques.

Longue vie au REGROUPEMENT DES CARROSSIERS DU

\section{SAGUENAY INC !}


ANNEXES 
ANNEXE 1

CONTRAT D'ADHESION ENTRE LE REGROUPEMENT DES CARROSSIERS

DU SAGUENAY INC ( L'ASSOCIATION

ET $\ldots \ldots \ldots \ldots \ldots \ldots \ldots \ldots \ldots \ldots$
$(L E$ PROPOSANT MEMBRE $)$

1.- Par la présente, je sousigné ..................... de l'entreprise $\ldots \ldots \ldots \ldots \ldots \ldots \ldots \ldots$ demande a.............. etre admis comme membre du Regroupement des Carrossiers du Saguenay Inc.

2.- Je déclare:

a.- oeuvrer dans le domaine de la peinture et du débosselage.

b.- opérer un commerce de peinture et de débosselage en tant que propriétaire ou son représentant doment autorisé;

c.- etre enregistré auprès du Comité Paritaire de l'Automobile, organisme responsable de l'application du décret régissant les activités du débosselage depuis le premier juin 1983; 
d.- être considéré comme étant une personne ou une entreprise responsable, effectuant un travail professionnel;

$$
\begin{aligned}
& \text { e.- répondre à toutes les exigences du décret; } \\
& \text { f.- posséder mes licences d'affaire me }
\end{aligned}
$$

permettant d'exercer mes activités.

3.- Je m'engage:

a.- à respecter tous les règlements de

l'Association ;

b.- payer à la signature des présentes la cotisation exigible et a acquitter par la suite a toutes les annés, tant que je serai membre, la cotisation annuelle alors en vigeur et ce, dans les quinzes (15) jours de la réception de l'état de compte au plus tard; c.- à respecter le code d'éthique de l'Association;

d.- a participer activement, dans toute la mesure du possible, aux activités du Regroupement;

e.- à afficher sur et dans mon établissement tout document, affiche ou autre exigés par l'Association;

f.- à faire la promotion de mon Regroupement; 
g.- à rapporter toutes situations illégales ou irrégulières au C.A.;

h.- a respecter les décisions prises majoritairement en assemblée dament convoquée à cette fin;

i.- a etre un compétiteur loyal, professionnel et respectueux;

j.- à ne pas poser d'actions, gestes qui iraient à l'encontre du bien-être de l'Association et de ses membres.

4.- Je conviens également que toute dérogation a ces conditions pourra entralner mon expulsion de' l'Association et la perte de mes droits et privilèges ainsi que toute cotisation ou partie de cotisation déja payée.

Par ailleurs, le Regroupement des Carrosiers du Saguenay Inc représenté par son président .................... s'engage par les présentes à a.- exécuter les décisions prises par l'assemblée générale; 
b.- défendre les intérêts de tous les membres sans préjugés;

c.- poser des actions prudentes dans la réalisation des objectifs approuvés par l'assemblée;

d.- utiliser avec discernement et modération

les biens, argents qui lui seront confiés par les membres;

e.- produire des rapports périodiques sur les activités;

f.- négocier auprès des fournisseurs des conditions particulières et intéressantes pour les membres;

g-- représenter adéquatement l'Association auprès de tous les corps de la société et en particulier, le comité paritaire de l'automobile et les compagnies d'assurance;

h.- aviser tous les membres de toute information pouvant modifier leurs activités;

i.- remettre à chaque membre accepté une carte de membre, une copie du code d'éthique et des règlements et des auto-collants à être apposés sur les commerces. 
En foi de quoi, le présent contrat a été signé à $\ldots \ldots \ldots \ldots \ldots \ldots \ldots$ le $\ldots \ldots \ldots \ldots \ldots$ jour de $\ldots \ldots \ldots \ldots \ldots 198 \ldots$

LE REGROUPEMENT DES CARROSSIERS DU SAGUENAY INC

PAR $: \ldots \ldots \ldots \ldots \ldots \ldots \ldots$

LE PROPOSANT MEMBRE

$\mathrm{PAR}: \ldots \ldots \ldots \ldots \ldots \ldots \ldots$

Membre acepté à la réunion du conseil d'administration $\mathrm{du}: \ldots \ldots \ldots \ldots \ldots \ldots$

Par: $\ldots \ldots \ldots \ldots \ldots \ldots \ldots \ldots \ldots \ldots \ldots$

Secrétaire 


\section{ANNEXE 3 \\ REGROUPEMENT DES CARROSSIERS DU SAGUENAY INC}

\section{CODE D'ETHIQUE}

1. Offrir au client le meilleur produit et la meilleure qualité de service.

2. Effectuer un service de haut niveau professionnel, avec probité, intégrité et compétence.

3. S'intéresser à toute forme de perfectionnement et d'amelioration de notre commerce et profession.

4. Aider notre client en étant disponible, lui offrant des solutions et lui donnant des conseils appropriés.

5. Maintenir un haut degré de coopération et de courtoisie dans nos rapports avec le client, le public, les autres membres de l'Association et les médias d'information.

6. Maintenir les locaux propres et fonctionnels. 
7. Respecter les statuts et règlements de mon Association en particulier en ce qui concerne les tarifs minimaux acceptés.

8. Tenir affichés et à vue la licence de garagiste, la carte de compétence, le code d'éthique le tarif minimum et les auto-collants de l'Association.

9. Détenir une police d'assurance responsabilité publique couvrant les dommages aux véhicules de nos clients.

10. Porter à la connaissance de l'Association toutes propositions, évènements ou autres pouvant avoir une influence sur l'ensemble des membres.

11.0ffrir une garantie à nos clients pour les travaux effectués respectant la Loi de Protection du Consommateur.

12. Donner des conseils à nos clients sur la protection des véhicules. 
Le regroupement est sans contredit la formule de l'avenir pour la petite entreprise. Certaines expériences, dans les grands centres urbains, tendent de plus en plus à le confirmer.

Cependant, pour assurer la survie d'un tel regroupement sur une base long terme, il est essentiel qu'il repose sur une structure solide et bien implantée. A cet effet, il serait inportant que vous répondiez consciencieusement, avec toute l'attention requise et en toute liberté aux questions qui suivent. Nous pouvons vous assurer que l'anonymat le plus complet sera préservé.

\section{PARTIE I}

1. Quelles sont parmi les raisons énumérées ci-après, celles qui pourraient vous inciter à vous regrouper ? Leur attribuer une cote de 1 à 8 , indiquant la plus importante à la moins importante.

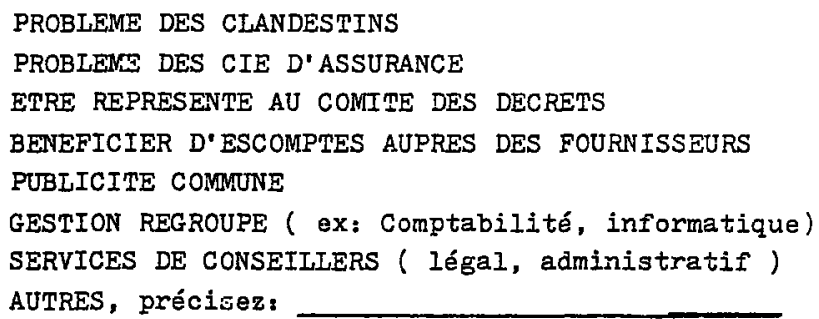

2. Combien d'employés comprend votre étabilissement par catégorie d'emplois ?
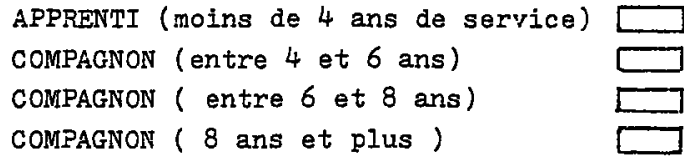

SECRETAIRE COMPIABLE HOMVE DE SERVICE

3. Existe-t-il actuellement un syndicat dans votre entreprise ?

OUI $\square$ NON

4. Comment qualifieriez-vous les relations de travail à l'intérieur de votre entreprise actuellement ?

$\begin{array}{ll}\text { TENDUES } & \square \\ \text { DIFFICILES } & \square \\ \text { ACCEPTABLES } & \square \\ \text { DETENDUES } & \square \\ \text { EXCELLENTES } & \square\end{array}$

5. Pourriez-vous indiquer brièvement, s'il y a lieu quels sont les problèmes majeurs auxquels vous faites face actuellement en tant qu'entreprise du secteur du débosselage?

\section{PARTIE II}

6. Faites-vous présentement partie d'autres associations ?

si oui lesquelles.

NON 
7. Avez-vous des ccmentaires particuliers à formuler vis à vis de ces Associations ?

8. Avez-vous des commentaires particuliers à formuler vis à vis de l'association actuellement en fornation ? Croyez-vous qu'elle soit sur la bonne voie et quelles pourraient etre les causes d'échec de l'association en formation?

9. Croyez-vous qu'il pourrait être utile que les membres puissent faire une évaluation périodique de leur association ?

OUI

NON $\square$

\section{PARTIE III}

10. Dans quelle catégorie se situe votre chiffre d'affaire ?
MOINS DE 100,000 .
100,000 . à 200,000 .
200,000. à 300,000. $\square$
300,000 . à 400,000 .
400,000 . à 500,000 .
PLUS DE 500,000 .

11. Pour chacun des produits suivants veillez indiquer:

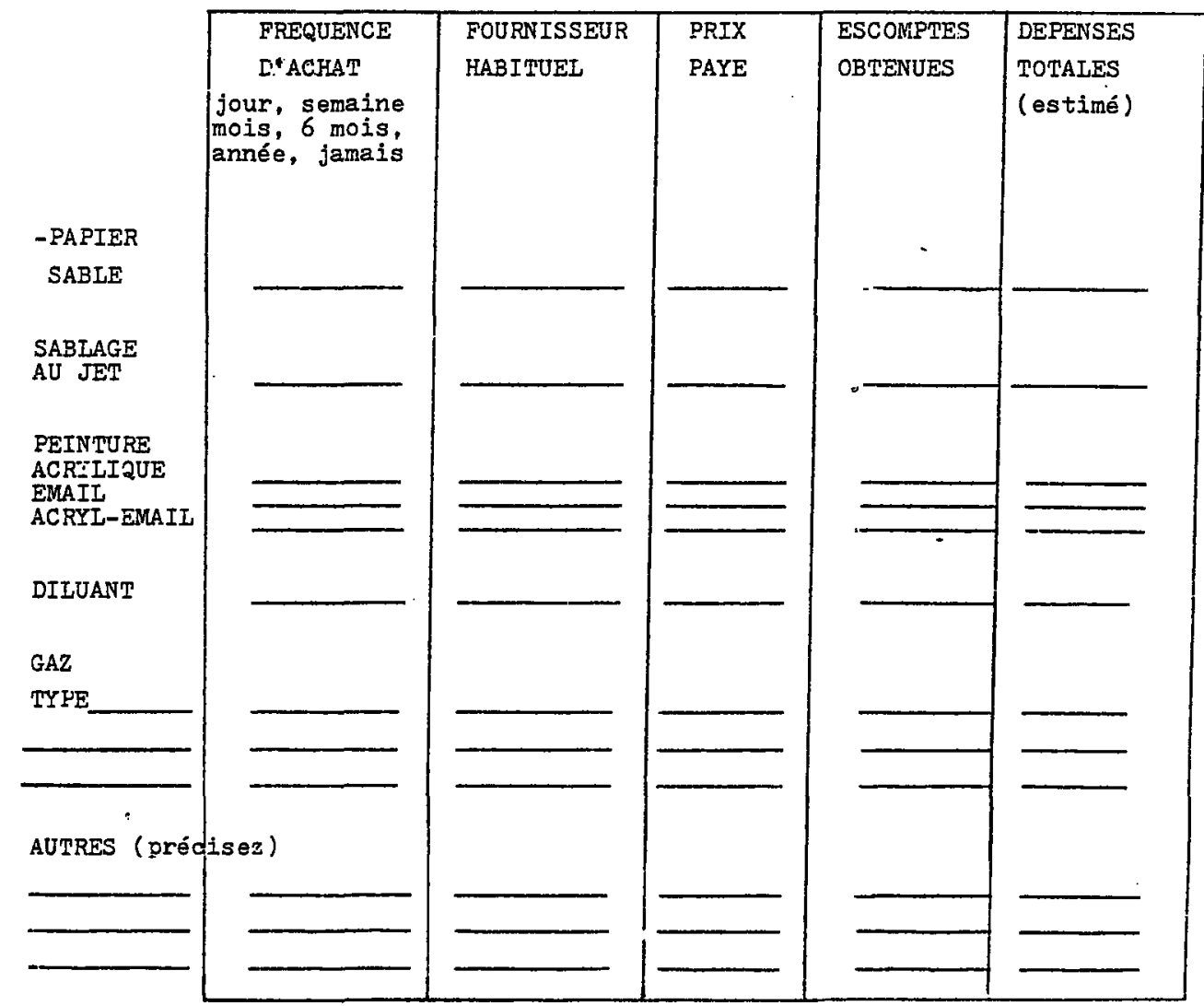


12. Croyez-vous qu'il soit possible d'obtenir de meilleurs prix par l'entremise de l'Association?

OUI $\square \quad$ NON $\square$

13. Pour chacune des compagnies suivantes veillez indiquer:

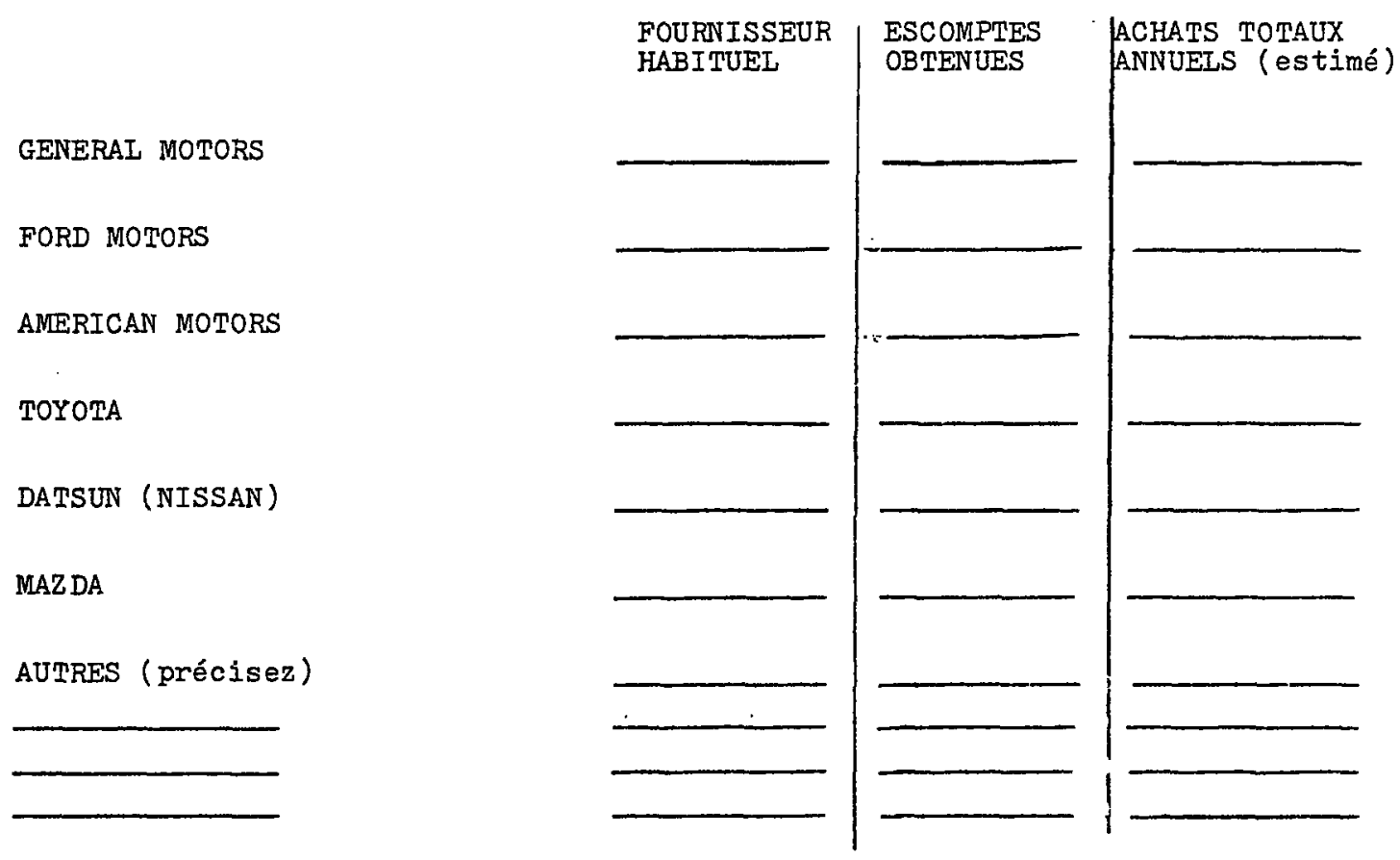

14. A votre avis, en ce qui concerne les achats de pièces et de fournitures, quels sont les problèmes majeurs que vous rencontrez. 
15. Offrez-vous un service de remorquage ?

$$
\text { OUI } \square \quad \text { NON } \square
$$

16. Faites-vous effectuer certains travaux en sous-traitance?

$$
\text { OUI } \square \text { NON } \square
$$

Si oui, les quels

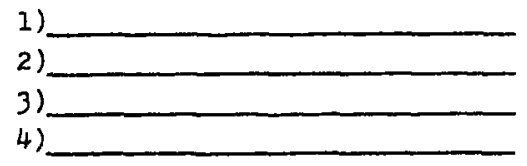

17. Pour chacun des services suivants, veillez indiquer la fréquence d'utilisation de même que le coût approximatif annuel si possible .

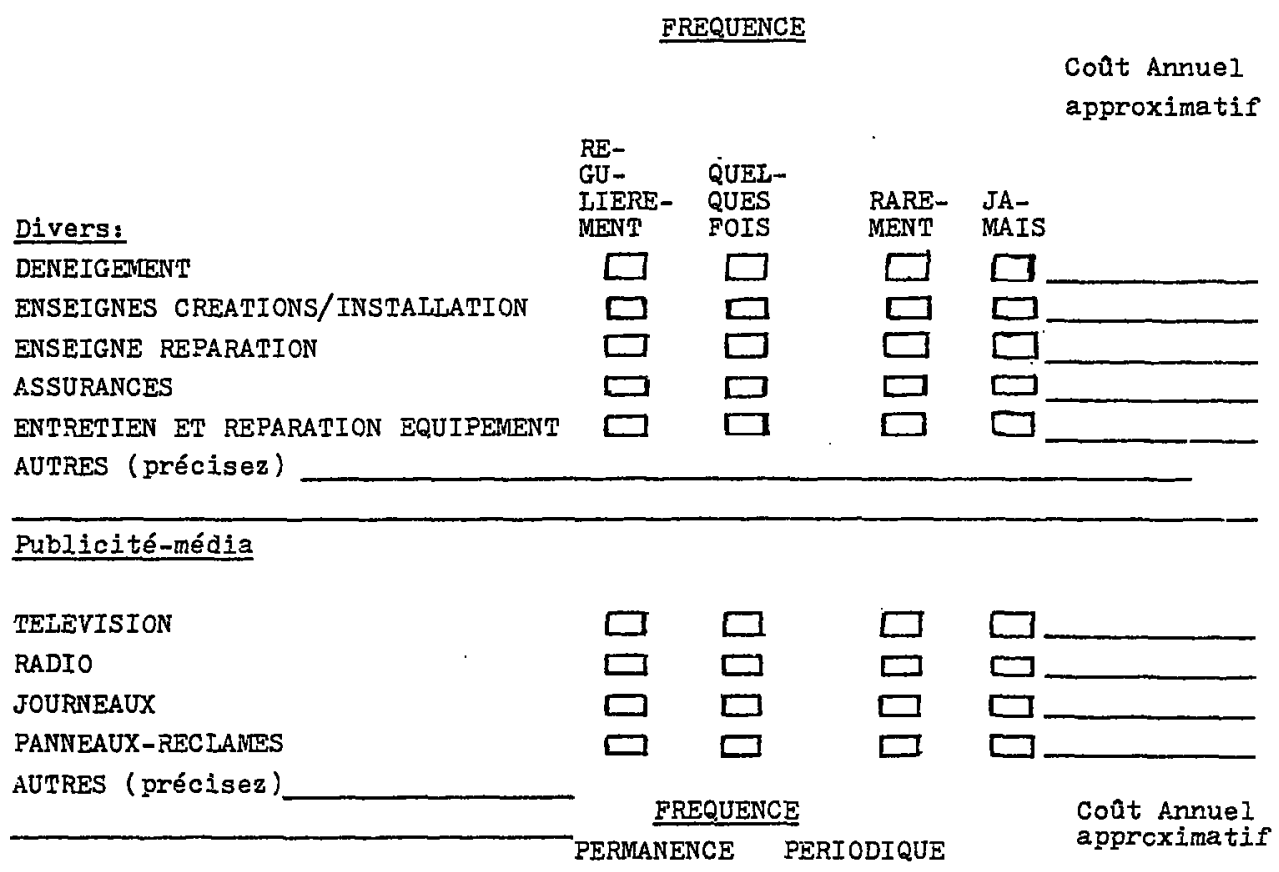

\section{Articles publicitaires personnalisés}

CARTES D'AFFAIRE

AUTO-COLIANTS

AUTTRES (précisez)

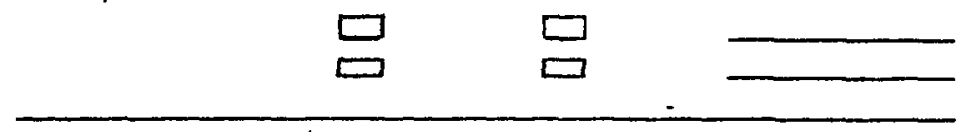

18. Pour quels articles particuliers faites-vous habituellement appel aux services d'imprimerie ? 
Dans le but de permettre à votre Association de pouvoir agir efficacement dans son travail pour éliminer les garrages clandestins, nous avons besoin de votre aide pour mettre à jour tous ces ateliers. Nous vous demandons de compléter un tel questionnaire pour chaque atelier qui selon vous effectue un tel genre de travail. Il est important que vous nous foumissiez le plus de renseignements possibles. Ceci facilitera les vérifications aux enquêteurs et nous permettra de monter un dossier complet sur la situation avec des recommandations sévères surtout pour les amendes et autres actions directes qui doivent être prises rapidement pour enrayer ces pratiques.

Nous désirons mentionner que ce formulaire sera utilisé par l'Association et les denorciations seront faites non pas par vous mais par l'Association.

PARTIE I IDENTIFICATION

I. NOM DU GARRAGE

2. ADDRESSE

3. NO DE TELEPHONE

4. NOM DU PROPRIETAIRE ( s'il vous est connu)

5. NOMBRE DE PERSONNES TRAVAILLANT LORS DE LA VERIFICATION

6. Pouvez-vous identifier certaines de ces personnes ? OUI NON

Si oui lesquelles :

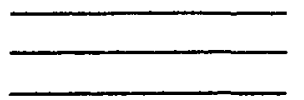

PARTIE II OPERATION DU GARRAGE

7. Avez-vous apperçu des véhicules dans le garrage ou dans la cour au moment de la vérification?

Si oui précisez
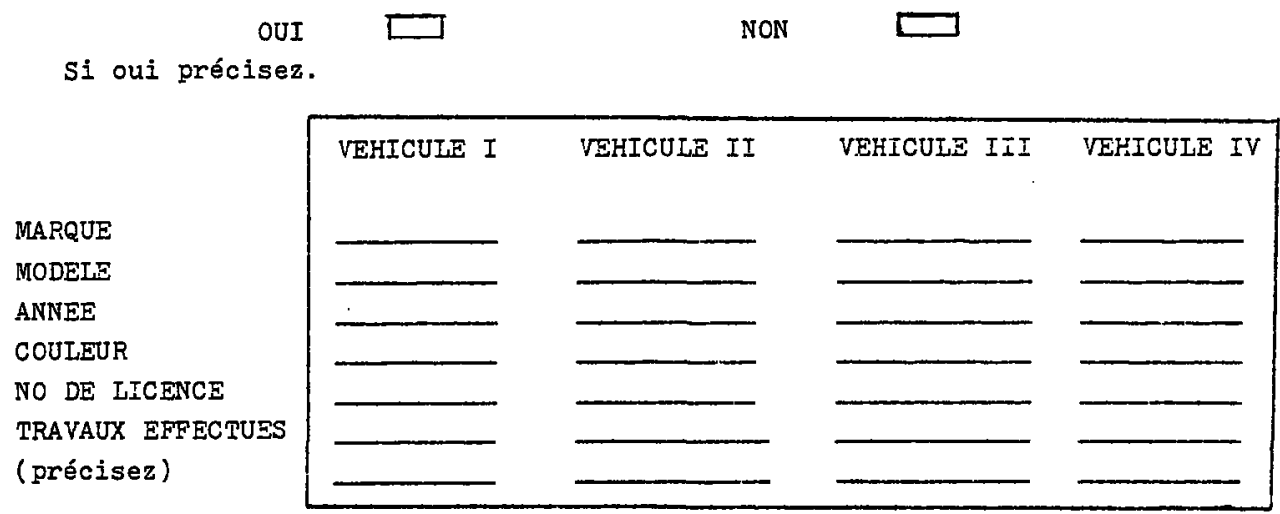

NOTE: Si l'espace est insuffisant, continuer sur une autre feuilie

PARTIE III AUTRES INFORMATIONS

8. Conditions des lieux, PROPRE $\square$ SATISFAISANT $\square$ NEGIEGE $\square$

9. Danger pour la sécurité et l'environnement oUI $\square$ NON $\square$ (exemple: matières dangereuses)

9. Avez-vous d'autres détails importants ? OUI $\square$ NON $\square$ Si oui, prócisez:

DATE DE L'IDENTIFICATION: HEURE: DERSONNE REMPIISSANT CETTE FICHE: TEMOINS

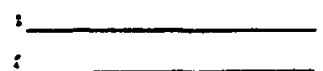


Gouvernement du Québec

L'Inspecteur général

des institutions financières
LETTRES PATENTES

Loi sur les compagnies

(L.R.Q., chap. C-38, a. 218)

Partie III

L'Inspecteur général des institutions financières, sous l'autorité de la partie 111 de la Loi sur les compagnies, accorde les présentes lettres patentes aux requérants ci-après désignés, les constituant en corporation sous la dénomination sociale

\section{LE REGROUPEMENT DES CARROSSIERS DU SAGUENAY INC.}

2

Données et scellées à Québec, le 19830609

et enregistrées le 19830622

au libro $\mathrm{C}-1142$, folio 130

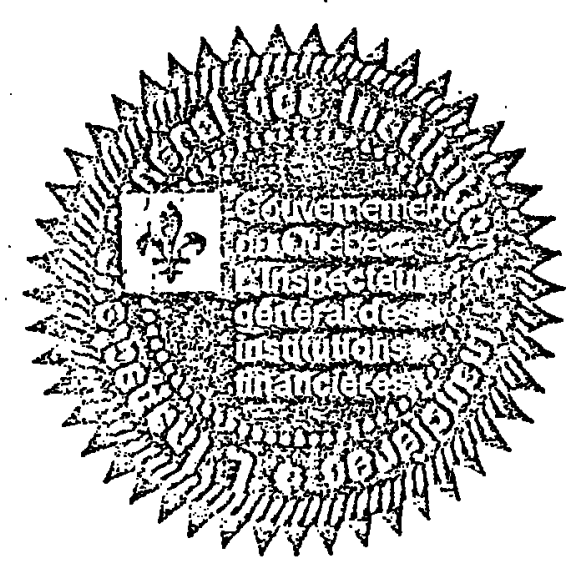




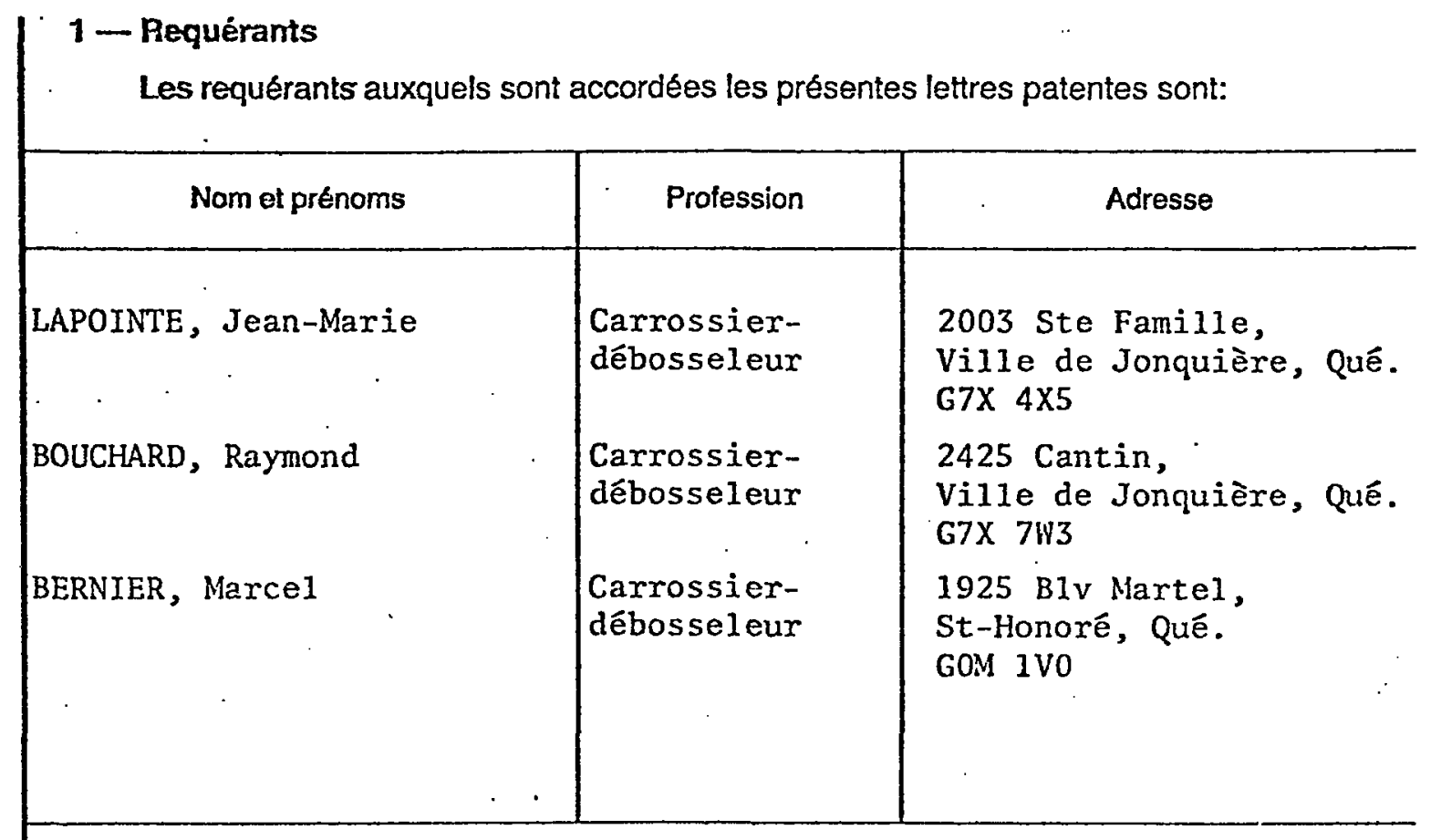

\section{2- Siège social}

Le siège social de la corporation est situé à Chicoutimi, district judiciaíre de Chicoutimi.

\section{3 - Conseil d'administration}

Les administrateurs provisoires de la corporation sont:

LAPOINTE, Jean-Marie

BOUCHARD, Raymond .

BERNIER, Marcel

\section{4-Immeubles}

La valeur des biens immobiliers que peut posséder la corporation est limitée à Cinquante mille dollars $(\$ 50,000.00)$ 


\section{5 - Objets}

Les objets pour lesquels la corporation est constituée sont les suivants:

1- Regrouper les carrossiers et/ou débosseleurs de la région du Saguenay;

2- Effectuer des études aux fins de connâ̂tre le marché du commerce de carrossier-débosselage et proposer aux membres, aux autorités gouvernementales et aux autres intervenants des suggestions utiles à la promotion de tel commerce;

3- Agir auprès des autorités gouvernementales de tous. niveaux et auprès des autres intervenants dans le commerce de carrossierdébosselage pour l'amélioration des lois et règlements ainsi que des conditions d'exercice du commerce;

4- Vaquer à procurer aux membres des informations juridiques et adminiatratives aptes à favoriser la rentabilité de leur commerce;

5- Promouvoir entre les membres une concurrence honnête et susceptible d'améliorer les conditions du marché;

6- Faire des sessions d'étude et d'information et effectuer auprès des membres la publication de toute littérature permettant d'atteindre les buts visés;

7- Informer le public en général sur les problènes et les préoccupations des carrossiers-débosseleurs et sur les services qu'ils offrent;

8- Procurer à ses membres des activités sociales utiles à la réalisation de ses buts en resserrant entre eux les liens d'amitié et de fraternité; 
Cabinel du ministre du Travail 150. boul. St-Cyrille est 8e étage

Québec

G1R2B2

Québec, le 29 juillet 1983

Monsieur Hubert Desbiens

Député de Dubuc

1226, avenue du Port

Ville de la Baie

G7B $1 W 4$

Monsieur le député,

La présente a pour but de donner suite à ma précédente lettre du 15 juillet 1983 et la vôtre du 12 juillet 1983.

Concernant les pratiques utilisées par les compagnies d'assurances de négocier à la baisse les tarifs en vigueur, vous comprendrez qu'il n'appartient pas au ministre du Travail d'intervenix ou de légiférer en cette matière.

Quant à l'existence. de garages clandestins, il est évident que l'actuelle Loi sur les décrets de convention collective, ne contribue pas, par la légèreté de ses amendes, à restreindre ce fléau.

Cette lacune devrait être comblée par le dépôt d'un projet de loi modifiant la loi sur les décrets de convention collective cet automne.

Enfin, sur le point de la représentation, les débosseleurs du Saguenay - Iac st-Jean auraient tout intérêt à se constituer en association, et ce dans le but de faire connaitre leurs revendications au comité paritaire et lors de modifications au décret.

En espérant que ces quelques informations vous seront utiles, je vous prie d'agréer, monsieur le député, $l$ 'expression de mes sentiments distingués.

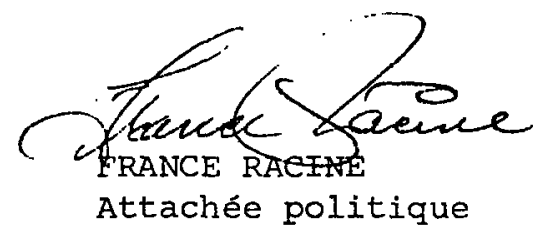




\section{BIBLIOGRAPHIE}

CHECKLAND, P.B., Systems Theory, Systems Practice, Wiley, 1981 .

FORTIN; Gérald, De quelques significations de la recherche-action, Actes du colloque Recherche-action, UQAC, Chicoutimi,1981, p.7.

PREVOST, Paul, Le diaqnostic-intervention: une approche systémique au diagnostic organisationnel et à la recherche-action, LEER, Chicoutimi, $92 \mathrm{p}$.

Roy, R., ROY, M., PREVOST, Paul et all, Vers une définition du concept de recherche-action, Revue PMD, Vol. I, No. l, Chicoutimi, 1982.

RAPPORT D'ACTIVITE 1982-1983, Régie de I'Assurance Automobile du Québec, Editeur officiel du Québec, Québec, 1983, 77 p.

RAPPORT DU SURINTENDANT DES ASSURANCES, La tarification en assurance automobile au Québec 1982, Ministère des Institutions financières et coopératives, Québec, 1983, $135 \mathrm{p}$. 